\title{
Gli uccelli del Parco Nazionale della Val Grande e delle aree limitrofe (Verbano Cusio Ossola, Piemonte, Italia)
}

\author{
Fabio Casale ${ }^{1 *}$, Cristina Movalli ${ }^{2}$, Radames Bionda ${ }^{3}$, Lorenzo Laddaga ${ }^{4}$, \\ Andrea Mosini ${ }^{5} \&$ Manuel Piana ${ }^{6}$
}

Riassunto: Il Parco Nazionale della Val Grande è un'area montuosa protetta sita nelle Alpi, nel Piemonte nord-orientale, Italia. L'area è stata designata quale Parco Nazionale nonché ZPS - Zona di Protezione Speciale secondo la Direttiva "Uccelli" e IBA - Important Bird Area secondo BirdLife International. L'area di studio ha una superficie di 45.873 ha che comprende il Parco Nazionale e le limitrofe Valle Intrasca, Valle Cannobina e Val Loana, i cui ambienti principali sono costituiti da vaste foreste di latifoglie e di conifere, ambienti rocciosi, brughiere, arbusteti e praterie alpine. Le cime più alte raggiungono i $2.300 \mathrm{~m}$ s.l.m. La maggior parte della sua superficie è selvaggia e disabitata. In questo lavoro viene riportato un elenco commentato degli uccelli noti per l'area e vengono inoltre forniti dati di sintesi relativi alle attività di monitoraggio condotte in anni recenti (2009-2014). I dati sono stati raccolti sia attraverso revisione bibliografica sia in forma originale, attraverso attività di ricerca sul campo. Queste ultime sono state condotte utilizzando i seguenti metodi: mappaggio dei territori (uccelli nidificanti degli ambienti aperti), punti d'ascolto (uccelli nidificanti), transetti lineari con uso di playback (rapaci notturni, Caprimulgus europaeus, Dryocopus martius), ricerca di campo non standardizzata in ambienti idonei alla nidificazione (Passeriformi nidificanti degli ambienti rocciosi), censimento primaverile di rapaci migratori lungo la rotta migratoria del Lago Maggiore e censimento autunnale di Passeri-

${ }^{1}$ Fondazione Lombardia per l'Ambiente, Largo 10 luglio 1976 1, 20822 Seveso (MB), Italia.

E-mail: fabio.casale@flanet.org

${ }^{2}$ Ente Parco Nazionale della Val Grande, Villa Biraghi, Piazza Pretorio 6, 28805 Vogogna (VB), Italia.

E-mail: cristina.movalli@parcovalgrande.it

${ }^{3}$ Società di Scienze Naturali del Verbano Cusio Ossola, Museo di Scienze Naturali, Collegio Mellerio Rosmini, Via Antonio Rosmini 24, 28845 Domodossola (VB), Italia.

E-mail: rada.bionda@libero.it

${ }^{4}$ Via Crotto Roncaccio 6, 21049 Tradate (VA), Italia.

E-mail: 1.laddaga@libero.it

${ }^{5}$ Valgrande Società Cooperativa, Via alla Cartiera 41, 28923

Verbania-Possaccio (VB), Italia.

E-mail: mosiniandrea@gmail.com

${ }^{6}$ Via Vignale 16, 28887 Omegna (VB), Italia.

E-mail: piana.manuel@gmail.com

*Corresponding author: fabio.casale@flanet.org

(C) 2013 Fabio Casale, Cristina Movalli, Radames Bionda, Lorenzo Laddaga, Andrea Mosini \& Manuel Piana

Received: 25 July 2014.

Accepted for publication: 18 November 2014. formi migratori attraverso i passi alpini. L'area ospita o ha ospitato 138 specie di Uccelli delle quali 97 nidificanti, 28 di interesse comunitario (Allegato I della Direttiva "Uccelli"), 42 SPEC - Species of European Conservation Concern secondo BirdLife International. L'area è di grande importanza per numerose specie di uccelli, specialmente per la nidificazione di specie di interesse conservazionistico legate ad habitat montani, quali Bonasa bonasia (20-50 coppie), Tetrao tetrix (40-100 maschi adulti), Alectoris graeca saxatilis (20-50 coppie), Circaetus gallicus (4-5 coppie), Aquila chrysaetos (6-7 coppie), Falco peregrinus (4-5 coppie), Bubo bubo (4 coppie), Aegolius funereus, Dryocopus martius (30 coppie), Monticola saxatilis (5-10 coppie), Tichodroma muraria (5-10 coppie), Pyrrhocorax graculus (50-70 coppie), Prunella collaris (10-20 coppie), Emberiza cia (200-300 coppie), per la migrazione primaverile dei rapaci diurni ( 15 specie rilevate, delle quali $10 \mathrm{di}$ interesse comunitario), soprattutto Pernis apivorus (21,3\% degli individui censiti), Falco tinnunculus (16,7\%) e Buteo buteo (14,5\%) e per la migrazione autunnale dei Passeriformi (30 specie rilevate), soprattutto fringillidi (46,9\% degli individui censiti), irundinidi $(32,8 \%)$ e motacillidi (15,6\%). Gypaetus barbatus e Gyps fulvus vi vengono occasionalmente rilevati.

Parole chiave: Alpi, conservazione, migrazione, monitoraggio, uccelli montani, Parco Nazionale della Val Grande.

Abstract: Birds of Val Grande National Park and surrounding areas (Verbano Cusio Ossola, Piedmont, Italy)

Val Grande National Park is a mountain protected area located in the Italian Alps, north-eastern Piedmont. The area was designated as National Park, SPA - Special Protection Area according to the Birds Directive and IBA - Important Bird Area according to BirdLife International. The study area has a surface of 45.873 ha and include the Park and the bordering valleys Valle Intrasca, Val Cannobina, Val Loana. Main habitats include broadleaved (mainly beech) and coniferous forests, rocky habitats, alpine heathlands and alpine grasslands. Highest peaks reach 2.300 meters of altitude. Most of its surface is not inhabited. The paper reports a description of all the bird species recorded from the area and the results of recent monitoring activities (2009-2014). Data were obtained from a large bibliographic research and from field activities. The last ones were conducted through the following methods: territory mapping (breeding birds of open habitats), point counts (breeding birds), linear transects with use of playback (owls, Caprimulgus europaeus, Dryocopus martius), non standardized field researches in suitable breeding habitats (breeding Passerines of rocky habitats), spring censuses of migratory raptors along the flyway of lake Maggiore, autumn censuses of migratory Passerines through mountain passes. We obtained evidence for the occurrence of 138 bird species: 97 of them breed in the area, 28 are of community interest (Annex I of Birds Directive), 42 are SPEC Species of European Conservation Concern according to BirdLife International. The area is of high importance for many bird species, especially for breeding mountain species of conservation interest, such 
as Bonasa bonasia (20-50 pairs), Tetrao tetrix (40-100 adult males), Alectoris graeca saxatilis (20-50 pairs), Circaetus gallicus (4-5 pairs), Aquila chrysaetos (6-7 pairs), Falco peregrinus (4-5 pairs), Bubo bubo (4 pairs), Aegolius funereus, Dryocopus martius (30 pairs), Monticola saxatilis (5-10 pairs), Tichodroma muraria (5-10 pairs), Pyrrhocorax graculus (50-70 pairs), Prunella collaris (10-20 pairs), Emberiza cia (200-300 pairs), for spring migration of raptors (15 species recorded, 10 of community interest), mainly Pernis apivorus (21,3\% of censused individuals), Falco tinnunculus (16,7\%) and Buteo buteo (14,5\%) and for autumn migration of Passerines across mountain passes (30 species recorded), mainly Fringillidae (46,9\% of the censused individuals), Hirundinidae $(32,8 \%)$ and Motacillidae (15,6\%). Gypaetus barbatus and Gyps fulvus are occasionally recorded.

Key words: Alps, conservation, migration, monitoring, mountain birds, Val Grande National Park.

\section{INTRODUZIONE}

La Val Grande è un'area di importanza internazionale per l'avifauna, tanto da essere stata designata come IBA - Important Bird Area da Bird Life International e come ZPS - Zona di Protezione Speciale secondo la Direttiva Uccelli 2009/147/CE dalla Commissione Europea. Il Parco Nazionale è stato istituito nel 1992 e a oltre vent'anni dalla sua istituzione, soprattutto a seguito di recenti attività di monitoraggio ornitologico condotte con continuità nel periodo 2009-2014, si è ritenuto opportuno proporre per la prima volta lo stato delle conoscenze dell'avifauna dell'area protetta e delle aree limitrofe, anche allo scopo di fornire una base di confronto per future attività di ricerca e monitoraggio ornitologico.

\section{MATERIALI E METODI}

Il Parco Nazionale della Val Grande è localizzato nel settore meridionale delle Alpi Lepontine, nel Piemonte settentrionale, in particolare nella provincia del Verbano Cusio Ossola. Si tratta di un complesso di rilievi montuosi caratterizzati da una morfologia aspra che racchiude i bacini idrografici del Rio Valgrande e del Rio Pogallo. Le cime più alte raggiungono i $2.300 \mathrm{~m}$ s.l.m. Lo stato di conservazione degli ambienti naturali risulta generalmente buono, con l'eccezione delle cenosi erbose al di sotto del limite della vegetazione arboreo-arbustiva, soggette a rapida "chiusura" a seguito dell'abbandono delle attività agricole tradizionali, con la conseguente sparizione di habitat di grande importanza per numerose specie vegetali (ad es. Tulipa australis e numerose specie di orchidee) e animali (ad es. numerose specie di uccelli) di interesse conservazionistico e legate agli ambienti aperti. Il paesaggio della Val Grande risente infatti della condizione di abbandono delle attività agro-silvo-pastorali, in particolare di quelle pascolive, in corso da 40-50 anni. Tale abbandono ha portato le aree precedentemente occupate da ambienti aperti (soprattutto prati da fieno e pascoli) a essere colonizzate naturalmente dalla vegetazione arboreo-arbustiva, tanto che attualmente più di tre quarti del territorio dell'area protetta è occupato da un fitto manto boschivo, composto in prevalenza da latifoglie, soprattutto faggete e castagneti (Sindaco et al., 2009).

L'area di studio comprende (Fig. 1) il Parco Nazionale e alcune aree limitrofe che presentano caratteristiche ambientali analoghe:
- la Valle Intrasca, localizzata a est del Parco Nazionale;

- ampi settori dell'Alto Verbano, a est della Valle Intrasca;

- la Valle Cannobina, a nord-est;

- la Val Loana e la Valle del Basso, a nord-ovest;

- i versanti che dalla Val d'Ossola salgono verso i confini del Parco, lungo i lati ovest e sud del Parco;

- la dorsale che porta dal Pizzo Faiè al Monterosso, a sud-est del Parco, che costituirà un probabile ampliamento dell'area protetta.

La superficie complessiva dell'area di studio è di 45.873 ha, dei quali 15.689 (il 34\%) compresi nel Parco Nazionale. Tra gli ambienti più rappresentativi si segnalano vasti boschi di latifoglie e di conifere, habitat rocciosi, arbusteti e praterie alpine (Figg. 2-6).

Il presente lavoro riassume i risultati di oltre 20 anni di osservazioni relative all'avifauna effettuate nel Parco Nazionale della Val Grande e aree limitrofe, più precisamente nel periodo 1991-2014. Il 1991, nell'ambito della Legge Quadro sulle aree protette, è stato infatti l'anno di designazione dell'area a Parco Nazionale, poi istituito con D.M. del 2 Marzo 1992. A tali osservazioni sono state altresì aggiunte segnalazioni relative al periodo precedente l'istituzione dell'area protetta, prevalentemente pubblicate in Bionda \& Bordignon (2006), Casale \& Toninelli (2005), EPNVG (1998) e Fonio (1995).

Le principali ricerche ornitologiche condotte nell'area protetta sono di seguito elencate:

- 1993 - Studio sulla comunità ornitica tramite punti di ascolto (Movalli \& Grimaldi, 1996);

- 1997 - Studi faunistici per il Piano del Parco (EPNVG, 1998);

- 2009 - Studio sull'avifauna degli ambienti aperti (Casale \& Brambilla, 2010b);

- 2010 - Analisi dei dati relativi alle specie di interesse comunitario per il Piano di Gestione della ZPS "Val Grande" (Casale \& Brambilla, 2010a);

- 2013-14 - Monitoraggio di picchio nero, succiacapre, rapaci notturni, avifauna nidificante negli ambienti rupestri, migrazione primaverile dei rapaci diurni, migrazione autunnale dei Passeriformi (Casale, 2014);

- 2013-14 - Monitoraggio della biodiversità alpina in plot distribuiti lungo transetti: per l'avifauna nidificante sono stati eseguiti punti d'ascolto due volte all'anno in periodo riproduttivo, a giugno e a luglio (Casale, 2014).

Per la nomenclatura e l'ordine sistematico ci si è riferiti alla Lista CISO - COI degli Uccelli italiani (Fracasso et al., 2009).

Le foto aeree utilizzate sono tratte dal sito web "Immagini TerraItaly ${ }^{\mathrm{TM}}$ - CBlom CGR S.p.A. - Parma www.terraitaly. it". Si è provveduto al loro utilizzo a seguito di autorizzazione pervenuta da Blom CGR S.p.A. all'Ente Parco Nazionale della Val Grande con lettera del 26 marzo 2010.

\section{ELENCO SISTEMATICO DELLE SPECIE RILEVATE}

Di seguito viene fornito un elenco commentato delle specie di Uccelli del Parco Nazionale della Val Grande e 
aree limitrofe. Le categorie fenologiche utilizzate sono le seguenti:

\section{S: Sedentary - Sedentaria}

B: Breeding-Nidificante (se con punto di domanda significa che la nidificazione è solo probabile e da accertare)

M: Migratory - Migratrice (in questa categoria sono incluse anche le specie dispersive e quelle che compiono erratismi di una certa portata)

W: Wintering - Svernante (in questa categoria sono incluse anche specie la cui presenza nel periodo invernale non sembra assimilabile a un vero e proprio svernamento) A: Accidental-Accidentale

Ext: Extinct - Estinta

irr: irregular - irregolare

reg: regular - regolare

I nomi degli autori di gran parte delle osservazioni sono così abbreviati: Radames Bionda (RB), Michele Bove (MB), Fabio Canepuccia (FCA), Fabio Casale (FC), Fabrizio Clemente (FCL), Pier Francesco Donzelli (PD), Marco Dresco (MD), Diego Fontaneto (DF), Aldo Genoni (AG), Lorenzo Laddaga (LL), Andrea Mosini (AM), Cristina Movalli (CM), Manuel Piana (MP), Pietro Pisano (PP), Lucia Pompilio (LP), Aldo
Poppi (AP), Diego Ramoni (DR), Simone Torniai (ST). Infine, con BDVG si intende la Banca Dati Faunistica del Parco Nazionale della Val Grande, realizzata in collaborazione con il Corpo Forestale dello Stato (CFS).

\section{ANSERIFORMES}

\section{Anatidae}

Germano reale (Anas platyrhynchos) $\mathrm{M}$

La specie viene raramente osservata lungo i principali corsi d'acqua che interessano l'area di studio, i torrenti San Bernardino e San Giovanni (ad esempio un maschio il 18/05/2000 sul torrente San Giovanni, nel tratto compreso tra Intragna e Scareno: FC) e il Rio Cannobino. Si tratta verosimilmente di individui in erratismo, provenienti dalla limitrofa Riserva Naturale di Fondotoce e dalle vicine sponde del Lago Maggiore, dove la specie è comunemente nidificante.

\section{Smergo maggiore (Mergus merganser) $\mathrm{M}$}

Poche segnalazioni per questa specie che nidifica regolarmente in aree limitrofe all'area di studio, lungo le rive del Lago Maggiore e lungo il tratto planiziale del fiume

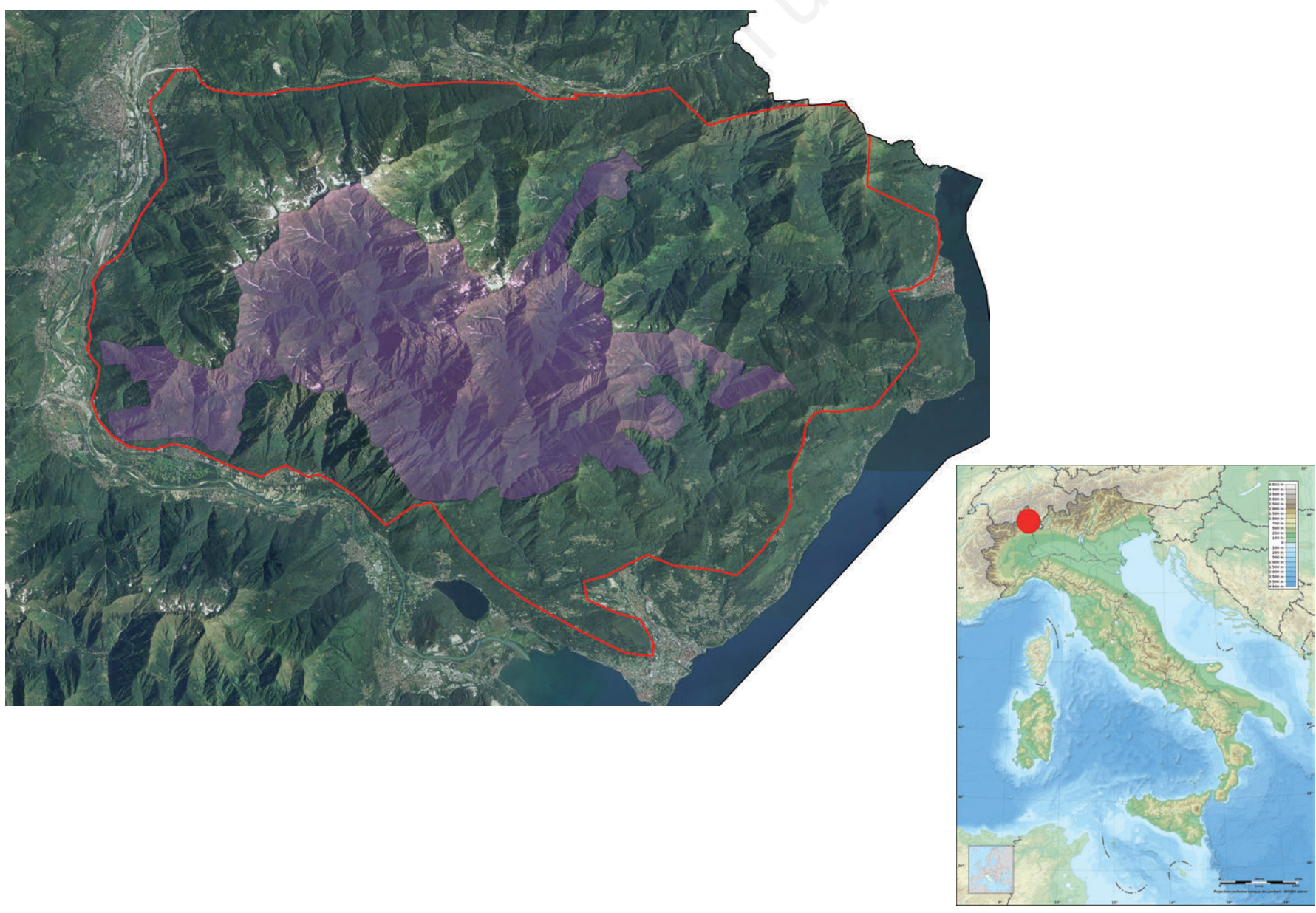

Fig. 1 - Localizzazione dell'area di studio (in rosso i confini) e del Parco Nazionale della Val Grande (superficie violetto). A W e a SW è visibile il fiume Toce e a E e SE il Lago Maggiore. / Location of the study area (borders in red) and of Val Grande National Park (surface in purple). On the W and SW sides of the area is visible the Toce river and on the E and SE sides the Lake Maggiore. 
Toce. Una coppia è stata osservata in Val Vigezzo lungo il torrente Melezzo orientale, presso Villette, il 29/01/2014 (LP), ed una lungo il torrente Melezzo occidentale a inizio maggio 2014 (DR); 7 individui in volo sopra il torrente Cannobino all'altezza di Spoccia il 14/08/2014 (FC, MB).

\section{GALLIFORMES}

\section{Phasianidae}

\section{Francolino di monte (Bonasa bonasia) SB}

In Piemonte la specie si sarebbe estinta nel XIX secolo. L'ultima cattura avvenne in Val d'Ossola nel 1825 e né Bazzetta (1893) né Dell'Angelo (1887) ritenevano possibile che fosse presente alla fine dell'Ottocento. La ricolonizzazione della Val d'Ossola risale all'inizio degli anni '40 del XX secolo e durante l'indagine condotta negli anni 1980-1984 la specie era ben distribuita in Val d'Ossola (Mingozzi et al., 1988). Nel settore ossolano dell'area di studio, esterno ai confini del Parco (comuni di Beura-Cardezza e Trontano) il francolino di monte (Fig. 7) era ben presente e nidificante negli anni '70 e '80 del XX secolo, con territori occupati a partire da circa $1000 \mathrm{~m}$ di quota. Negli anni '90 del XX secolo è stata osservata una contrazione della fascia altimetrica utilizzata, con la progressiva scomparsa dei territori posti al di sotto dei 1200-1300 m s.l.m., verosimilmente legata alle trasformazioni del paesaggio avvenute in questa fascia altitudinale. La presenza della specie all'interno del Parco nel corso del XX secolo era indicata come "sporadica" nel Piano del Parco (EPNVG, 1998), valutando la struttura vegetazionale dell'area protetta inadatta ad ospitare una popolazione vitale di francolino di monte, in assenza di formazioni forestali climaciche con un sottobosco sviluppato. D'altro canto, gli estensori dello studio ritenevano che la specie potesse tornare a ricolonizzare spontaneamente l'area protetta a seguito dell'evolversi della vegetazione naturale verso condizioni ottimali per la specie. Ė infatti verosimile che la successiva evoluzione spontanea della vegetazione forestale abbia favorito la formazione di habitat maggiormente idonei a ospitare il francolino di monte in quanto negli ultimi 10 anni la specie ha avviato un processo di colonizzazione che l'ha portata ad essere segnalata con sempre maggiore regolarità (Casale \& Brambilla, 2010a). Benché non sia stata oggetto di specifici censimenti nell'area protetta, la raccolta di dati da parte del Corpo Forestale dello Stato, coordinata dall'Ente Parco, ha infatti permesso negli ultimi anni di documentare la presenza sempre più regolare della specie in alcuni settori e nel 2009 è stata accertata per la prima volta la sua nidificazione nel Parco sulla dorsale del Pizzo Mottac, con l'osservazione di un adulto con un giovane il 19/07/2009 e, nella stessa data, di un adulto con 5 giovani al Fornale di Rina (BDVG). Negli anni successivi si segnalano le seguenti nidificazioni accertate:

- femmina con 3 nidiacei sulla dorsale del Pizzo Mottac il 22/07/2010 (BDVG);

- femmina con 5 giovani sotto la Cappella di Terza il 30/08/2013 (AM);
- femmina con covata poco sotto la Bocchetta di Terza il 3/08/2014 (AM);

- femmina con 2 giovani tra Scaredi e La Balma l'11/09/2014 (AM).

La sua presenza nell'area di studio è stata altresì

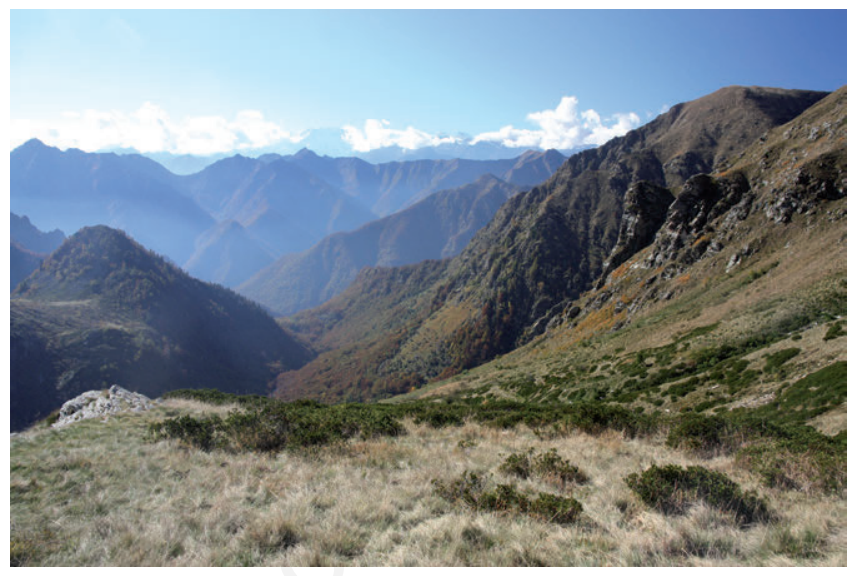

Fig. 2 - La Val Grande da Scaredi. / Val Grande from Scaredi. (Foto / Photo Fabio Casale).

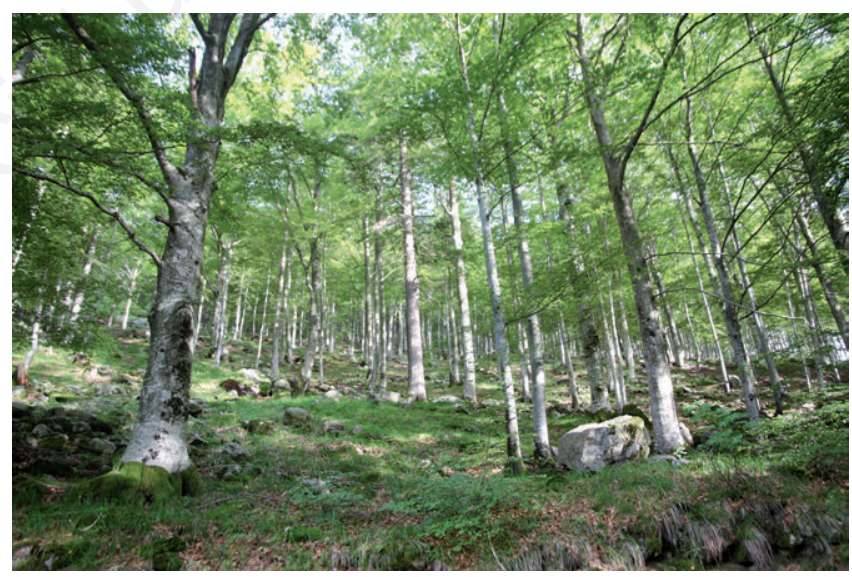

Fig. 3 - Faggeta. / Beech forest. (Foto / Photo Fabio Casale).

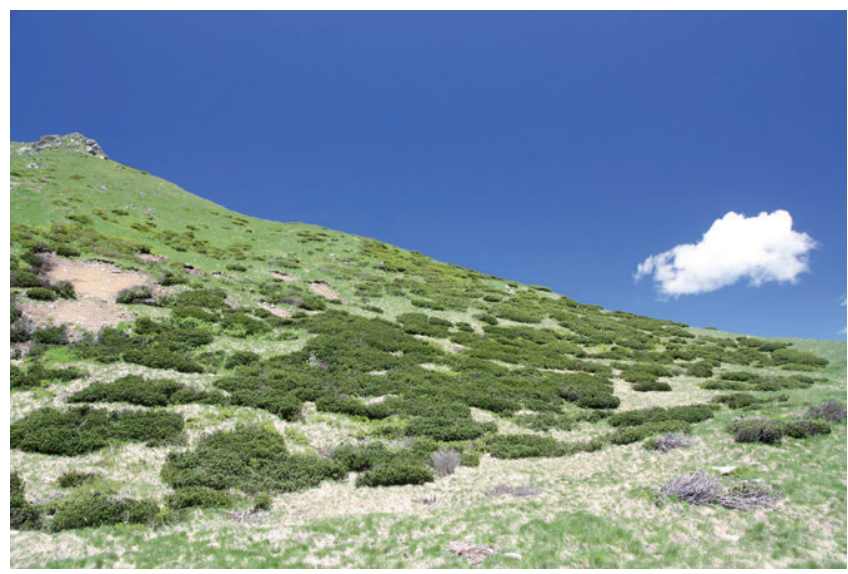

Fig. 4 - Rodoreto alle pendici del Pizzo Stagno. / Alpine heathlands at Pizzo Stagno feet. (Foto / Photo Fabio Casale). 
rilevata dal personale del CFS in corrispondenza delle seguenti località: Fondo Li Gabbi (18/10/2000), Val Piana (22/08/2004), Vald (28/08/2006), tra Bocchetta di Campo e Baita Seriago (09/11/2006), Miucca (29/09/2007), Pizzo Pernice (23/10/2009 e 26/12/2009), Forcola-Onunchio (12/11/2010), Alpe Serena (7/11/2012), Alpe Boschelli (27/09/2013), Alpe Cavalla (17/07/2014, LL).

E verosimile stimare che nell'area di studio siano presenti 20-50 coppie.

Pernice bianca (Lagopus muta) Ext

Nel Parco la specie era ben rappresentata negli anni '50 del XX secolo sulla Cima della Laurasca, al confine settentrionale dell'area protetta, e nella prima metà degli anni '80 nidificava ancora sul Monte Togano (Bionda \& Bordignon, 2006). Dati di presenza nel settore settentrionale del Parco (aree tra Bocchetta di Campo e Cima della Laurasca e tra Cimone di Straolgio e Pizzo Stagno) con esemplari abbattuti fino al 1995 durante la caccia vengono riferiti in EPNVG (1998), sulla base di informazioni raccolte da interviste. È probabile che su queste popolazioni, poste ai margini dell'area di distribuzione, abbia avuto un impatto fortemente negativo anche un'eccessiva pressione venatoria (Bionda \& Bordignon, 2006). La specie non è stata rilevata nel Parco nel periodo 2000-2014 e deve ritenersi estinta, almeno come nidificante.

\section{Fagiano di monte (Tetrao tetrix tetrix) SB}

Negli studi preliminari alla stesura del Piano del Parco (EPNVG, 1998) la specie veniva valutata come diffusa negli ambienti idonei dell'area protetta, seppure con densità basse. La quasi totalità delle osservazioni raccolte tra il 1990 e il 1997 e citate nel Piano si concentrava nel settore settentrionale del Parco (Straolgio, Scaredi, Alpe Campo, Alpe e dorsale del Mottac per il settore nordoccidentale, Alpe Busarasca e Alpe Terza per quello nordorientale). In questi studi non era segnalata la presenza di arene con un numero di maschi superiore a 2-4 individui (ad esempio, nel 1994 un'arena ubicata presso l'Alpe Vald di Sopra con 4 individui in canto; CM). Rilievi svolti nell'area di Scaredi avevano portato a stimare densità

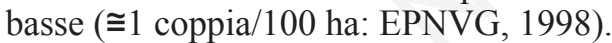

Nel periodo 2000-2014 i dati raccolti dal personale del CFS (oltre 100 rilevamenti occasionali) hanno confermato i siti di presenza nel settore settentrionale e hanno aggiunto nuovi siti, ad esempio verso le propaggini più meridionali del Parco, anche ad altitudini medio-basse per le abitudini della specie. Tra le nuove aree in cui è stata rilevata la presenza si segnalano:

- settore sud-occidentale: alpeggi lungo entrambi i versanti della Colma di Premosello (Fig. 8), Corni di Nibbio, valle dei laghetti del Proman, Cima Corte Lorenzo e Corte Bué;

- settore sud-orientale: Colma di Belmello, dorsale del Pizzo Pernice fino al Pian Cavallone e al Colle della Forcola, dorsale dall'Alpe Archìa al Monte Zeda (Pian Puzzo-Pian Vadà-Pian d'Arla);

- aree confinanti con il Parco, nei Comuni di BeuraCardezza e Trontano a ovest (Alpe Cortevecchio, Alpe Rina), a nord in Valle del Basso (Alpe
Bondolo) e in Val Loana (Alpe Cortenuovo, Alpe Cortevecchio).

Nel 2009 l'Ente Parco ha svolto per la prima volta un censimento sperimentale della specie nel territorio dell'area protetta (De Franceschi, 2009) utilizzando

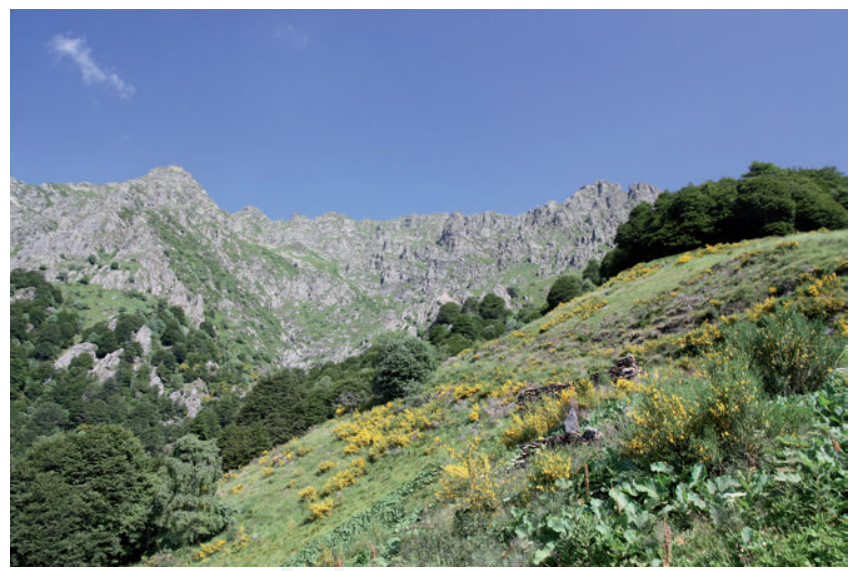

Fig. 5 - Pascoli abbandonati e ambienti rocciosi in alta Val Pogallo. / Abandoned pastures and rocky habitats in upper Val Pogallo. (Foto / Photo Fabio Casale).

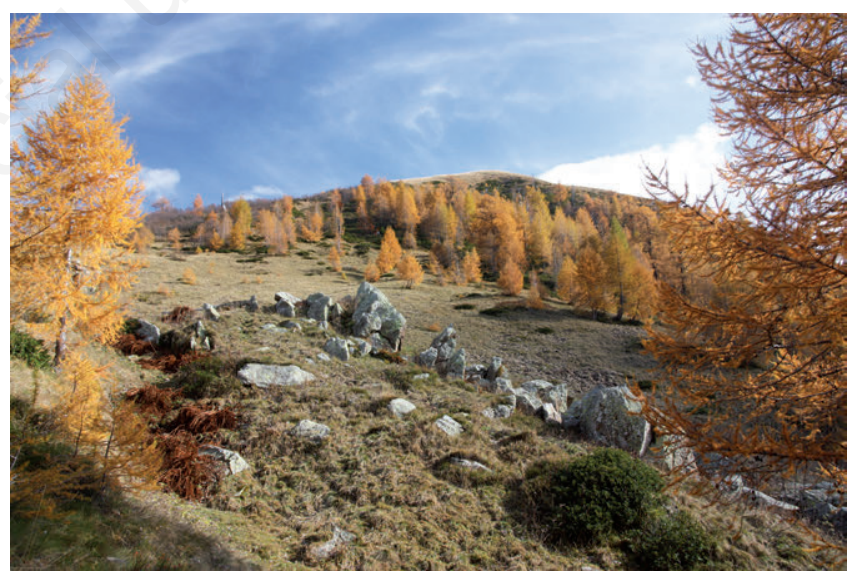

Fig. 6 - Lariceta a parco nei pressi della Bocchetta di Cavalla. / Open larch forest close to Bocchetta di Cavalla. (Foto / Photo Fabio Casale).

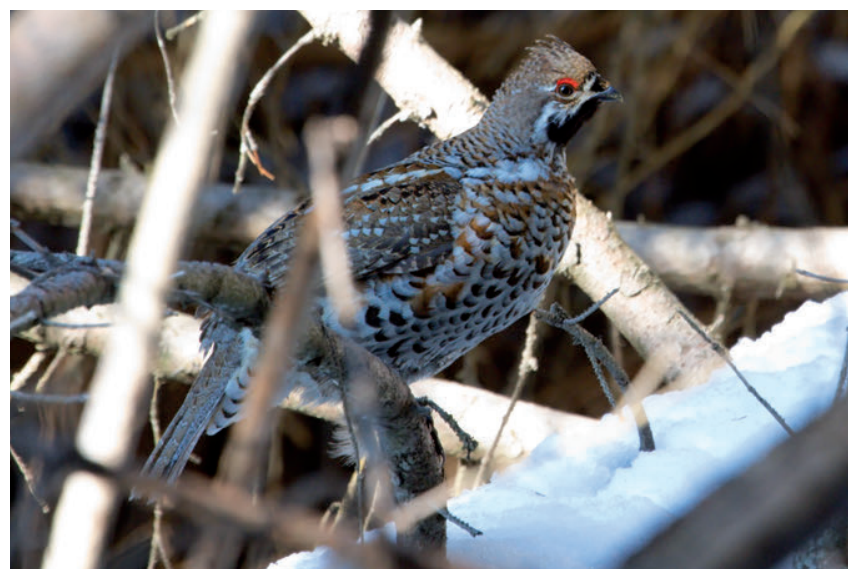

Fig. 7 - Francolino di monte. / Hazel Grouse (Foto / Photo Radames Bionda). 
metodi standard già impiegati in altre aree sull'arco alpino (De Franceschi, 1995). Lo studio ha comportato la realizzazione di censimenti primaverili dei maschi sui punti di canto (arene), con conteggio diretto a distanza dei maschi e delle femmine presenti. Nell'ambito di tale studio è stata stimata la superficie di habitat idonei alla specie presenti nel Parco (praterie naturali, pascoli, boschi di conifere, brughiere, arbusteti, alneti arbustivi e aree con vegetazione arborea sparsa localizzati al di sopra dei 1300 m s.l.m.), sulla base della carta della vegetazione redatta nell'ambito del Piano del Parco; tale superficie potenzialmente idonea è risultata pari a circa 3.000 ha, il $20 \%$ della superficie dell'area protetta. Il censimento primaverile ha permesso di contattare complessivamente 62 individui, di cui: 40 maschi adulti, 1 maschio giovane, 13 maschi di età indeterminata, 7 femmine, 1 individuo indeterminato. Sono state individuate 4 arene con una media di 3,9 maschi per gruppo di parata, oltre a diversi altri siti frequentati da coppie di maschi in atteggiamenti di sfida e stimolo reciproco all'esibizione. La densità complessiva negli ambienti idonei del Parco nel 2009 era di 4,6 maschi/100 ha.

Dal 2010, il personale del CFS esegue monitoraggi primaverili delle aree campione individuate nello studio sopraccitato. Il numero di individui censiti complessivamente è costante anche se sembra esserci una contrazione nel numero di maschi presenti mediamente nelle arene e un aumento delle osservazioni di individui che cantano isolati. Si segnala peraltro la presenza nel 2013 in uno stesso sito di 16 maschi, distribuiti su tre arene di canto.

È verosimile stimare che siano presenti nell'area di studio 40-100 maschi adulti.

\section{Coturnice (Alectoris graeca) SB}

Nell'ambito degli studi preliminari alla stesura del Piano del Parco (EPNVG, 1998) la presenza della specie nell'area protetta veniva valutata come "fortemente limitata", con un numero ridotto di coppie che tendevano a raggrupparsi in autunno in pochissime brigate di piccole dimensioni. Veniva inoltre ipotizzato che le aree di svernamento fossero localizzate verso i Corni di Nibbio (versante meridionale) e quelle di riproduzione nella parte settentrionale del Parco. Nel 1993, 3 territori erano stati rilevati su 115 punti d'ascolto da Movalli \& Grimaldi (1996).

Benché la specie non sia stata oggetto di specifici censimenti all'interno dell'area protetta, negli ultimi anni (2000-2014) la raccolta di dati da osservazioni occasionali da parte di personale del CFS, coordinata dall'Ente Parco, ha permesso di documentare una presenza decisamente più significativa rispetto a quanto veniva indicato in

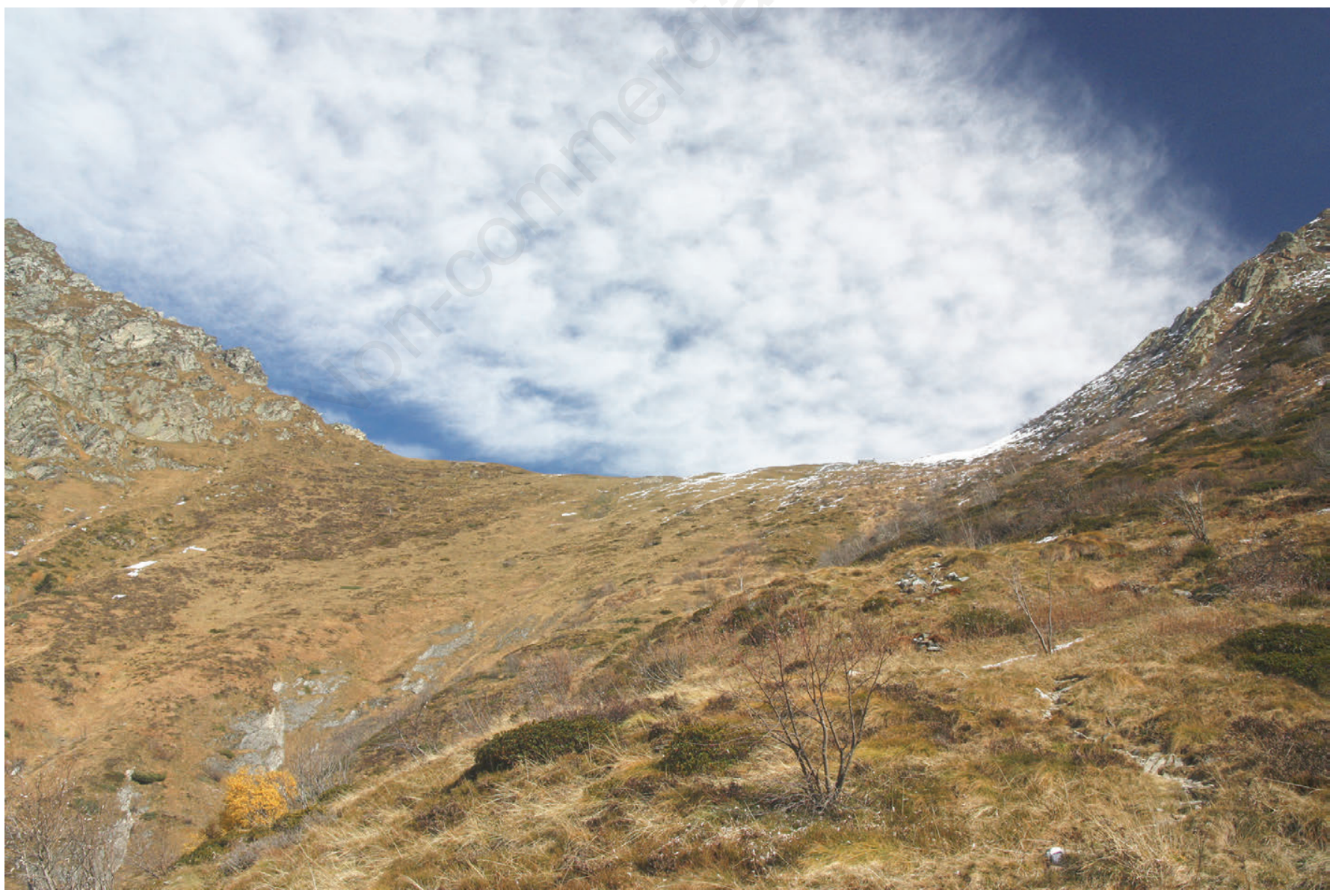

Fig. 8 - Ambienti regolarmente frequentati dal Fagiano di monte presso la Colma di Premosello. / Habitats that are regularly frequented by Black Grouse close to Colma di Premosello. (Foto / Photo Fabio Casale). 
precedenza (EPNVG, 1998). Da tali dati, la coturnice risulta ben distribuita nel Parco Nazionale lungo i crinali posti al di sopra del limite della vegetazione arborea o, a quote più basse, in corrispondenza di aree aperte, anche di ridotta superficie, con praterie rocciose. Nidificazioni accertate: nido con uova a valle delle Strette del Casè a inizio anni 2000: MD; femmina con 4 giovani presso la Cappella di Terza, a 1.800 m s.l.m. il 30/08/2013: AM e Raffaele Marini, com. pers.

In periodo tardo autunnale-invernale la specie è stata rilevata anche a quote basse, in particolare lungo il versante meridionale del Parco (ad esempio, 4 individui il 7/11/2005 all'Alpe I Curt, $900 \mathrm{~m}$, e una nella limitrofa Corpic, $900 \mathrm{~m}$, nella medesima data; 3 individui il 20/01/2014 a Sasso Termine, Capraga; BDVG) ma occasionalmente anche lungo quello settentrionale (ad esempio, 1 individuo il 22/1/2006 a Provola, $900 \mathrm{~m}$; BDVG). L'osservazione di 8 individui nella medesima giornata $(3 / 03 / 2003)$ in periodo non riproduttivo, distribuiti in 4 località differenti lungo il versante settentrionale del Parco (Bocchetta di Vald, Costa Nera, Bocchetta di Scaredi, Bocchetta di Campo) lascia presumere che anche le creste ben esposte di altri settori del Parco, quali il versante meridionale del gruppo Monte Zeda-Pizzo della Marona e del limitrofo Pian Vadà (Fig. 9) siano da includere come aree di svernamento.
D'altro canto, l'aumentato numero di segnalazioni successivamente alla stesura del Piano del Parco è verosimilmente da ascrivere a un maggiore grado di copertura del territorio rispetto agli anni ' 90 , mentre la tendenza che mostra la specie negli ultimi 15 anni, pur in assenza di specifici censimenti, sembra essere negativa.

È verosimile stimare che siano presenti 20-50 coppie nidificanti nell'area di studio.

\section{Quaglia comune (Coturnix coturnix) M irr}

Questo piccolo fasianide frequenta l'area con pochi individui durante le migrazioni, a causa della scarsità di ambienti adatti. Un individuo è stato osservato e fotografato (data non riportata) presso il Passo dei Tre Uomini, in Val Rossa, ed è segnalato sul sito www. in-valgrande.it. In periodo riproduttivo, un individuo in canto territoriale è stato rilevato il 12 ed il 13/6/2001 presso il Bivacco Al Cedo in Valle del Basso (LP).

\section{Fagiano comune (Phasianus colchicus) S}

Un individuo maschio è stato osservato nel 2013 (CM) alle pendici del Monte Carza, verosimilmente proveniente da rilascio per fini venatori. La specie è presente lungo il fondovalle ossolano, limitrofo all'area di studio (Bionda \& Bordignon, 2006).

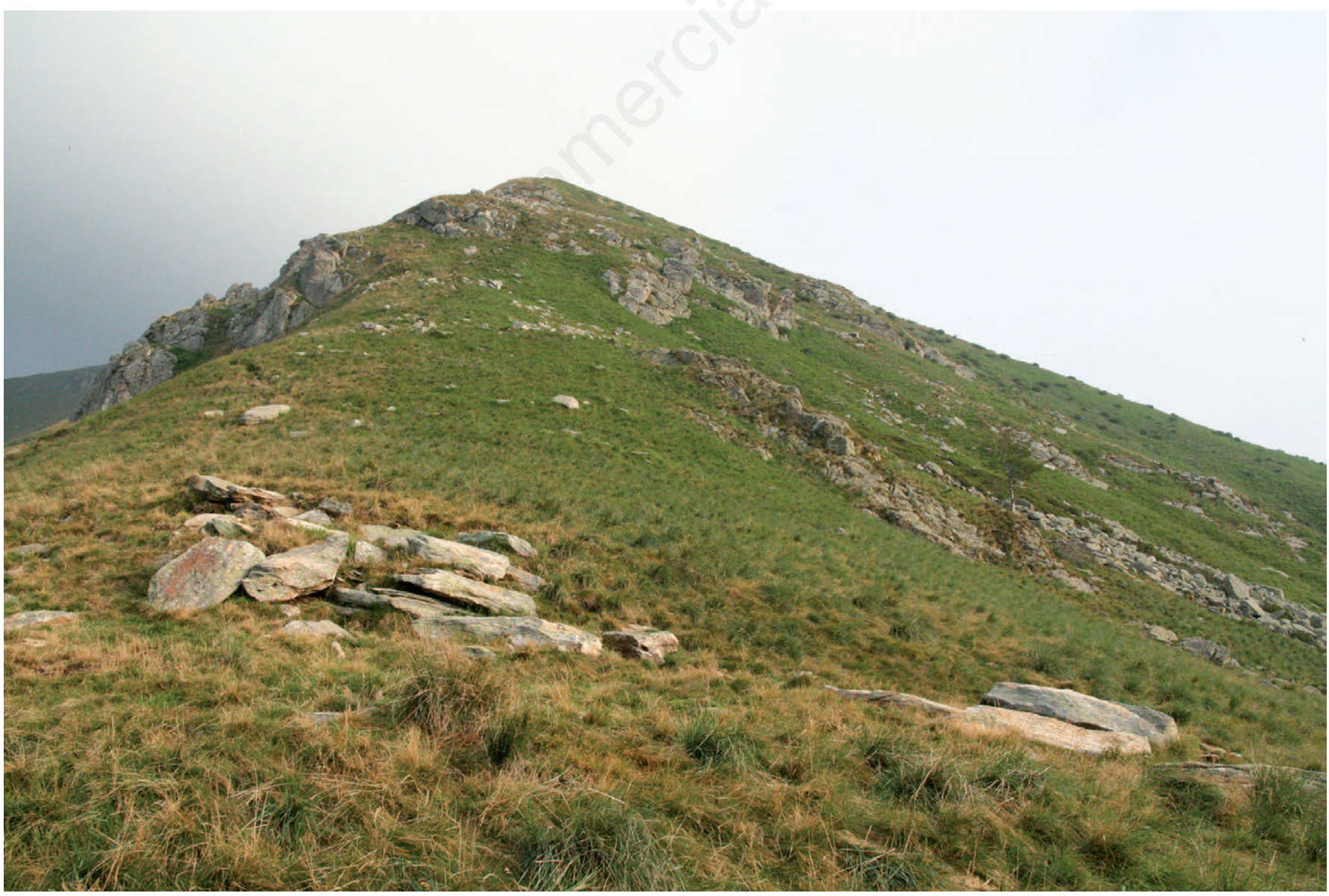

Fig. 9 - Pascoli rocciosi a Pian Vadà, sito di presenza regolare della coturnice. / Rocky pastures at Pian Vadà, a site were Rock Partridge is regularly recorded. (Foto / Photo Fabio Casale). 


\section{PELECANIFORMES}

\section{Phalacrocoracidae}

Cormorano (Phalacrocorax carbo) $\mathrm{M}$ reg

Il cormorano è stato rilevato nell'area di studio solo in transito durante le migrazioni. La prima osservazione nota consiste in 3 individui in volo alto presso la Cima Sasso il 7/05/1994, diretti verso N (FC e Ian Mifsud). In tempi recenti, il regolare transito della specie nel settore orientale dell'area di studio è stato rilevato in periodo primaverile, con gruppi in migrazione lungo la flyway del Verbano. Si segnala, a titolo di esempio, il passaggio osservato dal Monte Carza il 25/03/2014 di 54 individui divisi in 5 gruppi (RB). La specie nidifica regolarmente nella Riserva Naturale Palude Brabbia, nella vicina provincia di Varese (Laddaga \& Casale, 2014), e ha un regolare sito di roosting nella Riserva Naturale di Fondotoce.

\section{CICONIIFORMES}

\section{Ardeidae}

\section{Airone cenerino (Ardea cinerea) M}

Negli ultimi 15 anni la specie viene osservata sempre più regolarmente lungo i principali corsi d'acqua dell'area di studio. Si tratta verosimilmente di individui erratici alla ricerca di cibo, in quanto la specie nidifica da alcuni anni in una garzaia localizzata nei pressi della limitrofa Riserva Naturale di Fondotoce (RB) e ha nidificato nel 2000 anche lungo il fiume Toce, ad Anzola d'Ossola (Bionda \& Bordignon, 2006). Si riportano di seguito alcune osservazioni note:

- nella prima metà degli anni 2000 osservati aironi cenerini in più occasioni risalire o discendere in volo la valle del torrente $\mathrm{S}$. Bernardino all'altezza di Cossogno e Rovegro (ad esempio: 4 individui il 14/06/2002, 1 individuo il 29/06/2004, 1 individuo in alimentazione lungo il Rio Aurelio il 15/02/2005: FC);

- 1 individuo il 24/10/2009 in Val Gabbio (BDVG);

- 1 individuo presso l'Alpe La Piana (Premosello C.) il 18/01/2012 (BDVG);

- 1 individuo in volo sopra la valle del Rio Cannero il 27/03/2014 (FC);

- 1 individuo in volo sopra la confluenza tra Rio Serena e Rio Val Gabbio il 15/04/2014 (BDVG);

- 1 individuo in volo sul Rio Val Rossa il 10/07/2014 (LL);

- 1 individuo osservato più volte durante l'estate 2014 nella zona di confluenza tra Rio Pogallo e Rio Pianezzoli (AM);

- 1 individuo a Pogallo il 16/09/2014 (CM, AM, MP, DR, MD).

\section{FALCONIFORMES}

\section{Accipitridae}

Falco pecchiaiolo (Pernis apivorus) M reg, B Gli studi preliminari alla stesura del Piano del Parco riferivano della presenza della specie in aree limitrofe all'area protetta, in particolare in Valle Vigezzo, mentre non era stata rilevata la presenza nelle vallate interne del Parco, ove la specie veniva valutata come "assente o molto scarsa" (EPNVG, 1998).

Negli anni successivi, in periodo riproduttivo la specie è stata rilevata nei seguenti siti:

- 1 individuo in volo territoriale (a festoni) il 25/05/2004 tra Rovegro e Cossogno (FC);

- 4 individui all'Alpe Prà il 5/07/2007, 2 il 12/07/2007 e 2 il 19/07/2007 (BDVG);

- 2 coppie 1'1/07/2009 nei pressi di Finero (Casale \& Brambilla, 2010b);

- 1 individuo il 16/07/2009 sopra Colloro (Casale \& Brambilla, 2010b) e uno presso Lut (Premosello C.) il 6/07/2013 (FC);

- 1 individuo sul Pizzo Pernice il 19/07/2009 (BDVG);

- 3 individui insieme (una coppia e un maschio) alle pendici del Monte Bavarione, nei pressi di Biogna, il 22/07/2009 (Casale \& Brambilla, 2010b);

- 1 individuo giovane che emetteva continue grida (begging call) nella valle del Rio Cannero il 22/08/2013 (FC, AG);

- 1 individuo adulto che portava un ramo e compiva voli "a festoni" nella valle del Rio Cannero il 24/05/2014 (FC, MP);

- 1 individuo adulto presso il Rifugio Parpinasca (Trontano) l'11/07/2014 (FC).

Tali osservazioni testimoniano l'attuale presenza regolare della specie nell'area di studio, con una distribuzione riferibile soprattutto agli ambienti più aperti.

La presenza nell'area di studio è stimabile in 7-10 coppie nidificanti.

L'area è anche interessata da un flusso migratorio primaverile ed uno autunnale della specie, quest'ultimo apparentemente meno significativo in termini numerici. In primavera, si segnalano le seguenti osservazioni dal Monte Carza:

- 4 individui l' $8 / 05 / 2013$ verso $\mathrm{N}$ (FC);

- 1 individuo il 5/05/2014 verso N (FC, MP);

- 12 individui il 17/05/2014 verso N (FC, MP);

- 14 individui il 24/05/2014 verso N (FC, MP);

- 5 individui il 28/05/2014 (MP).

Inoltre, per altre località, sono segnalati:

- 6 individui in migrazione all'Alpe Prà il 24/05/2012 (BDVG);

- 11 individui verso N 1'8/05/2013 presso il Rifugio Pian Cavallone (FC);

- 4 individui verso $\mathrm{S}$ in località Cresta della Ciresa il 22/08/2013 (FC, AG);

- 1 individuo verso $\mathrm{S}$ dalla Cima di Morissolo il 22/08/2013 (FC, AG);

- 2 individui verso S dalla vetta del Monte Spalavera il 22/08/2013 (FC, AG ); ,

- 1 individuo verso $\mathrm{S}$ dal Monte Limidario il 14/08/2014 (FC, MB);

- 1 individuo molto scuro verso $\mathrm{S}$ a Scaredi 1'8/09/2014 (FC, MP). 
Nibbio bruno (Milvus migrans) M reg, B

Gli studi preliminari alla stesura del Piano del Parco (EPNVG, 1998) avevano portato all'individuazione di una probabile coppia a Beura-Cardezza ed una tra il Parco e il Lago Maggiore (CM). Attualmente, stanti le numerose osservazioni della specie in periodo riproduttivo tra il 2008 e il 2014, una coppia nidificante potrebbe essere presente lungo il versante meridionale dell'area protetta alle quote più basse, lungo i versanti tra le frazioni di Mergozzo (Albo, Candoglia e Nibbio) e l'abitato di Cuzzago (CM). La specie troverebbe il suo habitat ideale nei settori sudorientale ed orientale dell'area di studio, in particolare lungo i tratti terminali del torrente San Bernardino (FC) e del fiume Toce e lungo le rive del Lago Maggiore (da Feriolo a Cannobio), dove è presente con 3-5 coppie.

L'area è anche interessata da un flusso migratorio primaverile ed uno autunnale, quest'ultimo apparentemente meno significativo in termini numerici. Dal Monte Carza sono stati rilevati i seguenti individui in transito:

- 4 individui verso $\mathrm{N}$ il 17/04/2014 (FC);

- 1 individuo verso $\mathrm{N}$ il 5/05/2014 (FC, MP);

- 2 individui verso N il 17/05/2014 (FC, MP);

- 2 individui verso $\mathrm{N}$ il 24/05/2014 (FC, MP);

- 1 individuo verso $\mathrm{N}$ il 28/05/2014 (MP).

Altre osservazioni in periodo migratorio:

- 3 individui dalla Cresta della Ciresa il 6/04/2013 (RB, FC, CM, DF, FCL, PP, LP);

- 3 individui diretti verso $\mathrm{N}$ presso il Rifugio Pian Cavallone l'8/05/2013 (FC);

- 1 individuo dalla Cresta della Ciresa il 22/8/2013 (FC, AG).

Nibbio reale (Milvus milvus) M reg

Il nibbio reale viene osservato occasionalmente nell' area di studio, soprattutto durante la migrazione primaverile. Di seguito si riportano le osservazioni sinora note:

- 2 individui il 24/03/2002 a Cossogno diretti verso $\mathrm{N}(\mathrm{FC})$;

- 1 individuo al Passo di Folungo diretto verso $\mathrm{N}$ il 7/05/2009 (BDVG);

- 5 individui dal Monte Carza diretti verso N il 12/04/2013 (RB, FC);

- 1 individuo dal Monte Carza diretto verso $\mathrm{N}$ il 27/03/2014 (FC).

\section{Gipeto (Gypaetus barbatus) M}

Nella Banca Dati della Rete Osservatori Alpi Occidentali, che coordina attività di raccolta dati, monitoraggio e sensibilizzazione sul progetto di reintroduzione del gipeto sull'arco alpino occidentale, risultano 4 osservazioni di questa specie nell'area di studio, tutte relative agli anni ' 90 del XX secolo:

- 1 adulto il 18/07/1995 sopra Colloro (R. Tucci);

- 1 immaturo (Republic 5 o 6) il 21/04/1996 sopra Marone, in Val Vigezzo (I. Franzoni) e il medesimo individuo l'1/05/1996 presso l'Alpe Corone (Vogogna) (E. Sesone);

- 1 individuo il 7/09/1999 presso il Pizzo Stagno (C. Damiano).

Più recentemente è stato osservato un individuo adulto il 10/09/2014 sopra Piedimulera (FCA).
Grifone (Gyps fulvus) M

Nel Parco la specie, molto mobile e caratterizzata da frequenti erratismi, compare occasionalmente, soprattutto negli ultimi anni:

- 1 individuo è stato osservato il 31/05/2009 sul Pizzo Faiè (Giuseppe Niccolini, com. pers.);

- 1 immaturo, proveniente da Pian Vadà e diretto verso $\mathrm{W}$, è stato osservato sul Monte Zeda il 21/06/2012 (FC, MB);

- 1 individuo (Fig. 10) è stato osservato in più occasioni tra Cima della Laurasca, Scaredi e Pizzo Stagno in date diverse tra la fine di luglio e i primi di agosto 2013, attirato dalla presenza dei corpi di 66 pecore uccise da un fulmine (vari osservatori, tra i quali CM, LL, LP e Raffaele Marini, com. pers.).

\section{Biancone (Circaetus gallicus) M reg, B}

Nell'area di studio la specie (Fig. 11) è nidificante almeno dall'inizio degli anni 2000 con 4-5 coppie, così distribuite:

- 1 coppia nel settore meridionale (versanti sopra Premosello-Chiovenda);

- 1-2 coppie nel settore occidentale, lungo il versante sinistro della Val d'Ossola compreso tra il Parco e il fiume Toce;

- 1 coppia nel settore settentrionale, a cavallo tra Parco e Valle Cannobina;

- 1 coppia nel settore orientale, tra Parco e Lago Maggiore.

La nidificazione è stata accertata nel 2013, con l'osservazione di un adulto e due giovani da poco involati il 12/07/2012 nei pressi di Pian Vadà (FC).

L'area è anche interessata da un flusso migratorio primaverile; si segnala, ad esempio, 1 individuo in migrazione verso N sul Monte Carza il 24/03/2014 (RB) e 1 individuo il 5/05/2014 (FC, MP).

Falco di palude (Circus aeruginosus) M reg

La specie viene regolarmente osservata nell'area di studio durante le migrazioni primaverile e autunnale. Di seguito le segnalazioni note:

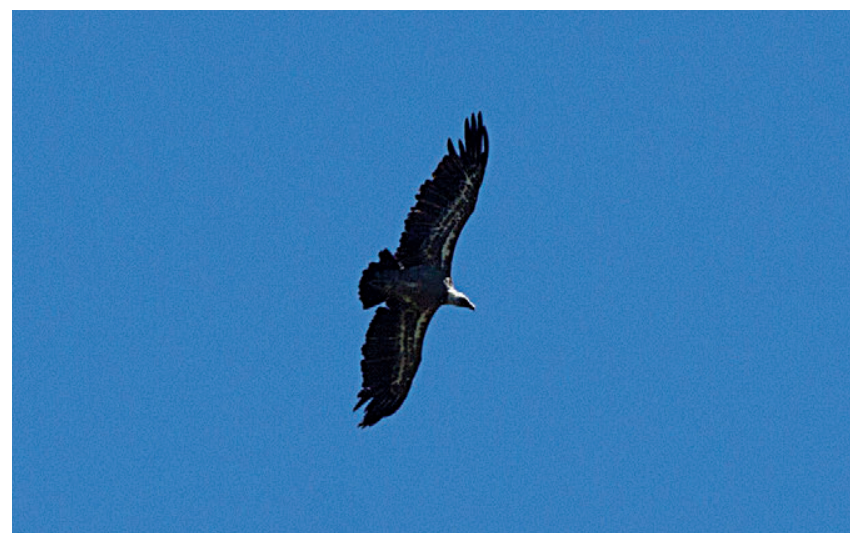

Fig. 10 - Grifone osservato a luglio-agosto 2013 a Scaredi. / Griffon Vulture that was observed in July-August 2013 at Scaredi. (Foto / Photo Raffaele Marini). 
- 1 individuo in migrazione sopra Vogogna nella prima metà degli anni 2000 (RB);

- 3 individui osservati dalla Cresta della Ciresa diretti a N il 6/04/2013 (RB, FC, CM, FCL, DF, PP, LP);

- 5 individui diretti a N il 12/04/2013 sul Monte Carza (RB, FC);

- 1 femmina diretta a N 1'8/05/2013 nei pressi del Rifugio Pian Cavallone (FC);

- 1 maschio diretto a N il 5/05/2014 sul Monte Carza (FC, MP);

- 1 individuo diretto a N il 17/05/2014 sul Monte Carza (FC, MP);

- 1 immaturo diretto a N il 24/05/2014 sul Monte Carza (FC, MP);

- 5 individui diretti a S a Scaredi l'8/09/14 (FC, MP);

- 1 individuo diretto a S a Scaredi il 14/09/14 (Raffaele Marini, com. pers.; Fig. 12);

- 1 individuo diretto a S a Scaredi il 20/09/14 (BDVG).

Albanella reale (Circus cyaneus) $\mathrm{M}$ reg, $\mathrm{W}$ reg

I versanti esposti a sud caratterizzati da ambienti aperti e, almeno parzialmente, non innevati sono idonei a ospitare la specie sia durante le migrazioni, sia come svernante. Per l'area di studio sono note le seguenti osservazioni in periodo tardo autunnale-invernale:

- 1 femmina il 30/11/1986 a Cappella di Terza (Malesco), con terreno parzialmente innevato (FC, E. Giussani);

- 1 femmina all'Alpe Pala (Miazzina) il 7/02/2002 (FC);

- 1 maschio sopra Piaggia il 9/02/2007 (AM);

- 1 femmina a Pian Vadà il 12/11/2010 (CM, AP, Giulio Silvestrini, Giovanni Mastrobuoni);

- 1 femmina all'Alpe Suì il 21/01/2011, fatta oggetto di mobbing da corvi imperiali (BDVG).

- 1 maschio in migrazione verso $\mathrm{N}$ è stato osservato dalla Cresta della Ciresa il 6/04/2013 (RB, FC, CM, FCL, DF, PP, LP).

Albanella minore (Cyrcus pygargus) $\mathrm{M}$ irr

La specie è stata osservata solo in due occasioni nell'area di studio, in entrambi i casi durante la migrazione primaverile:

- 1 femmina in località Alpe Ompio, diretta verso W, il 24/04/2013 (FC, LL),

- 1 femmina sul Monte Carza, diretta verso N, il 17/04/2014 (FC, CM).

\section{Astore (Accipiter gentilis) SB, M reg}

L'area di studio ospita vaste aree boscate e poco disturbate, idonee alla specie. Di seguito vengono forniti alcuni dati relativi al periodo riproduttivo:

- 1 coppia con maschio molto allarmato il 10/04/2002 all'Alpe Pala (Miazzina; FC);

- 1 adulto a Aurano il 16/04/2000 (FC) e il 21/07/2009 (Casale \& Brambilla, 2010b);

- 1 maschio adulto all'Alpe Ompio il 19/05/2001 (FC);

- 1 individuo in canto sotto Colloro il 3/03/2012 (BDVG);
- 1 individuo presso il Monte Todum il 18/05/2013 (FC);

- 1 individuo sul Monte Bavarione il 25/06/2013 (FC);

- 1 individuo presso l'Alpe Archìa il 03/07/14 (BDVG);

- 1 individuo in canto nella pecceta presso Cappella Porta (Caprezzo) il 20/03/2014 (FC, LL);

- 1 individuo presso il Monte Carza il 20/03/2014 (FC, LL) e in volo territoriale il 17/04/14 (FC, CM);

- 1 individuo il 3/07/2014 presso l'Alpe Boschelli (LL, MP).

Si stima la presenza di 5-10 coppie nidificanti nell'area di studio.

Il settore verbanese è inoltre interessato da un flusso migratorio primaverile, verosimilmente legato alle uniche popolazioni europee migratrici, nidificanti in Scandinavia e Russia (del Hoyo et al., 1994):

- 5 individui diretti a $\mathrm{N}$ sulla Cresta della Ciresa il 6/04/2013 (RB, FC, CM, FCL, DF, PP, LP);

- 10 individui diretti a $\mathrm{N}$ sul Monte Carza il 12/04/2013 (RB, FC);

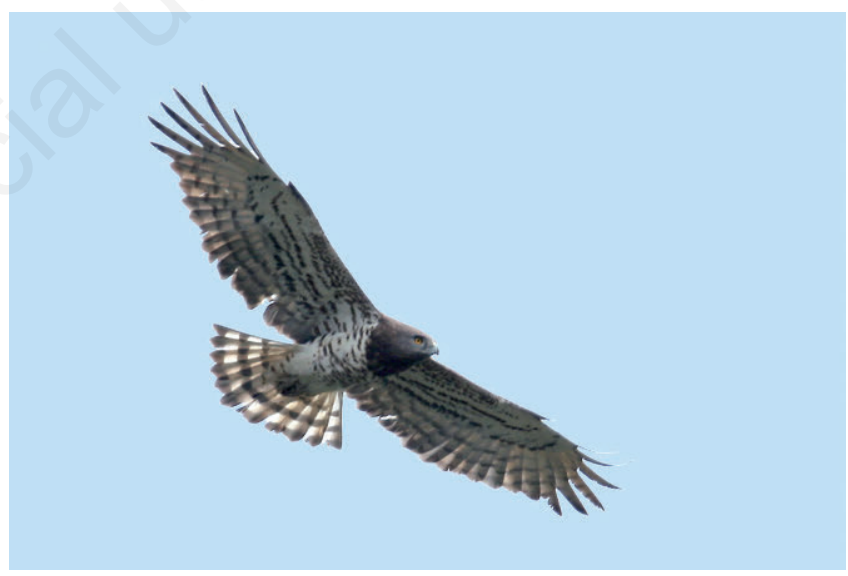

Fig. 11 - Biancone. / Short-toed Eagle. (Foto / Photo Antonello Turri).

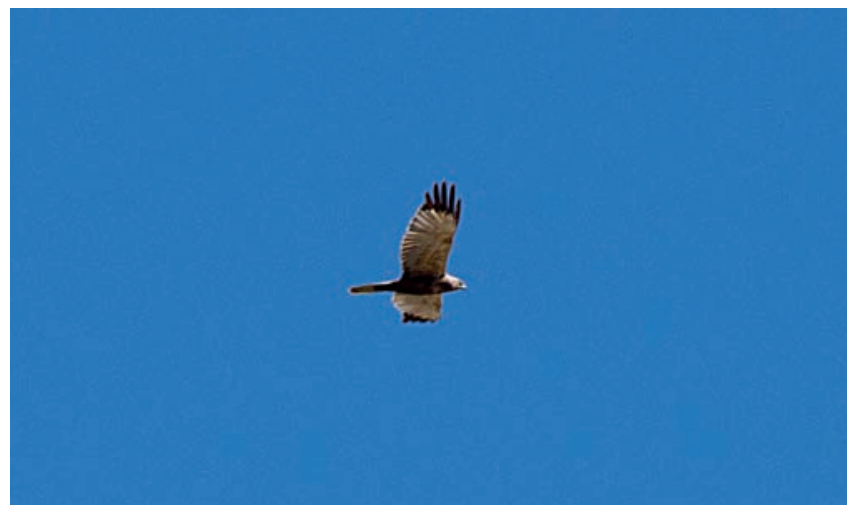

Fig. 12 - Falco di palude in transito a Scaredi il 14/09/2014. / Marsh Harrier passing above Scaredi on 14/09/2014. (Foto / Photo Raffaele Marini). 
- 2 individui diretti a N sul Monte Carza il 17/05/2014 (FC, MP).

In autunno, si segnala un individuo in migrazione verso S sul Monte Carza il 14/09/2014 (MP).

Un immaturo è stato osservato il 7/11/2014 sopra l'Alpe La Motta mentre tentava, senza successo, di catturare una tordela in ambiente di faggeta (FC).

\section{Sparviere (Accipiter nisus) SB, M reg}

Malgrado l'ampia disponibilità di aree boscate potenzialmente idonee, nell'ambito delle indagini ornitologiche condotte negli ultimi anni (2009-2014) la specie è apparsa essere meno comune come nidificante rispetto a quanto indicato nel Piano del Parco: "Il Parco della Val Grande ospita una popolazione di sparviere relativamente ben distribuita all'interno dei confini" (EPNVG, 1998: pag. 165). Un solo territorio era stato inoltre rilevato nel 1993 su 115 punti d'ascolto da Movalli \& Grimaldi (1996). Una possibile spiegazione consiste nel fatto che l'area di studio, a causa della copertura nevosa, risulta in gran parte inaccessibile nel periodo di massima contattabilità della specie (inizio della primavera). Negli anni successivi, la presenza dello sparviere in periodo riproduttivo è stata rilevata nei seguenti siti:

- 1 individuo tra Aurano e Alpe Colle ed 1 presso Scareno il 16/04/2000 (FC);

- 1 individuo il 25/06/2001 sopra Cossogno intento a fare oggetto di mobbing una Poiana (FC);

- 1 individuo presso Alpe La Piana (Premosello C.) il 20/03/2004 (FC);

- 1 individuo all'Alpe I Curt (Premosello C.) il 2/07/2009 fatto oggetto di mobbing da Rondine montana (Ptyonoprogne rupestris) (Casale \& Brambilla, 2010b);

- 1 individuo al Pian dei Sali (Malesco) il 10/04/2011 (FC);

- 1 individuo in alta Val Loana il 22/07/2011 (FC, CM, LL);

- 1 individuo a $1.900 \mathrm{~m}$ tra Monte Zeda e Pizzo della Marona il 29/06/2012 (FC);

- 1 individuo a monte di Cappella Porta il 22/04/2013 (FC, CM);

- 1 individuo che fa oggetto di mobbing un astore a W del Monte Carza il 12/04/2013 (FC, RB);

- 1 individuo a $1.800 \mathrm{~m}$ presso la Colma di Premosello il 3/07/2013 (FC, CM);

- 1 individuo tra In La Piana e Alpe Boschelli il 9/06/2014 (LL, MP);

- 1 individuo a $1.950 \mathrm{~m}$ a caccia di individui di Spioncello (Anthus spinoletta) sul Cimone di Straolgio il 30/06/2014 (FC);

- 1 individuo a In La Piana il 3/07/2014 (LL, MP);

- 1 individuo a Pian di Boit il 17/07/2014 (LL, MP).

Si stima la presenza di 15-20 territori nell'area di studio.

Durante la migrazione primaverile è stato osservato in sorvolo sul Monte Carza nelle seguenti date:

- 7 individui diretti a N il 12/04/2013 (RB, FC);

- 2 individui diretti a $\mathrm{N}$ il 27/03/2014 (FC);

- 1 individuo diretto a $\mathrm{N}$ il 17/04/2014 (FC, CM);

- 1 individuo diretto a N il 5/05/2014 (FC, MP).
Durante la migrazione autunnale si segnala il transito di 3 individui il 18/10/2014 a Scaredi, diretti verso S (FC, MP).

\section{Poiana (Buteo buteo) SB, M reg}

Specie piuttosto comune come nidificante nell'area di studio. Cinque territori erano stati rilevati nel 1993 su 115 punti d'ascolto da Movalli \& Grimaldi (1996), per una dominanza relativa dello 0,3\%, e negli anni successivi (in particolare nel 2013-2014) la sua presenza in periodo riproduttivo è stata rilevata tra Cicogna e Pogallo (una coppia osservata in più occasioni; FC, CM, MP, LL, AM, ST) e nei seguenti altri siti:

- 1 adulto che trasporta una preda (colubride di circa un metro di lunghezza) l'01/07/2001 e una coppia che difende il territorio il 24/03/2002 a Cossogno (FC);

- una coppia a Cappella Porta (Caprezzo) 1'8/05/2013 (FC);

- 2 adulti in scontro territoriale a Ponte Casletto il 31/05/2013 (FC);

- 1 individuo che fa oggetto di mobbing un falco pecchiaiolo a Cicogna il 31/05/2013 (FC);

- 3 individui a Colloro il 18/07/2013 (BDVG);

- una coppia il 17/05/2014 (uno dei due cattura un ramarro e si allontana con la preda) e due coppie con voli territoriali a "festoni" il 24/05/2014 sul Monte Carza (FC, MP);

- 1 individuo sulla Cima Mottone (CavaglioSpoccia), 1 in loc. Motta (Falmenta) e 1 sopra Gurro il 5/06/2014 (FC) e un giovane che emette continue grida (begging calls) sopra Spoccia il 14/08/2014 in Valle Cannobina (FC, MB);

- 1 individuo a In La Piana il 3/07/2014 (LL, MP).

Si stima la presenza di $10-15$ territori nell'area di studio.

In migrazione:

- 9 individui verso $\mathrm{N}$ il 6/04/2013 sulla Cresta della Ciresa (RB, FC, CM, FCL, DF, PP, LP);

- 11 individui verso N il 12/04/2013 sul Monte Carza (RB, FC);

- 2 individui verso N 1'8/05/2013 dal Rifugio Pian Cavallone (FC);

- 4 individui verso N il 5/05/2014 sul Monte Carza (FC, MP).

Aquila minore (Aquila pennata) A-1 (2014)

Un sola segnalazione nota per l'area di studio: 2 individui osservati e fotografati (Fig. 13) il 28/05/2014 in migrazione nel settore orientale dell'area di studio (MP).

Aquila reale (Aquila chrysaetos) SB

Gli studi preliminari alla stesura del Piano del Parco avevano portato all'individuazione di 3 territori ricadenti nell'area protetta (EPNVG, 1998). Dalla successiva indagine specifica svolta da Bionda (2003a) su tutto il territorio del Verbano Cusio Ossola (VCO) e nei successivi aggiornamenti (Bionda \& Bordignon, 2006; Banca Dati della Società di Scienze Naturali del VCO; BDVG), alla metà degli anni 2000 era nota la presenza di 6 territori di aquila reale nel Parco e nelle aree limitrofe 
(Fig. 14). Questa situazione potrebbe essere ulteriormente mutata, come suggerisce il recente insediamento di un nuovo territorio (2014) nel settore orientale dell'area di studio (RB, FC, CM, MP). Durante l'indagine condotta nel 2003, nei 6 territori erano stati individuati da 1 fino a 5 nidi per coppia (Bionda, 2003a).

\section{Pandionidae}

Falco pescatore (Pandion haliaetus) M irr

La presenza della specie nell'area di studio è stata rilevata solo in poche occasioni, durante le migrazioni:

- 1 individuo al Passo Folungo diretto a $\mathrm{N}$ all'inizio degli anni 2000 (ST);

- 1 individuo posato sul tetto di un edificio nel centro storico di Vogogna, intento a cibarsi di una trota, il 3/11/2011 (Marta Petrizzi, com. pers.; Fig. 15);

- 2 individui dalla Cresta della Ciresa diretti verso N il 6/04/2013 (RB, FC, CM, FCL, DF, PP, LP);

- 1 individuo sul Pizzo Faiè diretto a W il 13/04/2013 (CM);

- 1 individuo sul versante valgrandino di PremoselloChiovenda il 19/09/2014, diretto a W (AP).

\section{Falconidae}

Gheppio (Falco tinnunculus) SB, M reg

La specie (Fig. 16) è diffusa soprattutto alle quote più alte dell'area di studio, al di sopra del limite della vegetazione arborea. Nel 1993, nel Parco erano state rilevate 3 coppie su 115 punti d'ascolto, per una dominanza

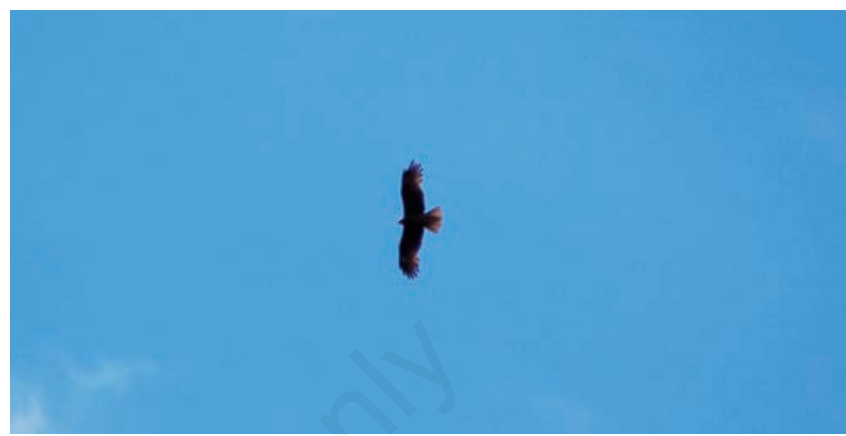

Fig. 13 - Aquila minore osservata il 28/05/2014. / Booted Eagle observed on 28/05/2014. (Foto / Photo Manuel Piana).
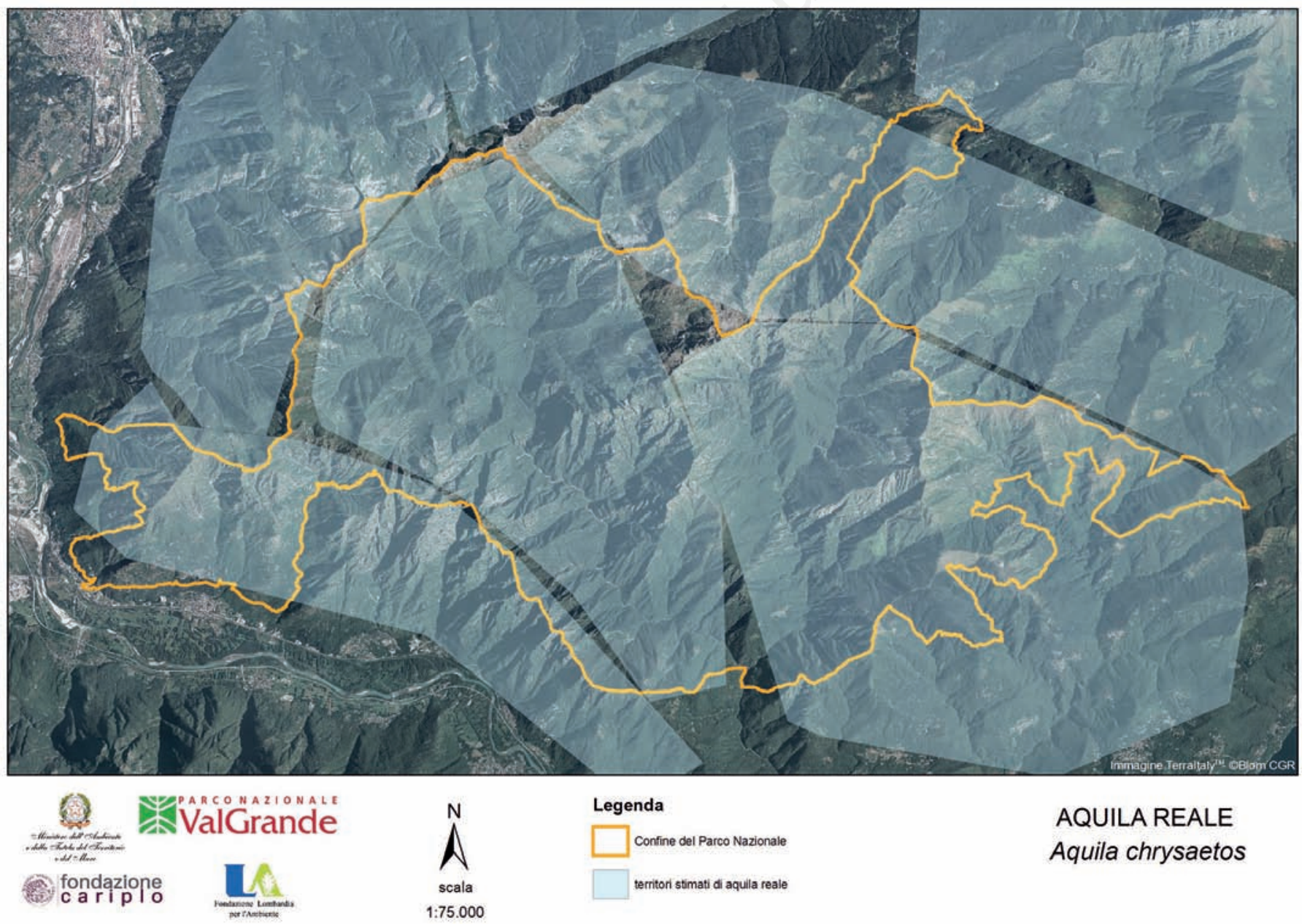

AQUILA REALE Aquila chrysaetos

Fig. 14 - Delimitazione presuntiva dei territori di Aquila reale nel Parco e aree limitrofe, definiti sulla base di osservazioni simultanee. / Estimated Golden Eagle territories in the Park and surrounding areas, defined by simultaneous observations. (Bionda, 2003a) 
relativa dello 0,2\% (Movalli \& Grimaldi, 1996). Negli anni più recenti (2013-2014) si segnalano i seguenti dati di presenza in periodo riproduttivo:

- Alpe Corpic (Premosello C.): 1 individuo il 15/04/2013 (BDVG);

- Colloro: 1 individuo il 22/04/2013 (BDVG);

- dorsale Monte Todano-Il Pizzo: 1 femmina l'8/05/2013 (FC) ed 1 tipo femmina l'8/05/2014 (FC);

- tra Colma di Premosello e Alpe Serena: 1 individuo 1'11/06/2013 lungo i versanti a Ovest (FC, LL, CM, PD);

- Bocchetta di Terza, in alta Val Pogallo: 1 maschio che fa oggetto di mobbing un'aquila reale il 17/06/2013 (FC, CM, ST);

- Pian d'Arla (Aurano): 1 individuo il 25/06/2013 (FC);

- Cima della Laurasca: 1 maschio che fa oggetto di mobbing un'aquila reale giovane 1'11/07/2013 (FC, CM);

- Monte Carza: una coppia il 24/03/2014 e nei mesi successivi (FC, MP);

- Alpe La Piana (Premosello C.): 2 giovani il
4/07/2014 (LL);

- Bivacco Pian Vadà: coppia con 2 giovani dell'anno che emettono grida (begging call) il 18/06/2014 (FC);

- Cimone di Straolgio: 2 individui il 13 e il 30/08/2013 (AM), 1 maschio il 30/06/2014 (FC), 1 maschio che fa oggetto di mobbing un'aquila reale l'11/09/2014 (AM);

- Alpe Menta: coppia che fa oggetto di mobbing un'aquila reale 1'11/07/2014 (FC, MD, DR, FCA);

- Alpe Spoccia, in Val Cannobina: una coppia il 14/08/2014 (FC, MB).

Si stima la presenza di $15-20$ coppie nidificanti nell'area di studio.

In periodo migratorio si segnalano i seguenti dati:

- 1 individuo dalla Cresta della Ciresa diretto a $\mathrm{N}$ il 6/04/2013 (RB, FC, CM, FCL, DF, PP, LP);

- 30 individui dal Monte Carza diretti a N il 12/04/2013 (RB, FC);

- 2 individui dal Monte Carza diretti a N l'8/05/2013 (FC);

- 1 individuo diretto a $\mathrm{N}$ sulla dorsale tra Pizzo Pernice e Monte Todano 1'8/05/2014 (FC).

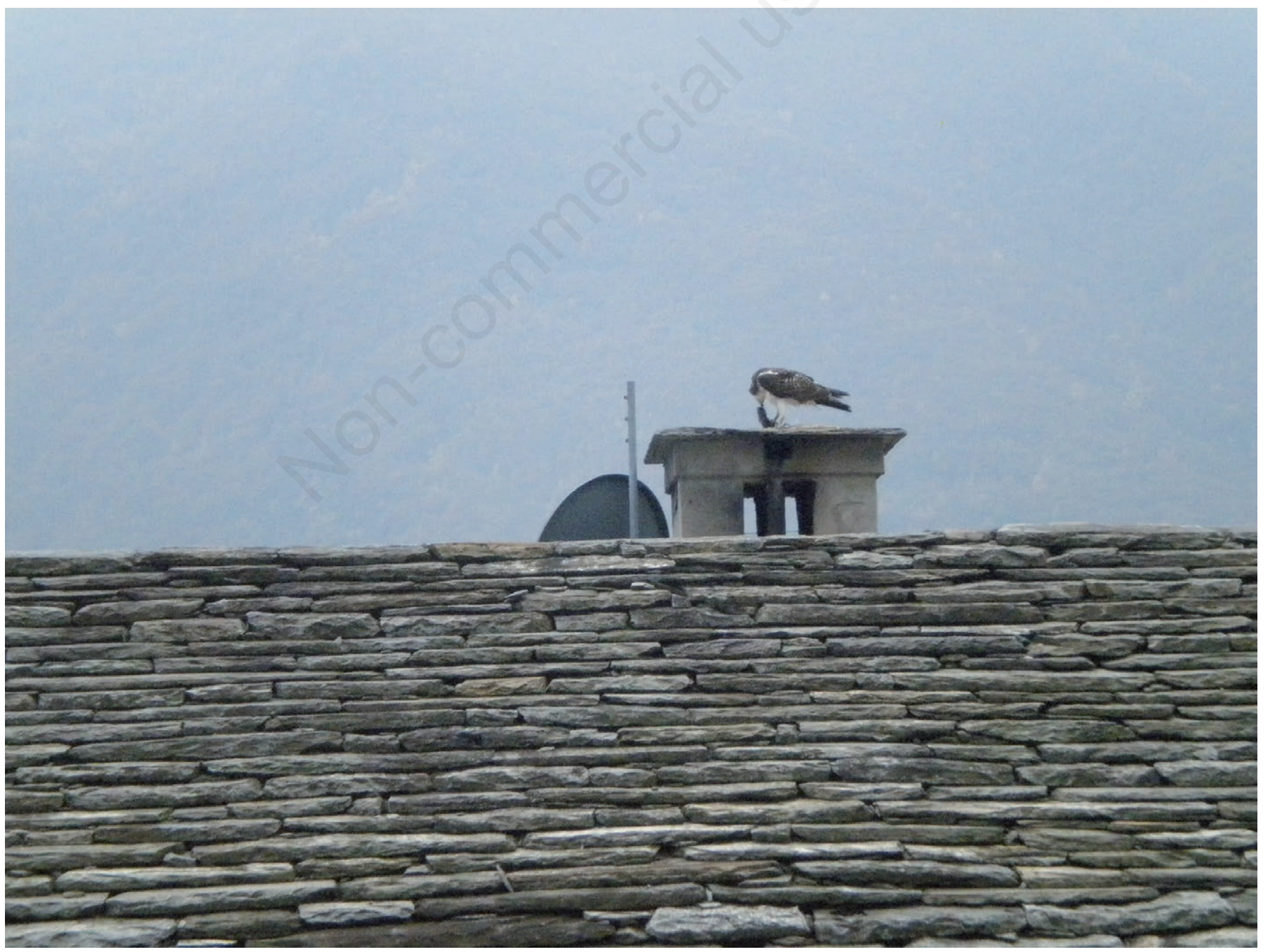

Fig. 15 - Falco pescatore a Vogogna il 3/11/2011. / Osprey in Vogogna on 3/11/2011, eating a trout on a roof. (Foto / Photo Marta Petrizzi). 
- 2 individui dal Monte Carza diretti a N il 17/04/2014 (FC, CM);

- 1 individuo diretto a S alla Colma di Premosello il 16/09/2014 (FC).

Alcuni individui occasionalmente svernano nell'area, con terreno per nulla o scarsamente innevato: ad es. 2 individui il 4/12/1990 in alta Val Loana (FC).

\section{Smeriglio (Falco colombarius) M irr}

Una sola osservazione nota per l'area di studio: un maschio osservato da Scaredi il 25/10/2012 mentre percorreva, in attività di caccia, le pendici meridionali del Cimone di Straolgio e successivamente attraversava la Val Grande in direzione del Monte Pedum (FC).

\section{Lodolaio (Falco subbuteo) M reg}

Nell'area di studio la specie è stata rilevata solo durante le migrazioni. Durante l'attività di monitoraggio della migrazione primaverile dei rapaci diurni condotta nel 2013 e 2014 sono state effettuate le seguenti osservazioni:

- 5 individui (3 giovani e 2 adulti) dal Monte Carza diretti a N il 12/04/2013 (RB, FC);

- 1 individuo in sorvolo verso $\mathrm{N}$ sulla dorsale tra Pizzo Pernice e Monte Todano (cattura prede mentre vola) l' $8 / 05 / 2013$ (FC);

- 1 giovane dal Monte Carza diretto a N il 17/04/2014 (FC, CM);

- 1 giovane dal Monte Carza diretto a N il 5/05/2014 (FC, MP);

- 1 individuo in sorvolo verso $\mathrm{N}$ sulla dorsale tra Pizzo Pernice e Monte Todano 1'8/05/2014 (FC);

- 1 individuo dal Monte Carza diretto a $\mathrm{N}$ il 28/05/2014 (MP);

- 1 adulto dal Monte Carza diretto a N il 5/06/2014 (FC, MP).

A queste si aggiunge l'osservazione antecedente il periodo indicato di un individuo in migrazione autunnale nei pressi di Vogogna il 9/09/2010 (BDVG).

Falco pellegrino (Falco peregrinus) SB, M reg

Nel Parco sono stati rilevati 3 territori su 115 punti

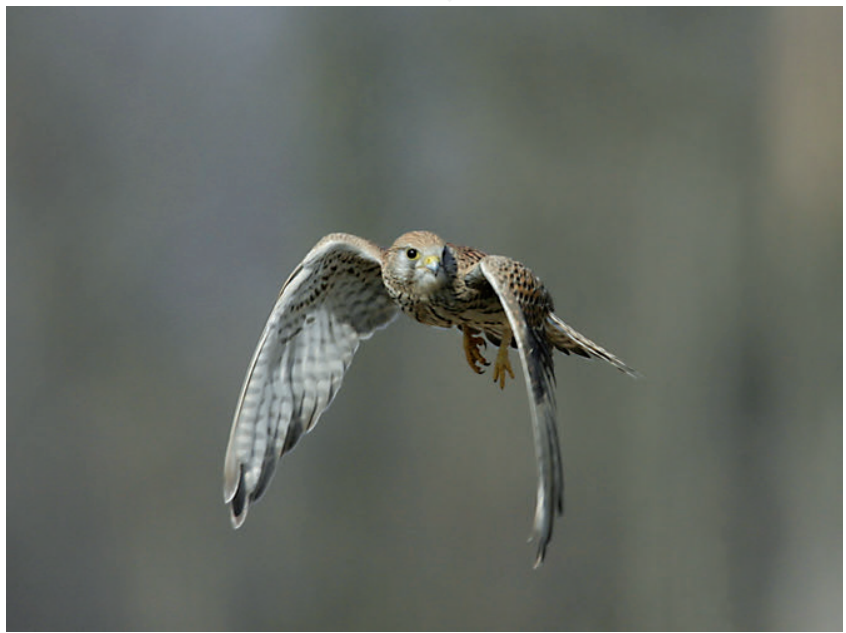

Fig. 16 - Gheppio. / Eurasian Kestrel. (Foto / Photo Antonello Turri). d'ascolto nel 1993, per una dominanza relativa dello 0,2 \% (Movalli \& Grimaldi, 1996). Allo stato attuale delle conoscenze, il Falco pellegrino nidifica con una coppia all'interno dei confini del Parco, nel suo settore meridionale, ma l'area di studio è complessivamente interessata dalla presenza di 4-5 territori, ovvero:

- una seconda coppia nidificante nel settore meridionale (Bionda, 2003b);

- almeno una coppia presente nel settore orientale;

- una-due coppie nel settore settentrionale, tra Valle Vigezzo e Valle Cannobina.

In un'occasione, un falco pellegrino è stato osservato dalla sponda lombarda del Lago Maggiore attraversare il lago e recarsi nella zona di Pian Vadà, verosimilmente in caccia (Fabrizio Sergio, com. pers.).

\section{CHARADRIIFORMES}

\section{Charadriidae}

Piviere tortolino (Charadrius morinellus) A-1 (2014)

Una sola osservazione nota per questa specie. Si tratta di un adulto in abito nuziale, dal comportamento tipicamente confidente, sul Monte Todano, sopra il Rifugio Pian Cavallone, osservato e fotografato 1' 1/05/2014 (Piero Dosso e Walter Meneghin, com. pers.; Fig. 17).

\section{Scolopacidae}

Beccaccino (Gallinago gallinago) M irr, W irr

Specie che frequenta l'area di studio solo occasionalmente durante le migrazioni (in particolare quella autunnale, in quanto in primavera gli ambienti idonei alla sosta sono verosimilmente sempre coperti dalla neve) e lo svernamento (quest'ultimo avviene alle basse quote). Sono noti i seguenti dati di presenza:

- 1 individuo all'Alpe Sciarina (Cuzzago) il 29/12/2010 (BDVG);

- 1 individuo al Ponte Luetto (Premosello C.) il 29/12/2011 (BDVG);

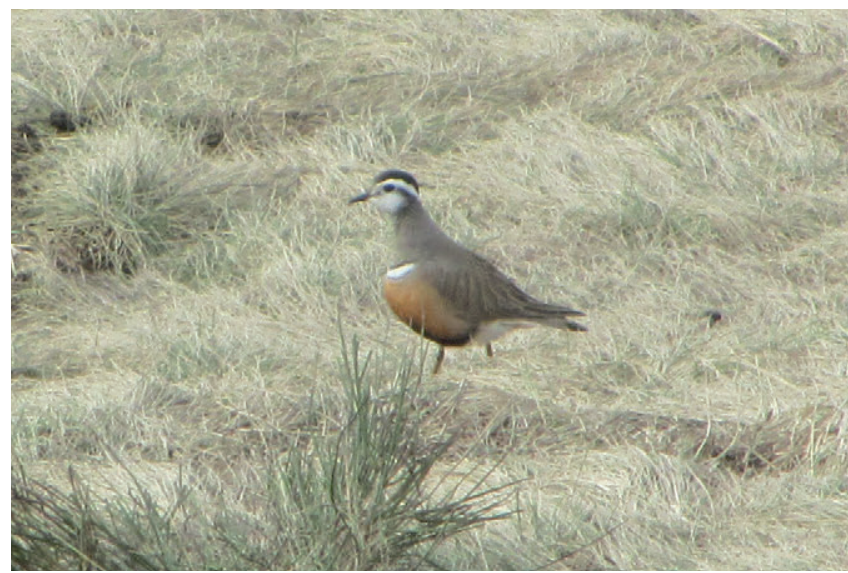

Fig. 17 - Piviere tortolino sul Monte Todano 1'1/05/2014. / Dotterel on Monte Todano on 01/05/2014. (Foto / Photo Piero Dosso e Walter Meneghin). 
- 1 individuo all'Alpe Pieso il 5/11/2013 (BDVG);

- 5 individui all'Alpe Lagarasc il 5/11/2013 (BDVG);

- 1 individuo all'Alpe Morgagno 1'8/11/2013 (BDVG).

Beccaccia (Scolopax rusticola) M reg, B

Specie elusiva, per la quale sono noti pochi dati di presenza nell'area di studio:

- nido con 4 uova rinvenuto il 17/05/1989 a $1.300 \mathrm{~m}$ in Val Cannobina (G.P.S.O., 1990);

- 1 individuo il 17/06/2000 presso l'Alpe Mottac (RB);

- 1 individuo in alimentazione di notte (h. 22.00) in un prato allagato il 20/10/2001 a Cossogno (FC);

- 1 giovane e 1 adulto presso il Rifugio Pian Cavallone nel settembre 2010 (Danilo Manfrin, com. pers.);

- 1 individuo tra Provola e Finero il 17/10/2012 (BDVG);

- 1 individuo in volo al crepuscolo alle pendici del Monte Morissolino il 9/07/2014 (FC).

\section{Laridae}

\section{Zafferano (Larus fuscus) M irr}

Durante l'attività di monitoraggio della migrazione primaverile dei rapaci diurni condotta nel 2013 e 2014 sono state effettuate le seguenti osservazioni condotte dal Monte Carza:

- 1 adulto il 12/04/2013 (RB, FC);

- 6 individui diretti a $\mathrm{N}$ il 24/03/2014 (RB).

\section{COLUMBIFORMES}

\section{Columbidae}

Colombaccio (Columba palumbus) SB, M reg

Specie non comune nell'area di studio e nidificante in aree marginali. Pochi i dati noti per il periodo riproduttivo:

- 1 individuo in canto a Cossogno il 13/03/2002 e il 7/07/2003 (FC);

- 1 individuo a Miazzina il 7/07/2003 (FC);

- 1 individuo in canto presso il Monte Todum (Cossogno) il 18/05/2013 (FC);

- 1 individuo in volo territoriale presso la Madonna di Santino (S. Bernardino Verbano) il 6/03/2014 (FC);

- 1 individuo in canto sul Monte Carza il 5/05/2014 (FC, MP).

Si stima la presenza di 10-15 coppie nidificanti.

Tortora dal collare (Streptopelia decaocto) SB

Specie rara nell'area di studio per la scarsa presenza di ambienti idonei. Nidifica nel centro abitato di Vogogna (Banca dati Società di Scienze Naturali del VCO).

\section{CUCULIFORMES}

\section{Cuculidae}

Cuculo (Cuculus canorus) M reg, B

Specie comune nell'area di studio, come emerge dal dato di 38 maschi in canto che vennero censiti nel 1993 su 115 punti d'ascolto, per una dominanza relativa del 2,5\% (Movalli \& Grimaldi, 1996). Nel giugno del 1993, durante la raccolta dei dati confluiti nel sopraccitato lavoro, fu rinvenuto un nido di Codirosso spazzacamino (Phoenicurus ochruros) occupato da un giovane cuculo nei pressi del Passo dei Tre Uomini (CM).

Durante i transetti realizzati nel 2013 e 2014 nel mese di giugno per il progetto di "Monitoraggio della biodiversità" sono stati rilevati 12 maschi in canto nel 2013, mentre nel 2014 il numero è salito a 19.

Esemplari con morfismo rossiccio:

- 1 individuo il 20/05/2004 a Cossogno (FC);

- 1 individuo tra Monte Todano e Il Pizzo l'8/05/2014 (FC).

Massime densità in periodo riproduttivo:

- 4 individui in canto nell'area del Monte Carza il 5/05/2014 (FC, MP);

- 4 individui in canto tra Cappella Fina e Rifugio Pian Cavallone 1'8/05/2014 (FC).

Date precoci:

- 1 individuo in canto al Pian dei Sali (Malesco) il 10/04/2011 (FC);

- 1 individuo in canto sul Monte Carza il 17/04/2014 (FC).

Data tardiva:

- 1 maschio presso i ruderi di Vircolla (sentiero Cicogna-Pogallo) il 13/09/2014 (AM).

Si stima la presenza di 40-60 maschi cantori nell'area di studio.

\section{STRIGIFORMES}

\section{Strigidae}

Assiolo (Otus scops) M irr

L'unico dato noto di presenza nell'area di studio si riferisce a un maschio in canto nei mesi di maggio e giugno 2005 a Pollino, in comune di Premeno (AM).

\section{Gufo reale (Bubo bubo) SB}

Nell'area di studio, il gufo reale nidifica sulle pareti rocciose localizzate lungo il versante sinistro della Val d'Ossola che si affacciano sul fondovalle, sito preferenziale di alimentazione come emerso dall' analisi della dieta delle coppie nidificanti nell'area (Bionda, 2003c). Tra la fine degli anni '90 del XX secolo e l'inizio degli anni 2000 in questo settore erano presenti 4 territori. Nel decennio successivo tre di questi sono stati abbandonati ed uno solo successivamente ri-occupato. Contemporaneamente si è assistito all'insediamento di due "nuovi" territori in pareti abbandonate tra la metà degli anni ' 80 e la metà degli anni '90 del XX secolo (RB).

Alcune osservazioni:

- 1 individuo sotto l'Alpe I Curt in comune di Premosello-Chiovenda nel giugno del 2008 (AP);

- 1 individuo particolarmente debilitato, forse a causa di avvelenamento, trovato il 15/11/2013 in un giardino privato di un'abitazione di Vogogna (Elena Della Valentina, com. pers.). 
Individui non territoriali sono stati ripetutamente contattati anche in settori esterni alle aree di nidificazione (ad esempio, 1 individuo in volo presso Cicogna il 10/10/2014; AM).

Come nel resto dell'arco alpino, anche nel Verbano Cusio Ossola la specie risente di una elevata mortalità dovuta ad elettrocuzione e collisione contro cavi sospesi (Bionda, 2003c). Una seconda fonte di possibile minaccia per la specie deriva dalla pratica dell'arrampicata sportiva sulle pareti di nidificazione. Nonostante il gufo reale si dimostri più tollerante alla vicinanza dell'uomo del falco pellegrino, il disturbo arrecato durante la cova o le prime fasi dell'allevamento dei giovani determina generalmente l'abbandono della covata (Bionda, 2003c). Un'altra importante minaccia per questa specie nel Verbano Cusio Ossola è costituita dalle modificazioni ambientali legate all'espansione della superficie urbanizzata a scapito degli ambienti aperti ed alla scomparsa/artificializzazione dei corsi d'acqua, fattori in grado di influenzare negativamente il successo riproduttivo (Bionda \& Brambilla, 2012).

\section{Civetta nana (Glaucidium passerinum) M irr}

Nel 2012, la presenza della specie è stata rilevata per la prima volta nell'area di studio. Il 30/03/2012 è stato infatti udito per parecchi minuti il canto non indotto da playback di un maschio in località Pizzo Faiè, a 1.100 metri di altitudine (FC \& Andrew Cottam in Casale, 2014). Si tratta di un'osservazione al di fuori dell'areale noto della specie. Nel Verbano Cusio Ossola sono noti almeno tre casi di osservazioni in situazioni al di fuori dell'areale tipico, ovvero 2 individui in castagneti a $900 \mathrm{~m}$ s.l.m. e 1 individuo in canto il 13/03/2002 in un rimboschimento di abete rosso sul Mottarone, ascrivibili a giovani in dispersione (RB).

Al fine di verificare l'eventuale presenza regolare della specie all'interno dell'area protetta, nel corso delle stagioni riproduttive 2013 e 2014 la civetta nana è stata oggetto di specifica indagine, tramite utilizzo di playback, all'interno dei confini del Parco e in aree ad esso limitrofe potenzialmente idonee per la specie, rientranti nell'area di studio. In particolare, sono state indagate le peccete mature risultanti da rimboschimenti e le abetine. La presenza della specie non è stata tuttavia accertata. È noto, d'altro canto, che in caso di presenza di maschi tra loro isolati, il tempo di latenza della risposta al playback si protrae moltissimo nel tempo (anche di ore) ed è quindi non corretto considerare assente la specie solo in quanto non si manifesta nei tempi di ascolto solitamente impiegati in questo tipo di censimenti (Borgo \& Genero, 2011).

\section{Civetta (Athene noctua) SB}

Sono noti pochissimi dati di presenza relativi alla fascia collinare nel settore meridionale dell'area di studio:

- nidificazione accertata a Bracchio di Mergozzo nel 1997 (Roberto Molinari, com. pers.);

- 1 individuo in canto a Bée nel 1997 (LP) e ancora nel 2001 (FC).

Si stima la presenza di 1-3 coppie nell'area di studio.

\section{Allocco (Strix aluco) SB}

Specie ampiamente diffusa nell'area di studio. L'attività di monitoraggio di Civetta nana (Glaucidium passerinum), Civetta capogrosso (Aegolius funereus) e Succiacapre (Caprimulgus europaeus), condotta nel 2013 e 2014, ha portato a individuare anche 24 territori di allocco tramite emissione di canto spontaneo (senza stimolazione acustica). L'allocco è stato udito in canto spontaneo anche in pieno giorno, ad esempio il 20/03/2014 tra Cappella Porta e Cappella Fina (FC, LL). Si segnala altresì la presenza di 1 adulto e 2 giovani presso Creves (Cursolo-Orasso) 1'11/07/2014 (FC, CM, AP).

Si stima la presenza di 40-50 coppie nidificanti nell'area di studio.

\section{Gufo comune (Asio otus) SB}

Specie elusiva, per la quale sono noti pochi dati di presenza nell'area di studio. Nel 2013 è stata rilevata la sua presenza in periodo riproduttivo tra Alpe Pala e Cappella Fina (Miazzina), con un individuo osservato il 2/04/2013 a seguito di playback (FC). Sono inoltre noti i seguenti dati:

- 1 giovane predato a Colloro il 6/05/2010 (BDVG);

- resti di predazione di 1 individuo (spiumata) all'Alpe Vald di Sopra il 5/09/2010 (BDVG).

\section{Civetta capogrosso (Aegolius funereus) SB}

Gli studi preliminari alla stesura del Piano del Parco (EPNVG, 1998) indicavano la civetta capogrosso come una specie per la quale non erano noti dati di presenza. Occorre d'altro canto rilevare che in quegli anni lo stesso Picchio nero (Dryocopus martius) veniva ritenuto dagli estensori del Piano del Parco "particolarmente scarso" non avendo "raccolte informazioni comprovanti una presenza come nidificante all'interno degli attuali confini del Parco" (EPNVG, 1998: pag. 180). Durante gli studi preliminari alla stesura del Piano, compiuti nel 1997, la specie non venne infatti mai contattata tramite indagini condotte con utilizzo di playback che compresero: 234 $\mathrm{km}$ in auto lungo strade nelle valli Grande, Intrasca, Cannobina, Vigezzo e d'Ossola, $58 \mathrm{~km}$ di itinerari a piedi e 15 punti di osservazione. L'Atlante degli uccelli nidificanti del Verbano Cusio Ossola, che comprende dati raccolti tra il 1996 e il 2005, cita un dato di nidificazione possibile in una località a nord-est del Parco, in Comune di Malesco (Bionda \& Bordignon, 2006). La specie risulta ben distribuita nelle valli del Canton Ticino limitrofe al confine nord-orientale dell'area di studio (Antoniazza, 2013).

$\mathrm{Nel}$ corso delle stagioni riproduttive 2013-2014, la specie è stata oggetto di specifica indagine all'interno dei confini del Parco e in aree ad esso limitrofe ritenute vocate per la specie (Casale, 2014). Tale indagine ha permesso di accertare per la prima volta la presenza della specie nel Parco e di accertarne la nidificazione. In particolare, la presenza della civetta capogrosso è stata riscontrata in tre siti all'interno dei confini del Parco, uno in Val Grande e due in Val Pogallo, ovvero:

- 1 giovane 1'11/06/2013 in Val Portaiola, Val Grande (FC, CM, PD);

- 1 adulto in canto il 18/06/2013 in Val Pogallo (FC, $\mathrm{CM}$; 
- 1 giovane il 2/07/2013 in Val Pogallo (LL).

I tre siti in cui la specie è stata rilevata sono localizzati in corrispondenza di boschi di faggio con sviluppo colonnare del tronco, con individui di grandi dimensioni (numerosi con diametro maggiore di $40 \mathrm{~cm}$ a $1,30 \mathrm{~m}$ di altezza) e con presenza di picchio nero (udito o osservati segni di alimentazione o nidi). I due siti localizzati in Val Pogallo distano tra loro $2,3 \mathrm{~km}$, mentre distano rispettivamente 7 e $9 \mathrm{~km}$ rispetto al sito in Val Grande. La bassa densità rilevata è ascrivibile a:

- un probabile recente insediamento della specie nel Parco, a seguito dell'insediamento del picchio nero con un numero significativo di coppie nidificanti;

- una scarsa risposta al richiamo da parte dei maschi in presenza di densità basse. La maggior densità della specie garantisce infatti una più pronta risposta alla stimolazione acustica (playback), mentre in presenza di maschi isolati il tempo di latenza della risposta varia moltissimo ed è spesso imprudente considerare assente la specie solo perché non si manifesta una risposta nei tempi di ascolto solitamente impiegati per questo tipo di censimento (Borgo \& Genero, 2011);

- un'elevata densità di allocco in vasti settori del Parco che influenza negativamente la presenza della civetta capogrosso. Infatti, le due specie possono competere per la cavità di nidificazione e probabilmente anche per le risorse alimentari. La competizione può talora sfociare nella predazione della civetta capogrosso da parte dell'allocco. In zone di simpatria è dunque verosimile che, per ridurre i rischi di predazione da parte dell'allocco, la civetta capogrosso deprima la sua attività di canto notturna, che la esporrebbe a una più facile localizzazione, supplendo a tale diminuzione con una maggiore attività di canto diurna (Borgo, 1999). Il canto rilevato durante il monitoraggio svolto nel 2013 è stato effettivamente udito di giorno, alle ore 8.45 .

\section{CAPRIMULGIFORMES}

\section{Caprimulgidae}

Succiacapre (Caprimulgus europaeus) M reg, B

Nel Parco la specie nidifica soprattutto nel settore meridionale, lungo il versante esposto a Sud compreso tra Vogogna e Cuzzago (Bionda \& Bordignon, 2006; Casale \& Brambilla, 2010b). I dati pregressi permettono di definire il seguente quadro di presenze:

- 1 maschio territoriale in canto il 13/06/1999 presso il bacino sopra Colloro (CM);

- 1 maschio territoriale in canto il 21/06/2001 sul Monte Carza (CM);

- 2-6 maschi territoriali nel periodo 2004-2006 nei boschi termofili di versante nei pressi dell'abitato di Vogogna (Bionda \& Bordignon, 2006);

- 4-5 maschi territoriali nei pressi dell'Alpe Lut, sopra Colloro (Casale \& Brambilla, 2010b; Fig. 18);

- 1 maschio territoriale nei pressi di Rovegro nel 2009 (ST);
- 1 maschio territoriale a Cosasca nel 2010 (RB);

- 2 maschi territoriali a Nibbio nel 2010 (RB);

- 2 maschi territoriali presso l'abitato di Colloro nel 2012 (MD).

Attività di monitoraggio della specie sono state condotte nel corso delle stagioni riproduttive 2013 e 2014 nelle aree maggiormente vocate alla sua presenza nell'area di studio. I risultati emersi sono presentati in Tab. 1.

La distanza minima tra maschi in canto contemporaneo è stata di 500 metri, rilevata in località Alpe Lut. In base ai dati disponibili, è stimabile che la popolazione nidificante di succiacapre compresa nell'area di studio sia di 14-24 maschi territoriali così suddivisi (Fig. 19):

- 8-16 nel Parco;

- 6-8 nell'area di studio esterna al Parco.

\section{APODIFORMES}

\section{Apodidae}

Rondone comune (Apus apus) M reg, B, Err

La specie nidifica in alcuni centri abitati compresi nell'area di studio (ad esempio, Vogogna, Aurano, Miazzina) e inoltre frequenta le aree montane, in particolare le dorsali caratterizzate da praterie, con gruppi spesso numerosi per lo svolgimento di attività trofica; ad esempio:

- oltre 200 individui sopra Cossogno il 14/07/2001 (FC);

- circa 250 individui presso il Monte Carza il 17/05/2014 e circa 100 individui il 24/05/2014 (FC, MP);

- numerose decine di individui osservati in più occasioni tra il 2009 e il 2014 sorvolare la dorsale tra Passo Folungo e il Monte Zeda e la dorsale tra Pizzo Pernice e Monte Todano, spesso frammisti a gruppi di Rondone maggiore (Apus melba) (FC).

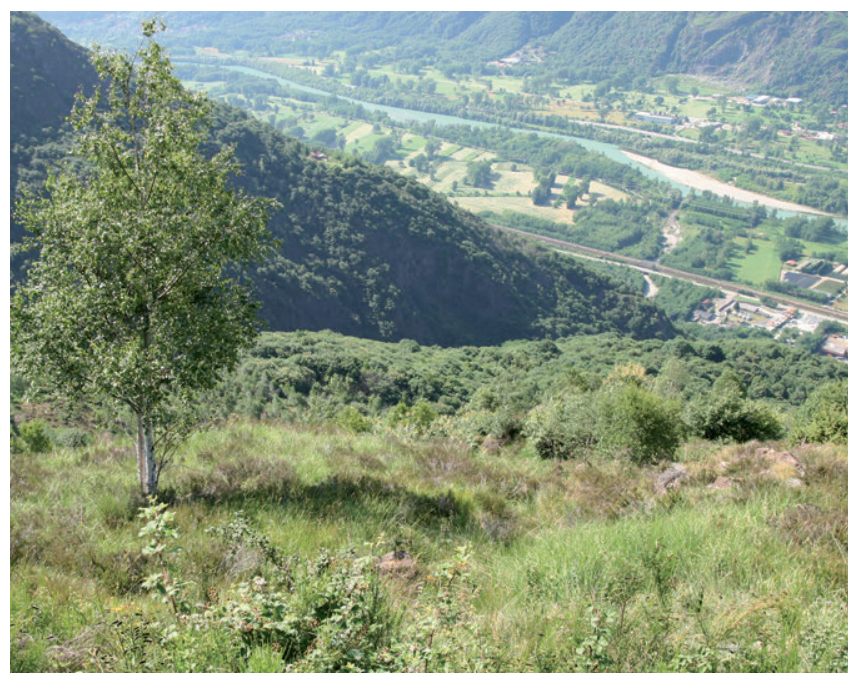

Fig. 18 - Brughiera a Calluna vulgaris lungo il versante meridionale del Parco, habitat idoneo per il succiacapre. / Calluna vulgaris heathland along the Southern side of the Park, a suitable habitat for Nightjar. (Foto / Photo Fabio Casale). 
Rondone pallido (Apus pallidus) M irr

La specie nidifica nei vicini centri abitati di Cannobio e Domodossola (Bionda \& Bordignon, 2006). Nell'area di studio sono note le seguenti segnalazioni: 2 individui il 9/09/2003 nei pressi di Cossogno (Casale \& Brambilla, 2010b); 2 individui in località i Balmitt il 2/05/2009 (RB); almeno un individuo udito il 22/07/2009 a Passo Folungo, frammisto a numerosi rondoni comuni e rondoni maggiori (Casale \& Brambilla, 2010b).

Rondone maggiore (Apus melba) $\mathrm{M}$ reg, B, Err La nidificazione della specie nell'area di studio è

Tab. 1 - Territori di Succiacapre rilevati nel Parco e aree limitrofe nel 2013 e 2014. / Nightjar territories recorded in the Park and surrounding areas in 2013 and 2014.

\begin{tabular}{|c|c|c|c|c|}
\hline Area campione & Comuni & $\begin{array}{l}\text { Numero } \\
\text { territori }\end{array}$ & Parco & $\begin{array}{c}\text { Area esterna al Parco, } \\
\text { compresa nell'area } \\
\text { di studio }\end{array}$ \\
\hline Versante Vogogna - Premosello & Vogogna, Premosello-Chiovenda & 2 & + & \\
\hline Alpe Lut & Premosello-Chiovenda & $4-6$ & + & \\
\hline Dorsale da Alpe Ompio a Mergozzo & San Bernardino Verbano, Bieno, Mergozzo & 3 & & + \\
\hline Biogna & Aurano & 2 & + & \\
\hline Monte Morissolino & Oggebbio & 1 & 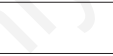 & + \\
\hline Monte Carza & Trarego-Viggiona & 1 & & + \\
\hline Totale territori censiti & & 13-15 & 8-10 & 5 \\
\hline
\end{tabular}

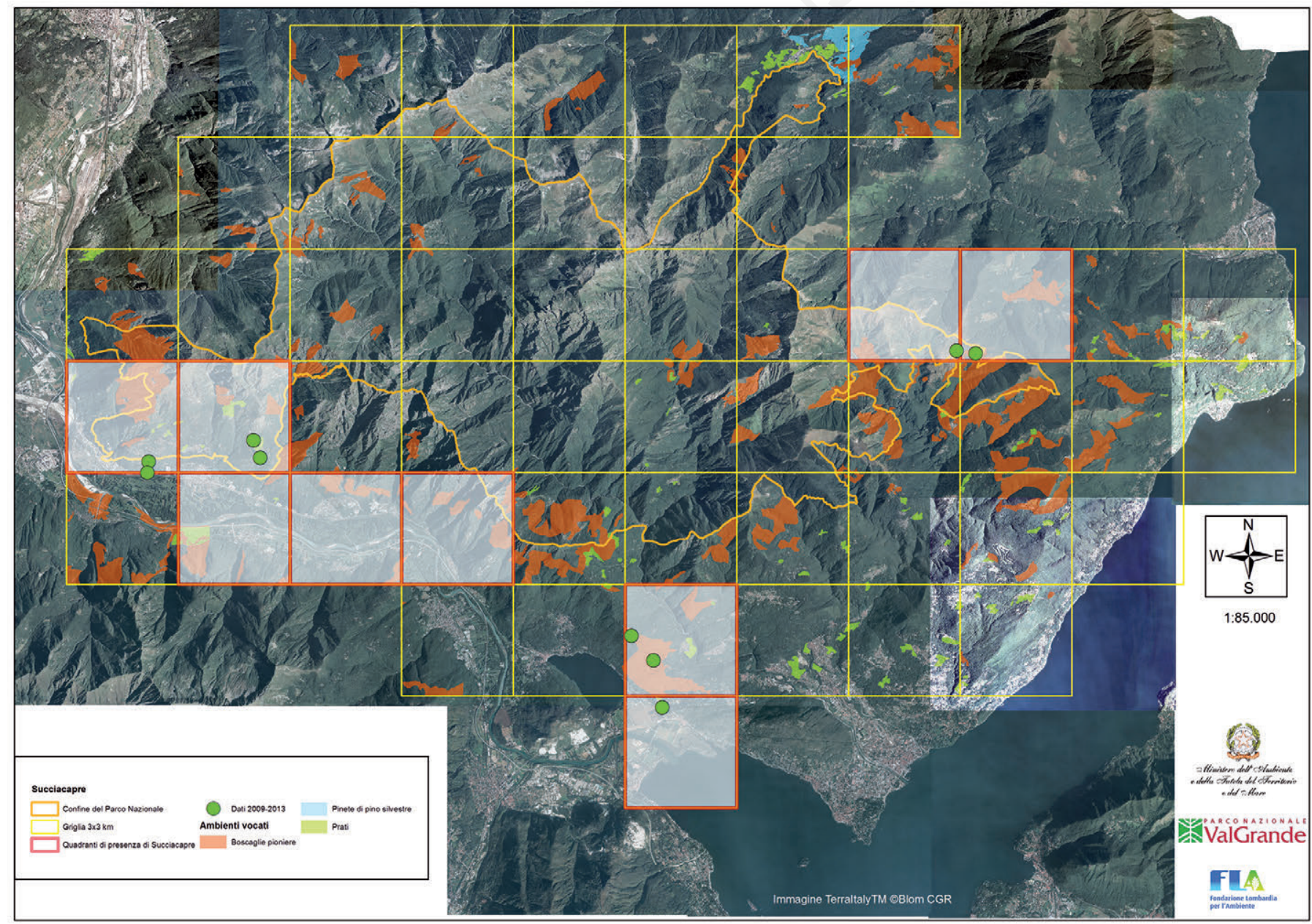

Fig. 19 - Succiacapre nel Parco Nazionale della Val Grande e aree limitrofe. Quadranti colorati: aree in cui la specie è stata rilevata in periodo riproduttivo nel 2009-2013; pallini verdi: siti di presenza rilevati tramite attività di monitoraggio nel 2013. / Nightjar in Val Grande National Park and surrounding areas. Colored squares: areas were the species was recorded in 2009-2013 during the breeding season; green dots: territories recorded in 2013. 
stata accertata nei centri abitati di Gurro e Premeno, ma anche in corrispondenza di ambienti naturali (Bionda \& Bordignon, 2006). Frequenta le aree montane con gruppi spesso numerosi (fino a 50 individui), soprattutto lungo le dorsali con ambienti aperti (ad esempio, tra Passo Folungo e Monte Zeda e tra Pizzo Pernice e Monte Todano) per lo svolgimento dell'attività trofica, spesso frammisto ai rondoni comuni. Data più precoce: 8 individui al Pian dei Sali (Malesco) il 10/04/2011 (FC).

\section{CORACIIFORMES}

\section{Meropidae}

\section{Gruccione (Merops apiaster) M irr}

In periodo autunnale, negli ultimi anni, si segnala un significativo flusso migratorio lungo $i$ versanti sud-occidentali del Parco, nei comuni di Vogogna e Premosello-Chiovenda, con numerose decine di individui osservati a valle di Genestredo, di Colloro e tra Premosello e Cuzzago nel 2013 e nel 2014, tra la metà di agosto e la metà di settembre (BDVG e CM). Altrove vi è una sola osservazione nota: uno stormo di circa 30 individui in migrazione primaverile sopra Cappella Fina (Miazzina) il 12/05/2013 (Giovanni Natale \& Maria Grazia Carpi nella banca dati on-line Ornitho.it).

\section{Upupidae}

Upupa (Upupa epops) M irr

Specie scarsamente rilevata nell'area di studio:

- 1 individuo il 5/04/2001 a Dorbolo, presso Finero (RB);

- 1 individuo osservato a Pollino per due giorni consecutivi nel maggio 2012 (AM).

\section{PICIFORMES}

\section{Picidae}

Torcicollo (Jynx torquilla) M reg, B

Specie scarsamente rilevata nell'area di studio. Un solo territorio (rilevato a Cicogna, loc. Merina, il 24/05/1993) era stato individuato nel Parco da Movalli \& Grimaldi (1996) su 115 punti d'ascolto. Successivamente si segnalano: un giovane da poco involato il 26/06/1996 poco sotto l'Alpe I Curt di Colloro (CM), 1 individuo il 5/07/2000 vicino all'Alpe Colle (CM) e un dato di nidificazione possibile viene segnalato da Bionda \& Bordignon (2006) in Valle Intrasca.

\section{Picchio verde (Picus viridis) SB}

Specie non comune nell'area di studio, legata soprattutto a margini boscati e ad aree prative con boschetti ed alberi isolati, per lo più localizzate nei pressi di villaggi. 6 territori sono stati individuati nel Parco nel 1993 (Movalli \& Grimaldi, 1996) su 115 punti d'ascolto. Alcuni dati in periodo riproduttivo:
- 1 individuo in canto nei pressi di Corte Buè il 19/04/1997 (CM);

- 1 individuo in canto sopra Cossogno in località La Cresta il 4/06/1998 (CM);

- 1 individuo in canto il 16/04/2000 presso Aurano;

- 1 individuo a Intragna il 18/05/2000 (FC);

- 1 individuo il 19/03/2002 alle pendici del Monterosso (Verbania: FC);

- 1 individuo in canto il 24/03/2002 tra Ungiasca e Cossogno (FC);

- 1 individuo in canto il 12/05/2002 a Cappella Porta (CM);

- 1 individuo in canto il 15/05/2002 presso l'Alpe Caseracce (CM);

- 1 individuo il 7/07/2003 presso Miazzina (FC);

- 1 individuo a Cicogna il 18/03/2010 e il 14/07/2010 (BDVG);

- 1 individuo a Ponte Casletto il 4/03/2011 e il 23/05/2012 (BDVG);

- 1 individuo il 28/05/2011 tra Cicogna e Pogallo (BDVG);

- 1 individuo in canto il 6/04/2013 alle pendici della Cima di Morissolo (RB, FC, CM, FCL, DF, PP, LP);

- 1 individuo l'11/04/2013 presso l'Alpe Pianezza di Vogogna (BDVG);

- 1 individuo all'Alpe Ompio il 24/04/2013 (FC, LL, $\mathrm{CM}$ ).

Si stima la presenza nell'area di studio di 15-20 coppie nidificanti.

\section{Picchio nero (Dryocopus martius) SB}

Negli studi preliminari alla stesura del Piano del Parco (metà anni '90 del XX secolo) il picchio nero veniva ritenuto dagli estensori "particolarmente scarso" non avendo "raccolte informazioni comprovanti una presenza come nidificante all'interno degli attuali confini del Parco" (EPNVG, 1998: pag. 180); durante gli studi preliminari alla stesura del Piano, compiuti nel 1997, la specie non venne infatti mai contattata tramite indagini che compresero: $234 \mathrm{~km}$ in auto lungo strade nelle valli Grande, Intrasca, Cannobina, Vigezzo e d'Ossola, $58 \mathrm{~km}$ di itinerari a piedi e 15 punti di osservazione. L'insediamento della specie nell'area protetta dovette comunque compiersi in quel periodo, in quanto Movalli \& Grimaldi (1996), a seguito di un'indagine condotta nel 1993 tramite punti d'ascolto, riferiscono la presenza di 4 territori di tale specie e un grosso faggio con alcune cavità nido fu trovato nei pressi dell'Alpe Basciot (CM).

Nel 2010 la popolazione viene stimata in 5-10 coppie sulla base di dati occasionali e senza avere svolto specifiche indagini (Casale \& Brambilla, 2010a).

Nel corso della stagione riproduttiva 2013 è stata monitorata la presenza della specie all'interno dell'area di studio (Casale, 2014). L'indagine è stata condotta nelle principali aree vocate alla sua presenza (habitat forestali maturi). I dati raccolti nel 2013, uniti a quelli pregressi relativi agli ultimi anni (2009-2012), permettono di valutare che la specie presenta una distribuzione sostanzialmente uniforme in tutti gli habitat forestali (in particolare faggete, castagneti, peccete) all'interno dell'area di studio, ad eccezione di alcuni tratti di boschi 
cedui non sufficientemente evoluti (ad esempio, la valle tra Colloro e la Colma di Premosello, ove la specie è comunque regolarmente presente al di fuori del periodo riproduttivo) (Fig. 20). La popolazione locale assomma attualmente ad almeno 20-23 territori, così suddivisi:

- 11-13 all'interno dei confini del Parco Nazionale;

- 9-10 in aree limitrofe al Parco (comprese nell'area di studio).

Nella Tab. 2 vengono fornite indicazioni sulla distribuzione dei territori rilevati nel periodo 2009-2014.

In base alla disponibilità di aree vocate alla presenza della specie, ma non indagate in quanto in buona parte non accessibili, è verosimile stimare una popolazione nidificante all'interno dei confini del Parco di 15-20 coppie, e di circa 30 coppie considerando l'intera area di studio.

Tab. 2 - Territori di picchio nero nel Parco e aree limitrofe nel 20092014. / Black Woodpecker territories recorded in the Park and surrounding areas in 2009-2014.

\begin{tabular}{|l|c|}
\hline \multicolumn{2}{|c|}{ Territori entro i confini del Parco Nazionale } \\
\hline Località & Territori \\
\hline Valle di Finero & $1-2$ \\
\hline Alpe Vald di Sopra & 1 \\
\hline Alpe Mottac & 1 \\
\hline Val Portaiola & 1 \\
\hline Val Serena - Val Gabbio & $1-2$ \\
\hline Colloro & 1 \\
\hline Cicogna & 1 \\
\hline Pogallo & 1 \\
\hline Media e alta Val Pogallo & $1-2$ \\
\hline Corte Buè & 1 \\
\hline Cappella Porta - Rifugio Pian Cavallone & 2 \\
\hline Totale territori entro i confini del Parco & $12-15$ \\
\hline \multicolumn{1}{|c|}{ Territori limitrofi al Parco Nazionale } \\
\hline Località & \\
\hline Media e alta Val Loana & Territori \\
\hline Valle del Basso & $1-2$ \\
\hline Alpe Parpinasca & 1 \\
\hline Alpe Ogliana & 1 \\
\hline Alpe Ompio & 1 \\
\hline Cappella Fina (Miazzina) & 1 \\
\hline Cima di Morissolo & 1 \\
\hline Istituto Auxologico di Piancavallo & 1 \\
\hline Monte Bavarione & $\mathbf{1 1 - 1 2}$ \\
\hline Monte Carza - versante Valle Cannobina \\
\hline Valle del Rio Cannero & 1 \\
\hline Totale territori in aree limitrofe al Parco & \\
\hline Totale territori nell'area di studio & \\
\hline & \\
\hline
\end{tabular}

Nel 2013 sono stati altresì rinvenuti e georeferenziati 2 alberi-nido, in particolare due faggi vivi con fusto colonnare e liscio ed inserzione della chioma piuttosto alta, alle quote di 1.057 e $972 \mathrm{~m}$ s.l.m.. Sono stati inoltre rilevati 37 alberi con cavità realizzate dal picchio nero per attività di foraggiamento. Tali alberi appartenevano alle specie indicate in Tab. 3. La specie prevalente è risultata il faggio ( $38 \%$ dei casi).

\section{Picchio rosso maggiore (Dendrocopos major) SB}

Specie apparentemente meno comune nell'area di studio di quanto ci si possa aspettare. Movalli \& Grimaldi (1996), a seguito di un'indagine condotta tramite 115 punti d'ascolto nel 1993, non citano la specie tra quelle rilevate nell'area protetta e nel 2013-2014, nell'ambito del progetto di Monitoraggio della biodiversità (Casale 2014; LL, MP), sono stati individuati in media 3 territori su 24 punti d'ascolto. Una possibile spiegazione consiste nel fatto che l'area di studio risulta in gran parte inaccessibile a causa dell'innevamento nel periodo di massima contattabilità della specie (inizio primavera). Nel periodo 2010-2014, i dati raccolti dal CFS lo hanno segnalato in periodo riproduttivo nelle seguenti località comprese nel Parco, ma non interessate dai punti d'ascolto del 2013 e 2014: Cicogna, Corte Borlino, Alpe Ompio, Ponte Cadenesc, Capraga, Uccigiola, Crosane, Alpe Velina di Sotto, Alpe Pianoni, Alpe Monticello (BDVG).

Si stima la presenza di 40-50 coppie nidificanti nell'area di studio.

\section{Picchio rosso minore (Dendrocopos minor) SB}

Scarsi i dati di presenza della specie nell'area di studio, qui di seguito elencati:

- Cossogno: 1 individuo il 5/05/2001 in un castagneto nei pressi del paese (FC);

- Val Pogallo: 1 individuo il 5/07/2014 presso Pogallo (LL, MP), ove la specie era già stata in precedenza rilevata a inizio anni 2000 (MD); maschio in canto il $14 / 4 / 1997$ e il $24 / 3 / 2001$ tra Cicogna e Pogallo (RB);

- Valle di Finero: una coppia (1 individuo con

Tab. 3 - Alberi con presenza di segni di attività di foraggiamento di picchio nero. / Trees with signs of feeding activity by Black Woodpecker.

\begin{tabular}{|l|c|c|}
\hline Specie & Numero individui & $\mathbf{\%}$ \\
\hline Fagus sylvatica & 14 & 38 \\
\hline Alnus incana & 5 & 13 \\
\hline Picea abies & 4 & 11 \\
\hline Betula pendula & 4 & 11 \\
\hline Salix sp. & 3 & 9 \\
\hline Fraxinus excelsior & 2 & 5 \\
\hline Prunus avium & 2 & 5 \\
\hline Castanea sativa & 2 & 5 \\
\hline Populus tremula & 1 & 3 \\
\hline Totale individui & $\mathbf{3 7}$ & $\mathbf{1 0 0}$ \\
\hline
\end{tabular}


imbeccata) 1'1/07/2009 (Casale \& Brambilla, 2010b).

Si stima la presenza di 5-10 coppie nell'area di studio.

\section{PASSERIFORMES}

\section{Alaudidae}

Tottavilla (Lullula arborea) M irr

La presenza della specie è stata riscontrata solo durante la migrazione autunnale:

- il 10/10/2010 8 individui a Pian Vadà (FC);

- il 30/10/2013 1 individuo presso la Bocchetta di Cavalla (FC, DR).

Allodola (Alauda arvensis) M reg, B

Nel Parco, Movalli \& Grimaldi (1996), nell'indagine condotta tramite punti d'ascolto nel 1993, non citano la specie tra quelle rilevate nell'area protetta, verosimilmente in quanto la principale area di presenza dell'allodola (settore orientale del Parco) era all'epoca esterna ai confini del Parco e vi è stata solo successivamente inserita a seguito di ampliamento. Negli anni successivi furono rilevati 2 individui in canto sul Monte Morissolino il 19/06/2001 (CM) e 1 individuo in canto a Obogno presso il Monte Carza il 22/06/2001 (CM).

L'area di recente presenza della specie comprende le dorsali Passo Folungo-Pian Vadà e Monte Todano-Il Pizzo, caratterizzate da vasti versanti erbosi e ben soleggiati (Fig. 21). Nel primo sito sono stati rilevati: 1 territorio il 22/06/2002, 2 territori il 21 e 29/06/2012, 1 territorio il 25/06/2013 (FC). Nel secondo sito sono stati rilevati 3 territori $1^{\prime} 8 / 05 / 2013$ e 1 territorio $1^{\prime} 8 / 05 / 2014$ (FC). Si segnalano situazioni di estinzione locale, in quanto nel 2010-2014 non è più stata confermata la presenza della specie in periodo riproduttivo sui monti Morissolino e Carza.

La popolazione nidificante nell'area di studio è stimabile in 3-7 coppie.

In migrazione:

- stormo di 25 individui osservato il 6/10/2007 da Scaredi risalire la Val Loana e entrare in Val Grande, diretto verso S (FC);

- 2 individui in sorvolo il 25/10/2013 alla Colma di Premosello (FC, CM, MP, MD);

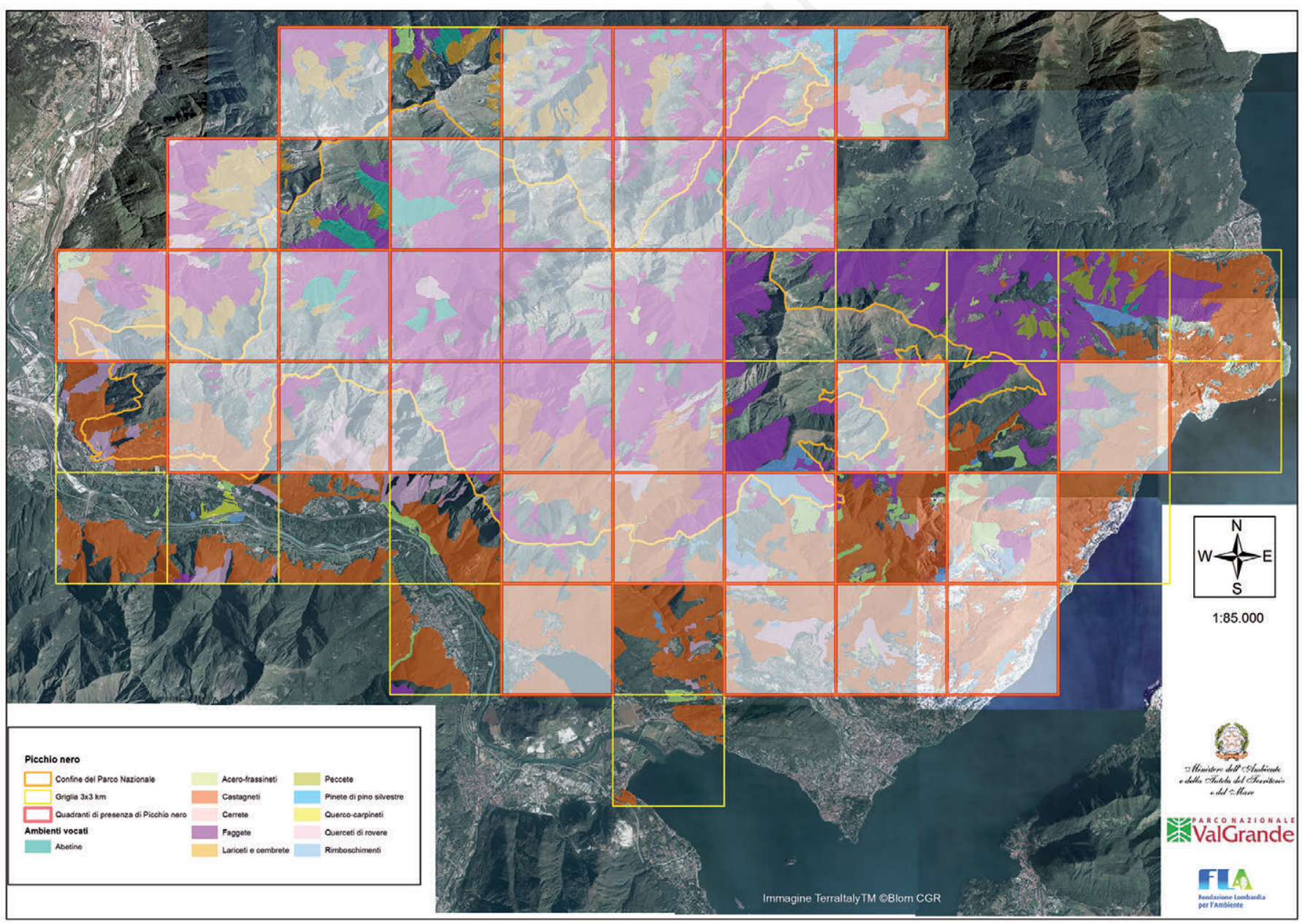

Fig. 20 - Picchio nero nel Parco Nazionale della Val Grande e aree limitrofe (dati 2009-2013). I quadranti colorati indicano aree in cui la specie è stata rilevata in periodo riproduttivo. / Black Woodpecker in Val Grande National Park and surrounding areas (data 2009-2013). Colored squares indicate were the species was recorded during the breeding season. 
- 1 individuo in migrazione il 30/10/2013 alla Bocchetta di Cavalla (FC, DR);

- 1 individuo diretto a N il 17/05/2014 sul Monte Carza (FC, MP).

\section{Hirundinidae}

Rondine montana (Ptyonoprogne rupestris) M reg, B

Specie piuttosto diffusa ma non comune come nidificante nell'area di studio, con l'eccezione della Val Cannobina dove è presente con alcune piccole colonie in corrispondenza dei ponti sul Rio Cannobino. La sua presenza non era stata rilevata nell'ambito dell'indagine condotta nel 1993 (Movalli \& Grimaldi, 1996). È stata occasionalmente segnalata come svernante lungo le rive del vicino Lago Maggiore (Bionda \& Bordignon, 2006).

In periodo riproduttivo:

- alcuni individui nei pressi di pareti rocciose lungo il sentiero tra Colloro e Genestredo, con almeno un nido occupato, 1'8/05/11 (CM, AP);

- una coppia in Val Pogallo, presso il Rio Caslù, il 17/06/2013 (FC, CM);

- 1 individuo alla Bocchetta di Terza, in alta Val Pogallo, il 17/06/2013 (FC, CM, ST);
- una coppia presso Ponte Casletto il 24/04/2014 (BDVG);

- 6 individui tra Lut e Alpe La Piana il 31/05/2014 (LL, MP);

- almeno 5 individui presso il ponte per Cavaglio sul Rio Cannobino il 5/06/2014 (FC);

- 5 individui presso il ponte sul Rio Cannobino sovrastante l'Orrido di Sant'Anna il 7/06/2014 (CM);

- 1 individuo presso il Passo Folungo (Aurano) il 15/06/2014 (FC);

- 4 individui a Pogallo il 17/06/2014 (LL, MP).

In migrazione:

- 2 individui diretti a $\mathrm{N}$ alla Cresta della Ciresa il 6/04/2013 (RB, FC, CM, FCL, DF, PP, LP);

- 1 individuo diretto a $\mathrm{N}$ dal Monte Carza il 12/04/2013 (RB, FC);

- 10 individui diretti a S a Scaredi il 18/10/2014 (FC, MP).

Nell'area di studio si stima la presenza di 30-50 coppie.

Rondine (Hirundo rustica) MB

Nidifica con poche coppie in alcuni centri abitati compresi nell'area di studio. Nessuna coppia individuata

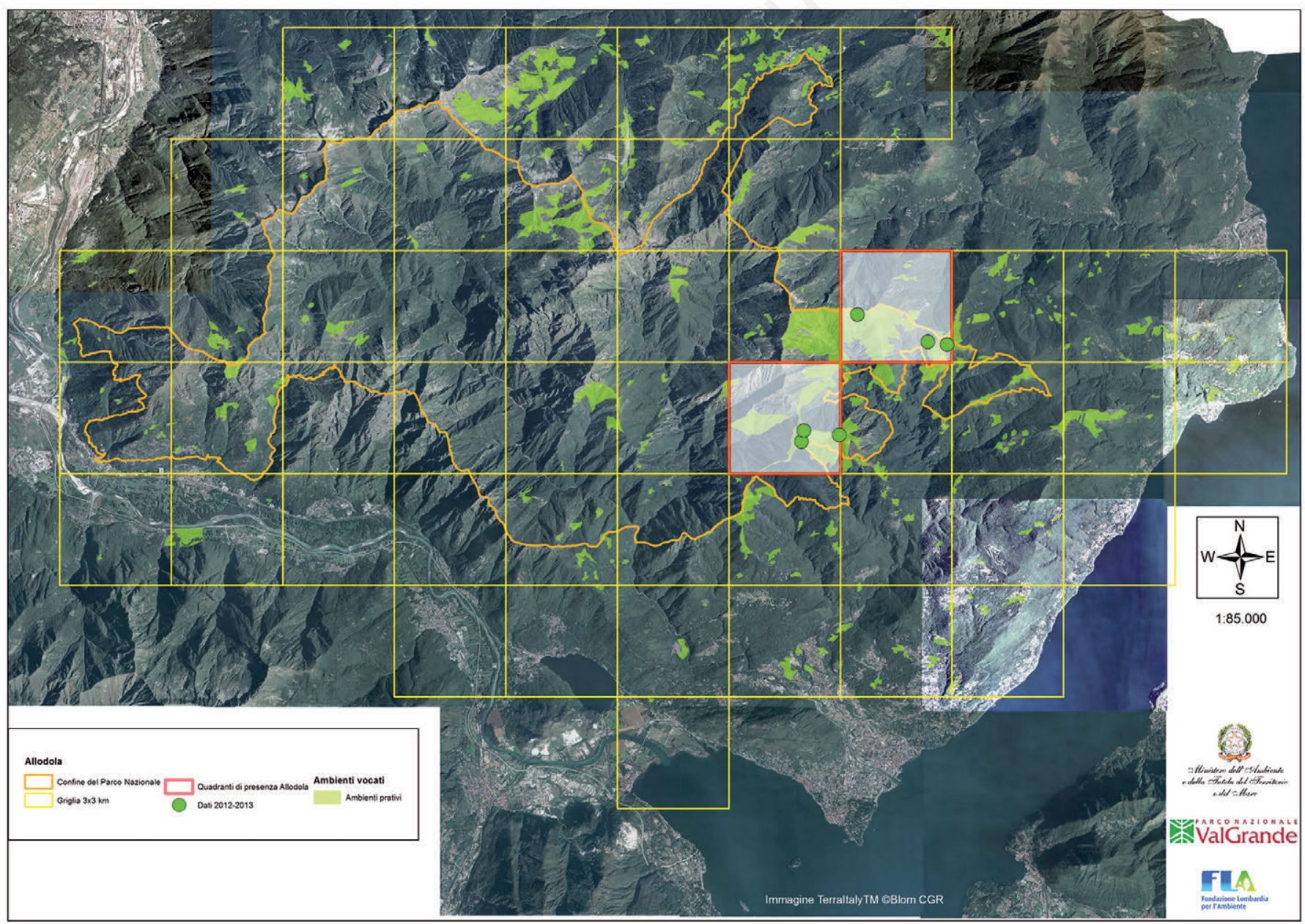

Fig. 21 - Allodola nel Parco Nazionale della Val Grande e aree limitrofe. Quadranti colorati: aree in cui la specie è stata rilevata in periodo riproduttivo nel 2009-2014; pallini verdi: dati di presenza in periodo riproduttivo nel 2012-2014. / Skylark in Val Grande National Park and surrounding areas. Colored squares indicate were the species was recorded during the breeding season in 2009-2014 and green dots in 2012-2014. 
nel Parco a metà anni '90 (Movalli \& Grimaldi, 1996) su 115 punti d'ascolto. Alcuni dati di nidificazione: un nido occupato il 17/07/2001 a Cossogno (FC), una coppia a Finero 1'1/07/2009 (FC).

In migrazione primaverile:

- dal Monte Carza: 62 individui il 12/04/2013 (RB, FC), 6 individui il 17/04/2014 (FC, CM), 15 individui il 5/05/2014 (FC, MP), 29 il 17/05/2014 (FC, MP);

- sulla dorsale Pizzo Pernice-Monte Todano: 24 individui l'8/05/2014 (FC).

In migrazione autunnale: 5 individui a Scaredi 1'8/09/2014 (FC, MP).

\section{Balestruccio (Delichon urbicum) MB}

Nidifica con poche coppie in alcuni centri abitati compresi nell'area di studio. Alcuni dati di nidificazione:

- un nido occupato il 12/07/2001 a Bieno (FC);

- almeno un nido attivo il $25 / 06 / 2013$ presso l'Istituto Auxologico di Piancavallo (FC).

In migrazione:

- circa 100 individui presso 1'Alpe Erta (Valle del Basso, Malesco) il 13/09/2013 (FC, CM, MP);

- 3 individui sopra il Monte Carza diretti a $\mathrm{N}$ il 12/04/2013 (RB, FC);

- 2 individui sopra il Monte Carza diretti a $\mathrm{N}$ il 17/04/2014 (FC, CM) e uno il 5/05/2014 (FC, MP);

- 21 individui in sorvolo verso $\mathrm{N}$ sulla dorsale tra Pizzo Pernice e Monte Todano l'8/05/2014 (FC);

- circa 400 individui a Scaredi 1'8/09/2014 (FC, MP).

\section{Motacillidae}

Calandro (Anthus campestris) M reg

La specie viene avvistata con relativa regolarità durante le migrazioni, ma con pochi individui:

- 7 individui a Pian d'Arla nel settembre 1999 (RB, LP);

- maschio in canto sulla Cima di Morissolo il 9/05/2000 (RB);

- 1 individuo alla Colma di Basagrana il 28/08/2012, frammisto a circa 15 spioncelli e un Prispolone (Anthus trivialis) (FC e AG);

- 1 individuo in sorvolo, diretto a S, nella Valle del Basso (Malesco) il 13/09/2013 (FC, CM, MP);

- 1 individuo in sorvolo sul Monte Carza, diretto a $\mathrm{N}$, il 5/05/14 (FC, MP) e 1 individuo il 24/05/14 (FC, MP).

\section{Prispolone (Anthus trivialis) M reg, B}

Specie comune come nidificante negli ambienti adatti, in particolare nella fascia altitudinale intorno ai $1.500 \mathrm{~m}$ s.l.m., dove crescono ancora alberi isolati tra la vegetazione erbacea e arbustiva dominante. 45 territori individuati nel Parco nel 1993 su 115 punti d'ascolto per una dominanza relativa del 2,9\% (Movalli \& Grimaldi, 1996).

Alcuni dati in periodo riproduttivo:

- 3 individui in canto nella Valle di Finero 1'1/07/2009, per una densità di 2,5 territori $/ \mathrm{km}$ (Casale \& Brambilla, 2010b);
- 8 individui in canto lungo il percorso Rifugio Pian Cavallone-Monte Todano-Il Pizzo l'8/05/2013, per una densità di 5,3 territori $/ \mathrm{km}(\mathrm{FC})$;

- 6 individui in canto tra l'Alpe La Balma e Scaredi il 12/06/2013 (FC, LL, CM, PD);

- 10 individui in canto tra Pian d'Arla e Pian Vadà il 25/06/2013, per una densità di 3,7 territori/km (FC).

Nidificazioni accertate:

- 1 individuo che trasporta imbeccate presso il Bivacco Al Cedo il 15/6/1996 (CM);

- 1 individuo con imbeccata presso Cima Tuss il 16/6/2000 (CM);

- nido con pulcini in un bosco rado di larici con sottobosco di felci sul Monte Todum il 30/06/2002 (FC);

- 2-3 coppie (trasporto imbeccata) presso Biogna il 25/06/2013 (FC);

- coppia che trasporta imbeccate presso Bivacco Pian Vadà il 25/06/2013 (FC);

- 1 individuo con imbeccata a monte dell'Alpe Spoccia, in Val Cannobina, il 14/08/2014 (FC, MB).

In migrazione:

- il 28/08/2013, diretti a S: 2 individui in loc. Cresta della Ciresa, 1 individuo sul Monte Morissolino, 1 individuo presso il Passo Folungo (FC, $\mathrm{AG}$ );

- Bocchetta di Vald: 1 individuo il 13/09/2013 diretto a S (FC, CM, MP);

- Monte Carza, diretti a N: 1 individuo il 17/04/14 (FC, CM), 1 il 5/05/2014 (FC, MP);

- Scaredi: 5 individui diretti verso S 1'8/09/2014 (FC, MP);

- Colma di Premosello: 8 individui il 16/09/2014 (FC).

Si stima la presenza di 70-100 territori nell'area di studio (Fig. 22).

Pispola (Anthus pratensis) M reg

Specie che frequenta l'area di studio durante le migrazioni. Alcuni dati:

- Scaredi: 44 individui verso S il 18/10/2013 e 17 il 17/10/2014 (FC, MP);

- Colma di Premosello: 23 individui verso S il

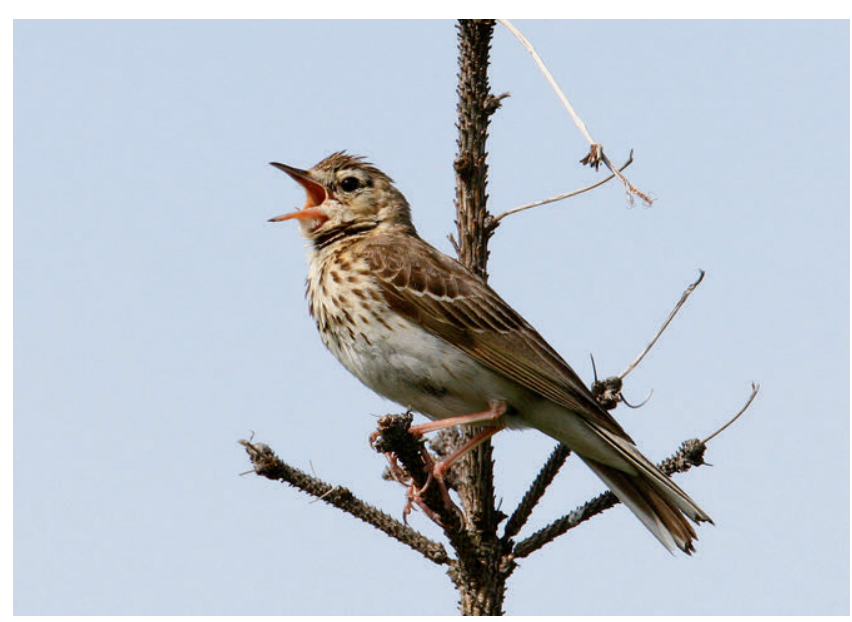

Fig. 22 - Prispolone. / Tree Pipit. (Foto / Photo Antonello Turri). 
25/10/2013 (FC, CM, MP, MD) e 4 individui il 3/10/2014 (FC, CM);

- Bocchetta di Vald: 1 individuo verso S il 30/10/2013 (FC, DR);

- Bocchetta di Cavalla: 5 individui verso $S$ il 30/10/2013 (FC, DR);

- Monte Carza: 2 individui verso N il 17/04/14 (FC, $\mathrm{CM})$.

\section{Spioncello (Anthus spinoletta) M reg, B}

Specie comune come nidificante negli ambienti adatti, meno diffusa del prispolone. 26 territori individuati nel 1993 su 115 punti d'ascolto, per una dominanza relativa dell'1,7\% (Movalli \& Grimaldi 1996). Casale e Brambilla (2010b) hanno rilevato densità elevate tra Cappella di Terza e l'Alpe Straolgio, con 6 territori in un'area di 23,6 ha, per una densità di $2,5 / 10$ ha.

In periodo riproduttivo è presente:

- tra Monte Zeda e Pizzo della Marona (almeno 2 individui con imbeccata il 29/06/2012 e 8 territori il 25/06/2013: FC);

- presso il Bivacco Pian Vadà (1 individuo con imbeccata il 29/06/2012: FC);

- tra Rifugio Parpinasca e Colma di Basagrana (3 coppie con giovani l'11/07/2014): FC, DR e FCA;

- alle pendici della Punta Tignolino (1 individuo con imbeccata l'11/07/2014: FC, DR e FCA);

- lungo la dorsale Monte Todano-Il Pizzo (4 individui in canto $1^{\prime} 8 / 05 / 2014$, pari a una densità di 4 territori / km: FC);

- presso Cappella di Terza (2 coppie allarmate il 30/06/2014: FC);

- lungo la dorsale Cimone di Straolgio-Pizzo Stagno (16 territori il 30/06/2014, pari a una densità di 3 territori $/ \mathrm{km}$ : FC).

In periodo post-riproduttivo: circa 75 individui nella zona di Scaredi-Cima della Laurasca-Lago del Marmo 1'8/09/2014 (FC, MP) e circa 50 individui tra Bivacco Pian Vadà e Monte Zeda il 10/10/2010 (FC).

In migrazione: 7 individui in transito a Scaredi il 18/10/2013, 5 alla Colma di Premosello il 25/10/2013 e 1 individuo alla Bocchetta di Vald il 30/10/2013 (Casale 2014).

\section{Cutrettola (Motacilla flava) M irr}

Una sola osservazione nota: 1 individuo in migrazione verso $\mathrm{S}$ a Scaredi l'8/09/14 (FC, MP).

\section{Ballerina gialla (Motacilla cinerea) SB}

La specie è piuttosto diffusa nell'area di studio e nidifica nei pressi dei corpi idrici. Cinque territori erano stati individuati nel 1993 su 115 punti d'ascolto, per una dominanza relativa dello $0,3 \%$ (Movalli \& Grimaldi 1996). Alcuni dati in periodo riproduttivo:

- Cossogno: 1 individuo con imbeccata il 24/05/2002 (FC);

- Alpe La Motta: 1 individuo sul rio sottostante il 24/06/2001 (CM), 1 individuo in canto l'11/06/2013 e il 3/07/2013 (FC, LL, CM, PD) e 1 individuo il 4/07/2014 (LL, MP);

- Rio Boschelli: 1 individuo in canto presso l'Alpe omonima il 12/06/2013 (FC, LL, CM, PD);
- RioPogallo: 1 individuoil24/06/2000(CMeMercedes Filippi), 1 individuo che trasporta l'imbeccata alla foce del Rio Pogallino il 17/06/2013 (FC, CM, ST);

- Val Caurì: una coppia il 25/07/2013 (BDVG);

- Pian di Boit: 3 individui il 17/06/2014 (LL, MP);

- Rio Pianezzoli: una coppia il 5/07/2014 (LL, MP);

- Cavaglio, in Val Cannobina: 1 individuo il 14/08/2014 (FC).

Si stima la presenza di 20-30 coppie.

Ballerina bianca (Motacilla alba) SB, M reg

La ballerina bianca nidifica soprattutto in prossimità dei centri abitati (ad es. 3-4 territori sono stati rilevati tra Finero e Provola 1'1/07/2009, confermando analoghi rilevamenti effettuati nel giugno del 2000: Casale \& Brambilla, 2010b; CM), mentre è scarsa negli ambienti naturali (una coppia nei pressi del Rifugio Pian Cavallone il 29/06/2012: FC; una coppia a Passo Folungo il 21/07/2009: Casale \& Brambilla, 2010b). Tale specie non è stata rilevata nei punti d'ascolto eseguiti nel 1993 da Movalli e Grimaldi (1996) e nemmeno nel 2013 (Casale, 2014) e nel 2014 (LL, MP) nell'ambito del progetto di "Monitoraggio della biodiversità".

Si stima la presenza di 10-20 coppie nidificanti nell'area di studio.

L'area è interessata anche da un flusso migratorio che interessa i passi montani:

- 3 individui diretti a S a Scaredi il 18/10/2013 (Casale, 2014);

- 1 individuo diretto a S alla Colma di Premosello il 25/10/2013 (Casale, 2014).

\section{Cinclidae}

\section{Merlo acquaiolo (Cinclus cinclus) SB}

Il merlo acquaiolo è presente lungo numerosi corsi d'acqua compresi nell'area di studio, a basse densità. Risulta più frequente lungo il Rio Cannobino. Quattro territori erano stati individuati nel 1993 su 115 punti d'ascolto, per una dominanza relativa dello $0,3 \%$ (Movalli \& Grimaldi, 1996).

Alcuni dati di presenza in periodo riproduttivo:

- Rio Boschelli: 1 individuo il 25/05/1997 (CM) e 1 individuo il 12/06/2013 (Casale, 2014);

- confluenza del Rio Val Rossa con il Rio Fiorina: 1 individuo che esibiva comportamento territoriale il 17/05/2000 (CM);

- Torrente San Giovanni, in Valle Intrasca: 1 individuo il 18/05/2000 tra Scareno e Intragna (FC);

- Rio Crot: 1 individuo sotto Alpe La Piana l'11/06/2010 e, sul medesimo rio, uno tra Alpetto Cornala e Alpe Basciot, il 17/04/2014 e 1 giovane da poco involato sotto Alpetto Cornala il 18/06/2014 (BDVG);

- Rio Val di Nass: 1 individuo il 31/03/2013(BDVG);

- Rio Valgrande: 1 individuo in Val Caurì il 25/07/2013 (BDVG);

- Rio Cannobino: una coppia presso il bivio per Gurro e 1 individuo presso il ponte più a monte il 5/06/2014 (FC);

- Rio Pianezzoli: 1 individuo il 17/06/2014 (LL, MP); 
- Rio Pogallo: 1 individuo tra Cicogna e Pogallo il 5/07/2014 (LL, MP).

Al di fuori del periodo riproduttivo:

- 1 individuo tra Scaredi e In La Piana il 26/10/1996 (FC);

- 2 individui in Val Rossa il 18/08/2010 (BDVG);

- 1 individuo al Ponte Casletto il 10/10/2011 (BDVG);

- 1 individuo presso l'Alpe Bondolo il 13/09/13 (FC, CM, MP) e il 30/10/2013 (FC, DR);

- 2 individui a Fondo Li Gabbi il 30/10/2013 (FC);

- 1 individuo in Val Gabbio il 22/09/2014 (BDVG).

Si stima la presenza di 10-15 coppie.

\section{Troglodytidae}

Scricciolo (Troglodytes troglodytes) SB, M reg, W reg

Specie molto comune nell'area di studio, dove occupa vari ambienti fino a $2000 \mathrm{~m}$ s.l.m., con predilezione per i versanti freschi e umidi. Ottantanove territori erano stati individuati nel 1993 su 115 punti d'ascolto, per una dominanza relativa del 5,8\% (Movalli \& Grimaldi, 1996). In periodo riproduttivo:

- coppia allarmata lungo il Rio Pogallo il 17/06/2013 (FC, CM, ST);

- adulto molto allarmato presso Cicogna l'11/07/2013 (FC, CM);

- coppia con giovani in loc. Fornaci, in alta Val Loana il 30/06/2014 (FC).

Elevate densità: 6 individui in canto tra Colma di Premosello e Alpe Serena il 3/07/2013, pari a 7,5 territori/ $\mathrm{km}(\mathrm{FC}, \mathrm{CM}) ; 19$ individui in canto tra Rifugio Parpinasca e Colma di Basagrana 1'11/07/2014, pari a 6,3 territori/km (FC, DR, FCA).

Si stima la presenza di $300-400$ territori nell'area di studio.

\section{Prunellidae}

Passera scopaiola (Prunella modularis) M reg, B, W reg Specie comune nell'area di studio, dove raggiunge le densità più elevate nella fascia arbustiva del rodorovaccinieto. Cinquanta territori erano stati individuati nel 1993 su 115 punti d'ascolto, per una dominanza relativa del 3,3\% (Movalli \& Grimaldi, 1996). Casale e Brambilla (2010b) hanno rilevato densità elevate a Scaredi, con 8 territori in un'area di 26,0 ha, per una densità di 3,1/10 ha.

Si stima la presenza di $100-200$ territori nell'area di studio.

\section{Sordone (Prunella collaris) SB}

Il sordone frequenta a basse densità gli ambienti rocciosi alle quote più elevate comprese nell'area di studio. Sei coppie erano state individuate nel 1993 su 115 punti d'ascolto, per una dominanza relativa dello $0,4 \%$ (Movalli \& Grimaldi, 1996).

In periodo riproduttivo sono note le seguenti segnalazioni:

- 1 individuo sopra i laghetti del Geccio il 15/06/1996 (CM);

- 1 individuo nei pressi della cima del Proman il 24/06/2001 (CM);
- 1 individuo al Pizzo della Marona il 09/07/2001 (CM e Mercedes Filippi);

- coppia con 4 giovani presso la Colma di Basagrana il 28/08/2012 (FC e AG);

- 5 individui sul Monte Zeda (due coppie, di cui una con maschio in canto, e un maschio singolo in canto) il 21/06/2012 (FC);

- 2 individui alla Colma di Premosello il 3/07/2013 (FC, CM);

- 1 individuo in canto alla Bocchetta di Terza il 17/06/2014 (LL, MP);

- 1 individuo alla Scala di Ragozzale il 18/06/2014 (MP, MD);

- 1 individuo in canto sul Pizzo Stagno ed 1 in canto in località Strecciùn (Trontano) il 30/06/2014 (FC);

- 2 individui l’11/07/2014 tra il Rifugio Parpinasca e la Colma di Basagrana (FC);

- 1 individuo sul Monte Togano 1'11/07/2014 (FC).

Al di fuori del periodo riproduttivo: 1 individuo sulla vetta della Cima della Laurasca il 6/10/2007 e 25/10/2012 (FC), 3 individui l'8/12/2003 e circa 20 individui il 16/12/2003 a metà percorso tra Cappella Fina e il Rifugio Pian Cavallone (FC), 2 individui ai Ronchi di Colloro 1' 1/01/2010 (BDVG), 1 individuo il 31/10/2010 presso la Colma di Premosello (FC), 1 individuo presso l'Alpe Ragozzale il 17/10/2011 (CM), 3 individui sotto Colloro il 7/02/12 (BDVG), 1 individuo a Biogno-Capraga il 26/02/2013 (BDVG), 1 individuo il 6/04/2013 nei pressi del Monte della Cima di Morissolo (RB, FC, CM, LP), stormo di 34 individui presso la Colma di Premosello il 25/10/2013 (FC, CM, MP e MD), 1 individuo sul Monte Carza (Trarego-Viggiona) il 27/03/2014 (FC), 1 individuo a Scaredi 1'8/09/2014 e il 17/10/2014 (FC, MP).

Si stima la presenza di 20-30 famiglie estese riproduttive nell'area di studio (Fig. 23).

\section{Turdidae}

Pettirosso (Erithacus rubecula) M reg, B, W

Una delle specie più comuni nell'area di studio, dove frequenta soprattutto le aree boscate e raggiunge la massima abbondanza intorno ai $1.100 \mathrm{~m}$ s.l.m. 132 coppie erano state individuate nel Parco nel 1993 su 115 punti

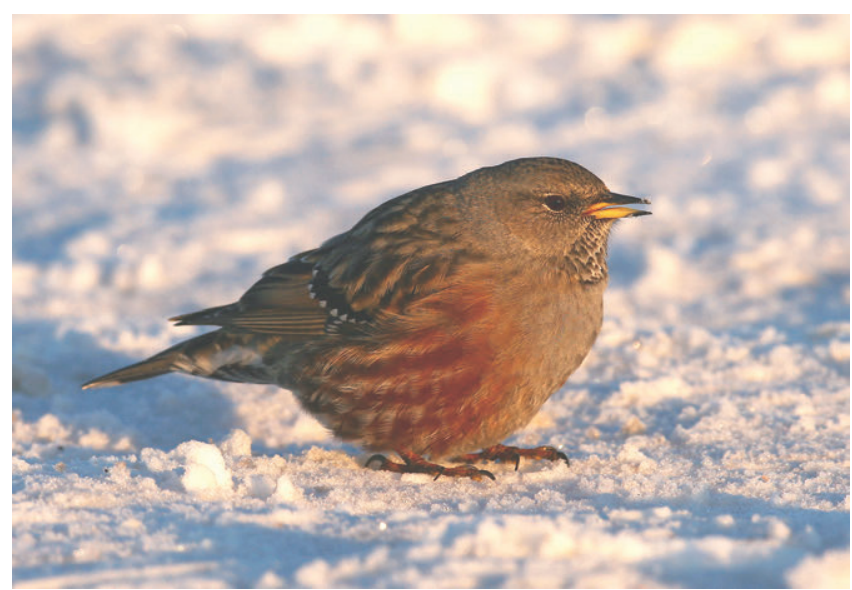

Fig. 23 - Sordone. / Alpine Accentor. (Foto / Photo Antonello Turri). 
d'ascolto, per una dominanza relativa dell' $8,6 \%$ (Movalli $\&$ Grimaldi, 1996). Durante i transetti realizzati nel mese di giugno per il progetto di "Monitoraggio della biodiversità" sono stati rilevati 29 maschi in canto nel 2013, mentre nel 2014 tale numero è sceso a 16 , verosimilmente a causa del forte innevamento che ha caratterizzato l'inverno 2013-2014 determinando un ritardo nella disponibilità di ambienti idonei alla nidificazione rispetto ad annate con innevamento nella norma. Durante i successivi rilevamenti del mese di luglio tali valori sono risultati confrontabili tra loro: 17 territori nel 2013 e 16 nel 2014.

Alcuni dati di nidificazione:

- nido con 5 uova nella cavità di un muretto a secco a circa 1,6 m. di altezza tra Rovegro e Cossogno il 17/07/2001 (FC);

- 3 giovani imbeccati dai genitori immediatamente a valle di Cicogna il 18/05/2013 (FC);

- 1 adulto allarmato presso l'Alpe Portaiola ed uno tra Alpe Portaiola e Alpe Boschelli il 12/06/2013 (FC, LL, CM, PD);

- 1 adulto allarmato in Val Pogallo, lungo il Rio Pianezzoli, il 17/06/2013 (FC, CM, ST);

- coppia con 3 giovani tra Alpe Marano e Cappella Lego (Cavaglio-Spoccia), in Val Cannobina, il 14/08/2014 (FC, MB).

Massime densità rilevate:

- 15 individui in canto tra Monte Todum e Cappella Fina il 18/05/2013, per una densità di 9,8 territori/ $\mathrm{km}$ (FC);

- 16 individui in canto nella pecceta tra Cappella Porta e Rifugio Pian Cavallone 1'8/05/2014, per una densità di 8,4 territori/km $(\mathrm{FC})$.

Si stima la presenza di 300-500 territori nell'area di studio.

In migrazione: 1 individuo a circa $2.000 \mathrm{~m}$ in ambiente arbustivo in località Pian Vadà il 10/10/2010 (FC).

Pettazzurro (Luscinia svecica) A-1 (2012)

Un maschio del primo anno in canto è stato rilevato il $21 / 06 / 2012$ in un'area sita a $1.840 \mathrm{~m}$ di altitudine la cui localizzazione non viene specificata per motivi conservazionistici (FC). Il canto era composto quasi esclusivamente da imitazioni. Sono stati riconosciuti i seguenti canti imitati: Codirosso spazzacamino (Phoenicurus ochruros), Fringuello (Fringilla coelebs), Cinciarella (Cyanistes caeruleus), Usignolo (Luscinia megarhynchos), ballerina bianca, Cinciallegra (Parus major), cavalletta (Orthoptera). L'habitat frequentato possedeva la seguente copertura: rododendro $(90 \%)$, rocce $(5 \%)$, prato $(5 \%)$, arbusti di ginepro e brugo $(<1 \%)$ e il sito era esposto a sud-est. Si tratta di un ambiente molto simile a quello in cui la specie ha nidificato nelle Alpi lombarde (Brembilla, 2012). Nello stesso habitat sono stati osservati passera scopaiola, spioncello, fagiano di monte e nei pressi Bigiarella (Sylvia curruca) e sordone. Durante un successivo sopralluogo avvenuto dopo 8 giorni, tale presenza non è più stata riscontrata.

Codirosso spazzacamino (Phoenicurus ochruros) M reg, W reg, B

Specie comune nell'area di studio, dove nidifica in corrispondenza di alpeggi, pietraie e altri ambienti rocciosi, oltre che in numerosi centri abitati. Trentuno territori erano stati individuati nel 1993 su 115 punti d'ascolto, per una dominanza relativa del 2,0\% (Movalli \& Grimaldi, 1996). Casale e Brambilla (2010b) hanno rilevato densità elevate a La Balma (4 territori in un'area campione di 16,3 ha, per una densità di 2,4/10 ha) e tra Fondo Li Gabbi e Cascine, in alta Val Loana (5 territori in un'area campione di 29,5 ha, per una densità di 1,7/10 ha).

Nidificazioni accertate:

- un nido occupato all'Alpe Erta il 15/6/1996 (CM);

- coppia con un giovane presso il Bivacco Pian Vadà il 21/06/2012 (FC);

- femmina con imbeccata presso l'Alpe La Motta (Premosello C.) l'11/06/2013 (FC);

- coppia con 3 giovani tra Costa dei Bagnoli e Colma di Basagarana l'11/07/2014 (FC, DR, FCA).

Si stima la presenza di 100-200 coppie nidificanti nell'area di studio.

Codirosso comune (Phoenicurus phoenicurus) M reg, B

Il codirosso comune nidifica prevalentemente nei centri abitati, anche di piccole dimensioni (Cicogna), compresi nell'area di studio, e occasionalmente in alpeggi (Pogallo) o su edifici isolati (Alpe Archìa). Otto territori erano stati individuati nel 1993 (nido occupato presso l'Alpe Scellina) su 115 punti d'ascolto, per una dominanza relativa dello 0,5\% (Movalli \& Grimaldi, 1996).

Si segnalano i seguenti dati di presenza in periodo riproduttivo:

- nido con pulcini a Intragna il 18/05/2000 (FC) e all'Alpe Archìa 1'11/06/2002 (FC);

- femmina che imbecca i giovani il 31/05/2013 (FC) e nido con pulcini su edificio l'11/07/2013 a Cicogna (FC, CM);

- femmina che trasporta sacca fecale il 21/05/2000 a Cossogno (CM e Fabio Copiatti);

- maschio con imbeccata a Cossogno il 20/06/2001 (FC), a Trontano 1'11/07/2014 (FC), all'Alpe Manegra il 17/05/2014 (FC, MP), a Crealla (Falmenta) il 5/06/2014 (FC);

- 3 individui in canto in $500 \mathrm{~m}$, tra Alpe Ompio e Rifugio Fantoli 1'8/05/2013 (FC);

- singoli maschi in canto a I Curt sopra Colloro il 26/6/1996 (CM), a Colloro 1'11/06/2013 (FC), presso l'Alpe La Piana (Premosello C.) l'11/06/2013 (FC, LL, CM, PD), a Pogallo il 17/06/2013 (FC, CM), all'Alpe Pala (Miazzina) 1'8/05/2014 (FC), alle pendici del Monte Carza il 17/05/2014 e il 24/05/2014 (FC, MP), in baite a est dell'Alpe Colle (Trarego-Viggiona) il 17/05/2014 (FC, MP).

Si stima la presenza nell'area di studio di 30-40 coppie nidificanti.

Stiaccino (Saxicola rubetra) M reg, B irr

Nel 1993, Movalli \& Grimaldi (1996), in un'indagine condotta tramite punti d'ascolto, riferiscono la presenza di 4 territori di tale specie nell'area protetta (1 territorio tra Alpe Basagrana e Passo dei Tre Uomini e 2 territori all'Alpe Basagrana il 26/06, 1 territorio sotto l'Alpe Vald il 27/06). Dai dati contenuti in Bionda \& Bordignon 
(2006) relativi al successivo periodo 1996-2005, la specie risultava nidificante nelle seguenti località:

- 1 maschio in canto a Pian Puzzo l'11/06/2002 (FC);

- coppia in allarme a Scaredi il 24/07/2004 (FC);

- alpeggi sopra Colloro (1 maschio in canto il 26/06/1996 a Corpic e uno il 21/06/1998 a valle di Corpic; CM);

- 1 maschio in canto al Bivacco Al Cedo il 15/06/1996 (CM).

Uno studio condotto nel 2009 sull'avifauna nidificante negli ambienti aperti del Parco non aveva portato a individuare siti di nidificazione della specie nelle aree ove era stata rilevata in precedenza e in altri siti potenzialmente idonei (Casale \& Brambilla, 2010b). Nel 2012, una coppia ha nidificato con successo (coppia con imbeccata a $1.726 \mathrm{~m}$ s.l.m.) lungo il versante meridionale del Monte Zeda (FC). La specie non è stata rilevata nel 2013 e 2014, se non durante la migrazione autunnale, a conferma del forte declino subito nell'area di studio (Fig. 24). Il trend di questa specie è, d'altro canto, negativo anche in altre aree limitrofe, quali l'Alpe Devero situata anch'essa nel Verbano Cusio Ossola, dove ha mostrato una contrazione della densità riproduttiva da 4,2 territori/10 ha nel 1997 a
2,2 territori/10 ha nel 2002 (Bionda \& Bordignon, 2006) e nel limitrofo Canton Ticino, dove la specie ha subito un crollo della popolazione nidificante nel 2008 e 2009 (Horch \& Spaar, 2010).

In migrazione:

- 5 individui in un felceto sul Monte Spalavera il 22/08/2013 (FC, AG);

- 1 individuo in località La Selva (Aurano) il 25/08/2014 (FC, LL);

- 2 individui presso l'Alpe Cortenuovo (Malesco) 1'8/09/2014 (FC, MP).

Saltimpalo (Saxicola torquata) M irr, B?

Una sola osservazione nota per l'area di studio: un maschio molto allarmato il 12/07/2012 in ambiente di brughiera a monte di Biogna, a $1.310 \mathrm{~m}$ s.l.m. (FC).

\section{Culbianco (Oenanthe oenanthe) M reg, B}

Nel Parco, Movalli \& Grimaldi (1996), a seguito di un'indagine condotta tramite punti d'ascolto nel 1993, riferiscono la presenza di 5 territori di tale specie nell'area protetta (3 dei quali tra La Balma e Scaredi il 15/06), per una dominanza relativa dello $0,3 \%$. Dai successivi

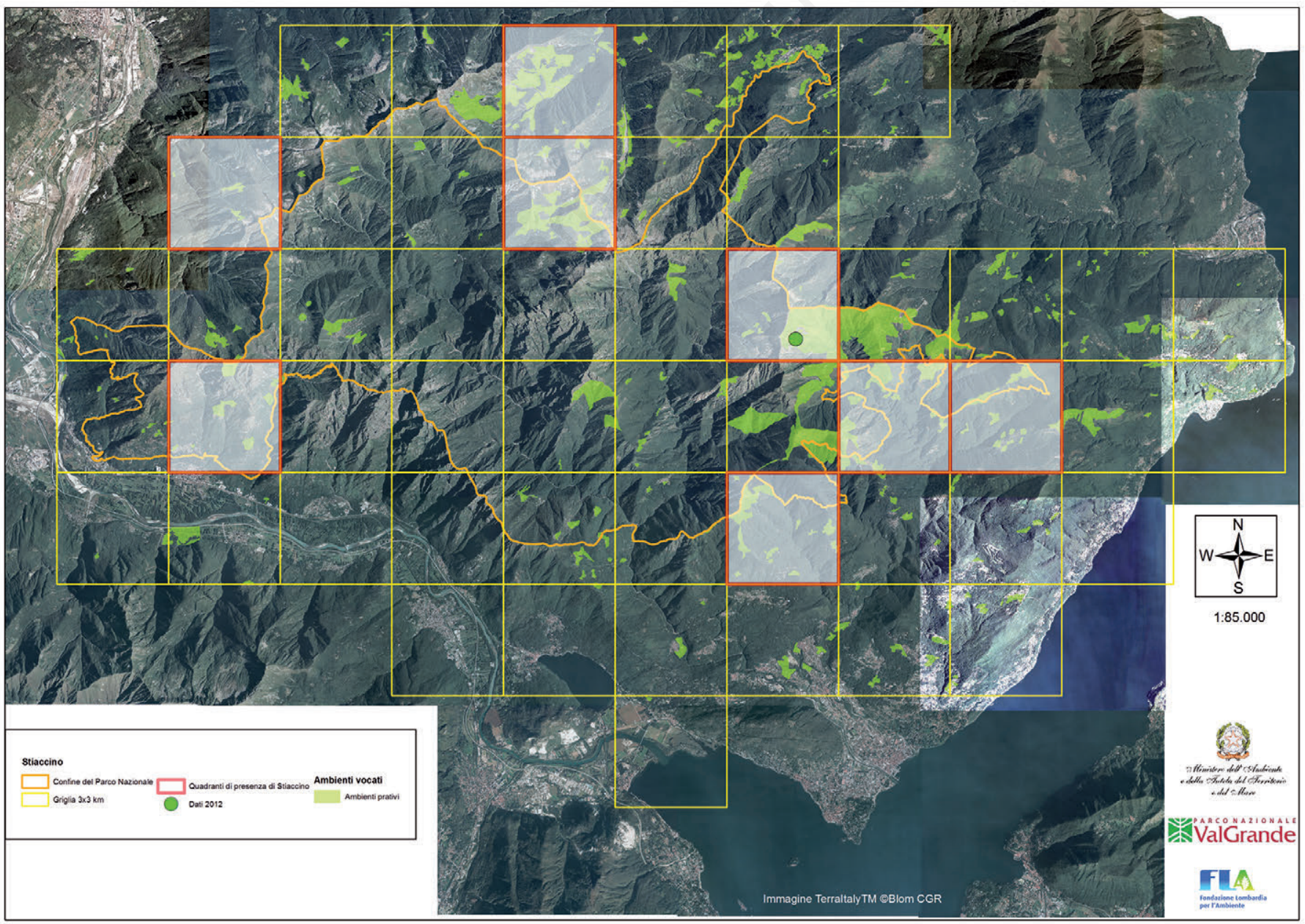

Fig. 24 - Stiaccino nel Parco Nazionale della Val Grande e aree limitrofe. Quadranti colorati: aree in cui la specie è stata rilevata in periodo riproduttivo nel 1993-2014; pallini verdi: dati di presenza in periodo riproduttivo nel 2012-2014. / Whinchat in Val Grande National Park and surrounding areas. Colored squares indicate were the species was recorded during the breeding season in 1993-2014 and green dots in 2012-2014. 
dati (1996-2005) dell'Atlante degli uccelli nidificanti del VCO la specie (Fig. 25) risultava nidificante in 5 tavolette comprese nel Parco della Val Grande (Bionda \& Bordignon, 2006) comprendenti una delle 4 Aree campione indagate nel 2009 nel corso dello studio sull'avifauna nidificante degli ambienti aperti (Casale \& Brambilla, 2009a), ovvero l'area di Scaredi-Straolgio-La Balma, ove invece il culbianco non è stato rilevato nel corso dell'indagine pur essendovi stato accertato come nidificante negli anni passati in località Scaredi (ad esempio, 1 maschio il 25/05/1997, una coppia in allarme il 17/06/2000, 1 maschio il 20/07/2001 (CM) e 2 maschi il 24/07/2004: FC e Giuliano Tallone). Nel 2011, viene osservata una coppia imbeccare due giovani nei pressi dell'Alpe Straolgio (22/07/2011: FC, LL, CM). Una coppia nidificante viene rilevata anche nel 2012, con genitori che portano imbeccate al nido nei pressi del Rifugio Pian Vadà (21/06/2012: FC).

Una specifica indagine condotta nel Parco in aree vocate per la specie nel corso delle stagioni riproduttive 2013-2014 ha portato a individuare 7 territori, per 5 dei quali è stato accertata la nidificazione:

- 1 territorio nei pressi del Rifugio Pian Vadà, dove già rilevato nel 2012 (accertata la nidificazione: adulti con imbeccata il 25/06/2013): FC;

- 1 territorio a Scaredi $(\mathrm{FC})$, dove già rilevato negli anni precedenti al 2009;

- 1 territorio presso Cappella di Terza (accertata la nidificazione: coppia con imbeccata il 4/07/2013: FC);

- 1 territorio sulla dorsale tra Cappella di Terza e Cimone di Straolgio (accertata la nidificazione: coppia con imbeccata il 4/07/2013 e il 30/06/2014: FC e CM);

- 1 territorio sulla dorsale sopra l'Alpe Straolgio (accertata la nidificazione: femmina con imbeccata l'1/07/2013: CM);

- 1 coppia allarmata presso Alpe Menta 1'11/07/2014 (FC, MD, DR, FCA);

- 1 coppia con 3 giovani presso l'Alpe Corte di Sopra il 21/08/2014 (MD).

Benché la tendenza nell'area indagata sembri essere

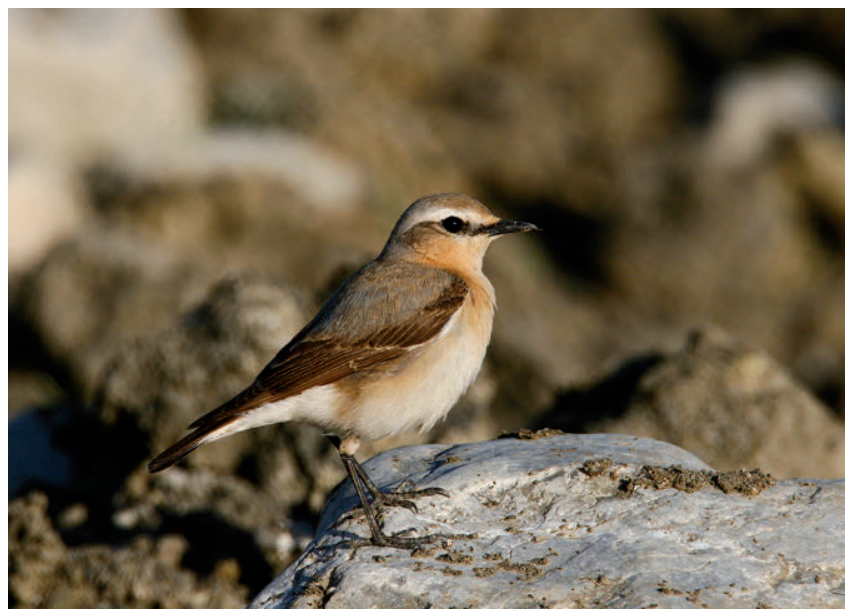

Fig. 25 - Culbianco. / Wheatear. (Foto / Photo Antonello Turri). negativa, in termini quantitativi, la distribuzione della specie non ha subito evidenti contrazioni negli ultimi 20 anni, come invece è avvenuto per lo stiaccino, verosimilmente in quanto quest'ultimo è specie maggiormente legata a prati-pascoli pingui, spesso nei pressi di alpeggi, soggetti a evidente ricolonizzazione erbacea e arbustiva negli ultimi anni, mentre il culbianco frequenta soprattutto praterie asciutte al di sopra del limite della vegetazione arboreoarbustiva (Bionda \& Bordignon, 2006; Fig. 26).

In migrazione:

- 2 individui presso il Passo Folungo il 10/10/2010 (FC);

- 1 individuo sul Monte Carza il 17/04/2014 (FC, CM), 2 il 5/05/2014 (FC, MP) e 1 individuo il 17/05/2014 (FC, MP);

- 1 individuo sul Monte Todano 1'8/05/2013 (FC);

- 5 individui tra Monte Todum e Rifugio Pian Cavallone il 18/05/2013 (FC);

- 1 individuo sulla Cima di Morissolo il 13/04/2014 (MP);

- stormo di 13 individui lungo la dorsale tra Monte Todano e Il Pizzo l'8/05/2014 (FC);

- 1 individuo all'Alpe Cortenuovo e 3 individui al Lago del Marmo, in alta Val Loana, l'8/09/2014 (FC, MP).

\section{Codirossone (Monticola saxatilis) MB}

A metà anni ' 90 del XX secolo, Movalli \& Grimaldi (1996) non citano la specie tra quelle rilevate nell'area protetta. Negli ultimi quindici anni, la specie è stata rilevata nell'area di Colloro il 13/6/1999 con un maschio in canto tra I Curt e Corpic (CM), sul Monte Todum (maschio in canto il 10/05/2002: FC) e nei pressi di Pian Puzzo (maschio in canto il 22/06/2006: FC), mentre lo studio condotto in aree campione nel 2009 non aveva permesso di confermare la presenza della specie (Casale \& Brambilla, 2010b). Nel 2012-2014 sono stati rilevati 4 territori entro i confini del Parco, e per 2 di essi è stata accertata la nidificazione:

- un territorio nei pressi di Pian Puzzo, dove già era stato rilevato nel 2006: 1 maschio con imbeccata il 21/06/2012, 1 maschio il 25/06/2013, una coppia di cui la femmina porta l'imbeccata il 15/06/2014 (FC);

- un territorio sulla dorsale sopra l'Alpe Straolgio, dove è stata osservata una coppia intenta a imbeccare 4 giovani il 3/07/2012 (LL);

- 2 maschi in canto lungo la dorsale Monte Todano Il Pizzo 1'8/05/2014 (FC).

Si stima la presenza di 5-10 territori nell'area di studio.

\section{Passero solitario (Monticola solitarius) SB}

Nell'area di studio, la specie è segnalata in periodo riproduttivo in alcune località poste sui versanti che si affacciano sul fondovalle ossolano, in corrispondenza di pareti rocciose sia naturali, sia sfruttate per l'estrazione lapidea in tempi più o meno recenti, nelle località Nibbio, Cuzzago, Beura-Cardezza, Croppo di Trontano (RB). All'interno dei confini del Parco sono note solo le seguenti segnalazioni:

- maschio in canto il 13/06/1999 presso l'Alpe I Curt $(\mathrm{CM})$; 
- coppia in località Sasso Bianco, in comune di Premosello-Chiovenda, negli anni '90 del XX secolo (E. Guenzi, com. pers.).

Si stima la presenza di 4-5 territori nell'area di studio.

Merlo dal collare (Turdus torquatus) M reg, B

Un territorio rilevato nel Parco nel 1993 da Movalli e Grimaldi (1996) su 115 punti d'ascolto. Negli anni successivi la sua presenza in periodo riproduttivo è stata riscontrata:

- nei pressi dell'Alpe La Balma (1 individuo il 25/05/1997: CM e Fabio Copiatti);

- tra Alpe Cortenuovo e Scaredi (1 individuo il 16/07/2009: Casale \& Brambilla, 2010b);

- tra Monte Zeda e Pizzo della Marona (1 individuo il 29/06/2012: FC);

- tra la Colma di Premosello e l'Alpe Serena (1 individuo l'8/06/2014: LL);

- tra Bocchetta di Vald e Alpe Vald di Sopra (1 individuo il 23/07/2014 (LL);

- 1 giovane il 12/09/2014 presso l'Alpe Rina (LL, MP).

Altre segnalazioni, in periodo post-riproduttivo:
- 1 individuo alla Bocchetta dell'Usciolo il 24/08/2012 (BDVG);

- 1 individuo alla Colma di Basagrana il 18/08/2013 (BDVG);

- 1 individuo alla Colma di Premosello il 25/09/2013 (BDVG) e 1 individuo nell'agosto 2014 (AM);

- 2 individui all'Alpe Campo di Sopra l'1/09/2014 (CM);

- 1 individuo presso l'Alpe Cortenuovo l'8/09/2014 (FC, MP);

- 1 individuo presso la Bocchetta di Terza il 13/09/2014 (AM).

Durante le migrazioni viene osservato anche a quote più basse:

- 2 individui all'Alpe Lut il 2/04/2013 (FC);

- 3 individui sulla Cresta della Ciresa il 6/04/2013 (RB, FC, CM);

- 2 individui sul Monte Carza il 12/04/2013 (RB, FC).

Si stima la presenza di 10-15 coppie nidificanti nell'area di studio.

Merlo (Turdus merula) SB, M reg, W reg

Si tratta di una delle specie più comuni nell'area di

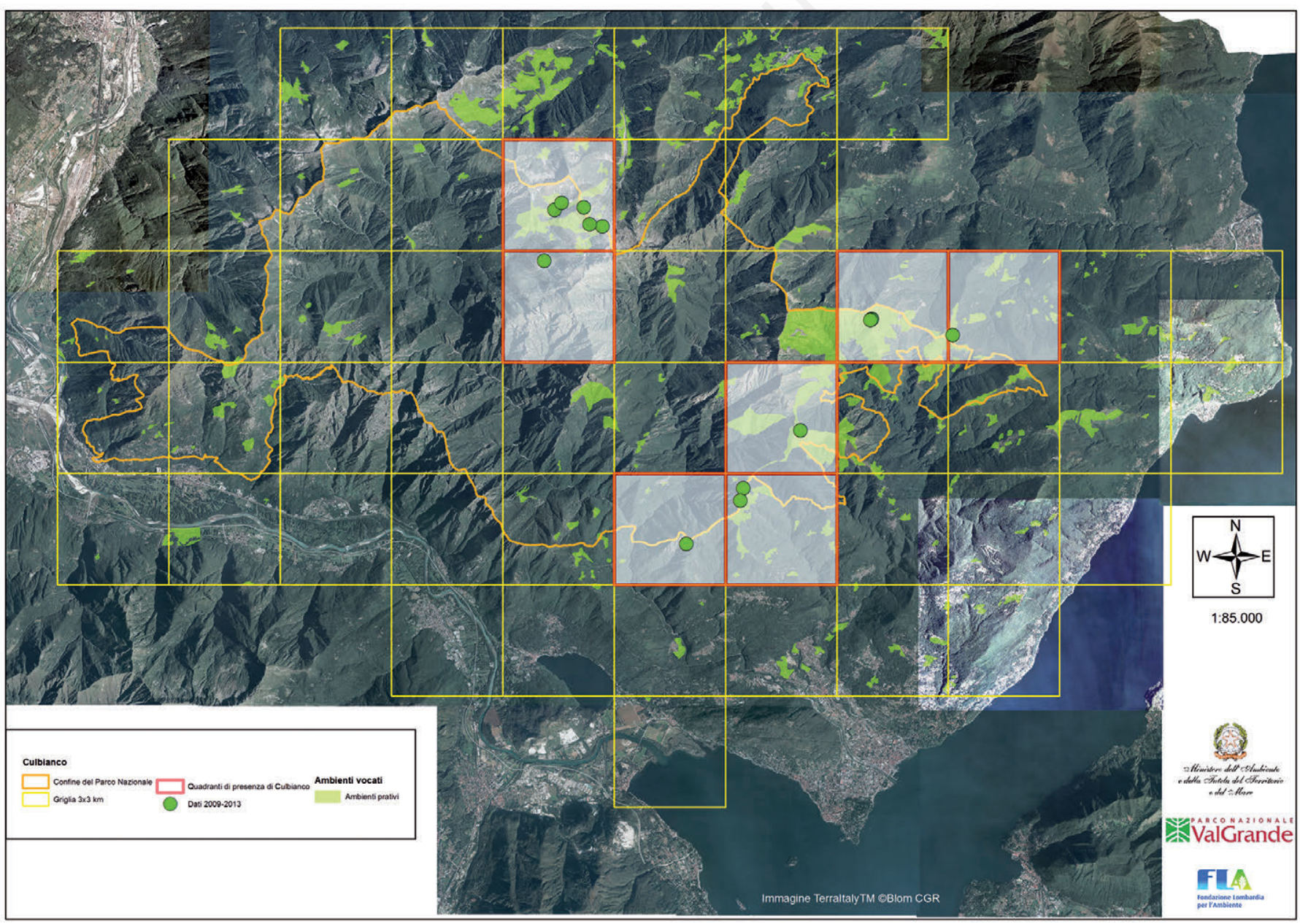

Fig. 26 - Culbianco nel Parco Nazionale della Val Grande e aree limitrofe. Quadranti colorati: aree in cui la specie è stata rilevata in periodo riproduttivo nel 1993-2013; pallini verdi: dati di presenza in periodo riproduttivo nel 2012-2013. / Wheatear in Val Grande National Park and surrounding areas. Colored squares indicate were the species was recorded during the breeding season in 1993-2013 and green dots in 2012-2013. 
studio. Settantuno territori individuati nel Parco nel 1993 su 115 punti d'ascolto, per una dominanza relativa del 4,6\% (Movalli \& Grimaldi, 1996). Analogamente a quanto precedentemente segnalato per il pettirosso, durante $\mathrm{i}$ transetti realizzati nel mese di giugno per il progetto di "Monitoraggio della biodiversità" sono stati rilevati 22 maschi in canto nel 2013, mentre nel 2014 tale numero è sceso a 19, verosimilmente a causa del forte innevamento che ha caratterizzato l'inverno 20132014, rendendo gli ambienti idonei alla nidificazione (in particolare gli habitat arboreo-arbustivi siti a quote più elevate) disponibili in ritardo rispetto ad annate con nevicate nella media.Durante i successivi rilevamenti del mese di luglio tali valori sono risultati confrontabili tra loro: 19 territori nel 2013 e 18 nel 2014.

Si stima la presenza di 200-300 coppie nidificanti nell'area di studio.

Cesena (Turdus pilaris) $\mathrm{M}$ reg, $\mathrm{W}$ reg

La cesena viene regolarmente rilevata nell'area di studio durante le migrazioni e in periodo invernale, mentre in periodo riproduttivo si è a conoscenza di un solo dato di presenza: 1 adulto trovato morto il 15/06/14 alla Bocchetta di Vald (LL).

In migrazione:

- uno stormo il 13/11/2007 a Straolgio (BDVG);

- stormo di 18 individui il 14/11/2008 tra la Colma di Premosello e il Pizzo Proman (BDVG);

- 3 individui il 10/10/2010 al Passo Folungo (FC);

- 4 individui a Capraga il 14/03/2011 (BDVG);

- 6 individui tra Fornaci e Scaredi il 4/10/2012 (BDVG);

- 1 individuo il 30/10/2013 presso la Bocchetta di Cavalla (FC, DR).

In inverno:

- 1 individuo all'Alpe Suì il 19/01/2011 (BDVG);

- 6 individui all'Alpe Sasso Termine 1'11/02/2011 (BDVG);

- 1 individuo all'Alpe Prà il 17/12/2013 (BDVG).

Tordo bottaccio (Turdus philomelos) M reg, B

Quattro territori individuati nel 1993 (Movalli \& Grimaldi 1996) su 115 punti d'ascolto. Diversamente da quanto precedentemente segnalato per merlo e pettirosso, durante i transetti realizzati nel mese di giugno per il progetto di "Monitoraggio della biodiversità" sono stati rilevati 12 maschi in canto sia nel 2013 che nel 2014. Una possibile spiegazione potrebbe consistere nel fatto che per tale specie la presenza di neve alle quote più elevate non ha inciso sugli ambienti idonei alla nidificazione, nel caso del tordo bottaccio rappresentati infatti quasi esclusivamente dalla faggeta localizzata a quote inferiori. Anche durante i successivi rilevamenti del mese di luglio, tali valori sono risultati confrontabili tra loro: 10 territori nel 2013 e 11 nel 2014 (Casale 2014; LL, MP). Massima densità rilevata: 5 individui in canto presso l'Alpe In La Piana 1'11/06/2013 (FC, LL, CM, PD) e il 3/07/2013 (FC, CM).

Si stima la presenza di 50-150 coppie nidificanti nell'area di studio.

\section{Tordo sassello (Turdus iliacus) M reg, W}

Poche le osservazioni note nell'area di studio: 1 individuo a Pian di Sole (Premeno) il 22/03/2002 (FC, E. \& L. Giussani), 1 individuo presso il Rifugio Fantoli il 6/03/2014 (FC, CM), 5 individui tra Fornaci e Alpe Cortenuovo in alta Val Loana il 31/10/2014 (CM).

Tordela (Turdus viscivorus) M reg, B

Specie non comune nell'area di studio come specie nidificante. Due territori individuati nel 1993 (Movalli \& Grimaldi 1996) su 115 punti d'ascolto. In periodo riproduttivo:

- 1 individuo il 22/6/1996 all'Alpe Caseracce (CM);

- 1 individuo il 21/06/2012 in loc. Cresta della Ciresa (FC);

- 1 individuo in canto presso Monte Todum (Cossogno) il 18/05/2013 (FC);

- 2 territori tra Alpe Portaiola e Alpe Boschelli il 12/06/2013 (FC, LL, CM, PD);

- 1 individuo in canto a Pian di Boit e una presso l'Alpe Terza, in Val Pogallo, il 17/06/2013 (FC, $\mathrm{CM}$;

- 1 individuo in canto presso Alpe La Piana (Premosello C.) il 6/07/2013 (FC);

- coppia allarmata in loc. Fornaci (Val Loana) il 30/06/2014 (FC);

- 3 individui in canto tra Alpe Ompio e Rifugio Fantoli il 6/03/2014 (FC, CM);

- 2 individui in canto sopra Cappella Porta (Caprezzo) il 20/03/2014 (FC, LL);

- 1 individuo in canto nella valle del Rio Cannero il 27/03/2014 (FC);

- 2 individui in canto sul Monte Carza il 24/05/2014 (FC, MP);

- 1 individuo che trasporta imbeccata al Pian dei Sali (Malesco) il 5/06/2014 (FC);

- 1 individuo presso l'Alpe Casarolo (Pizzo Mottac) il 18/06/2014 (MP, DR).

In migrazione:

- circa 15 individui in alta Val Loana il 30/10/1996 (FC);

- circa 50 individui tra Bivacco Pian Vadà e Monte Zeda il 10/10/2010 (FC);

- massimo di 62 individui in un unico gruppo tra Alpe Bondolo e Bocchetta di Vald il 30/10/2013 (FC, DR);

- 2 individui il 7/11/2014 a monte dell'Alpe La Motta (FC).

Si stima la presenza di 30-50 coppie nidificanti nell'area di studio.

\section{Sylviidae}

Canapino comune (Hippolais icterina) M irr, B irr

Pochissimi dati di presenza: 1 individuo in canto il 7/06/2002, presso il ponte di Santino (S. Bernardino V.), lungo il tratto terminale del torrente S. Bernardino (FC), in ambiente idoneo alla nidificazione; 1 individuo in canto il 25/05/2004 a Cossogno in un giardino, verosimilmente in migrazione (FC). 
Capinera (Sylvia atricapilla) SB, M reg

Specie molto comune nell'area di studio, raggiunge la massima abbondanza in corrispondenza delle aree di bosco con radure, soprattutto dove la vegetazione ruderale e arbustiva ha invaso gli alpeggi abbandonati. 127 territori sono stati individuati nel 1993 su 115 punti d'ascolto, per una dominanza relativa dell' $8,3 \%$ (Movalli \& Grimaldi 1996). Alcuni dati in periodo riproduttivo:

- giovane imbeccato dai genitori il 18/05/2013 a Miazzina (FC);

- elevata densità di 6 individui in canto 1'8/05/2014 in boscaglia di ricolonizzazione tra Cappella Fina e Rifugio Pian Cavallone (FC);

- maschio allarmato il 17/05/2014 presso l'Alpe Manegra (FC, MP).

Si stima la presenza di 300-500 coppie nidificanti nell'area di studio.

\section{Beccafico (Sylvia borin) M reg, B}

Specie comune negli ambienti adatti, ovvero arbusteti localizzati nella fascia di transizione tra vegetazione arborea e praterie alpine, in particolare in presenza di ontano verde. Cinquanta territori erano stati individuati nel 1993 su 115 punti d'ascolto, per una dominanza relativa del 3,3\%, e la massima abbondanza era stata riscontrata intorno ai $1.500 \mathrm{~m}$ s.l.m. (Movalli \& Grimaldi, 1996). Casale e Brambilla (2010b) hanno rilevato densità elevate in località Fornaci, in alta Val Loana (3 territori in un'area campione di 8,6 ha, per una densità di 3,5/10 ha) e tra Alpe Straolgio e La Balma (6 territori in un'area campione di 41,3 ha, per una densità di 1,4/10 ha).

In periodo riproduttivo:

- almeno 4 individui in canto tra Alpe Colle e Pian d'Arla il 12/07/2012 (FC);

- 1 individuo in canto presso Rifugio Pian Cavallone 1'8/05/2013 (FC);

- 1 individuo in canto presso l'Alpe Prà (Cossogno) il 31/05/2013 (FC);

- 1 individuo in canto presso l'Alpe Serena l'11/06/2013 (FC, LL, CM, PD);

- 1 individuo in canto presso l'Alpe Terza e uno presso la Bocchetta di Terza, in alta Val Pogallo, il 17/06/2013 (FC, CM, ST);

- 5 individui in canto tra Colma di Premosello e Alpe Serena il 3/07/2013 (FC, CM).

Si stima la presenza di $100-150$ coppie nidificanti nell'area di studio.

\section{Bigiarella (Sylvia curruca) M reg, B}

Otto territori individuati nel 1993 su 115 punti d'ascolto, per una dominanza relativa dello $0,5 \%$ (Movalli $\&$ Grimaldi, 1996). In periodo riproduttivo:

- 1 individuo in canto all'Alpe Quagiui il 10/6/2000 e il 6/07/2000 (CM);

- 1 individuo in canto in rodoreto tra Monte Zeda e Pizzo della Marona il 29/06/2012 (FC);

- 1 individuo in canto in rodoreto lungo la dorsale Monte Todano-Il Pizzo l'8/05/2013 (FC);

- 2 individui in canto in rodoreto alla Colma di Belmello il 31/05/2013 (FC);

- 4 territori presso La Balma il 3/07/2013 (FC, CM);
- 1 individuo in canto tra Pian Vadà e Monte Zeda il 15/06/2014 (FC);

- 1 individuo in canto a monte dell'Alpe Straolgio il 30/06/2014 (FC);

- 2 individui in canto presso la Colma di Basagrana e 1 individuo in canto presso il Passo dei Tre Uomini l'11/07/2014 (FC, DR, FCA).

Si stima la presenza di $30-40$ coppie nidificanti nell'area di studio.

Bigia grossa (Sylvia hortensis) A-1 (2004)

Una sola osservazione nota per l'area di studio: 1 maschio il 12/06/2004 in località Corte dei Galli, in Val Pogallo (FC e AG).

Sterpazzola (Sylvia communis) M reg, B?

Tre territori (2 nei pressi di Lut il 20/06 e 1 a valle di Caslù, in Val Pogallo, il 30/06) erano stati rilevati nel 1993 su 115 punti d'ascolto da Movalli \& Grimaldi (1996). L'unico dato successivo di presenza si riferisce a 2 individui presso l'Alpe Serena 1'8/06/2014 (LL, MP).

Sterpazzolina di Moltoni (Sylvia subalpina) A-1 (2014)

Un maschio in canto è stato osservato sul Monte Carza il 17/05/2014, in ambiente di ginestreto (FC). Successivi sopralluoghi non ne hanno riconfermato la presenza.

Luì bianco (Phylloscopus bonelli) M reg, B

Specie poco comune nell'area di studio come nidificante. Due territori individuati nel 1993 su 115 punti d'ascolto (Movalli \& Grimaldi, 1996). In periodo riproduttivo:

- 1 individuo in canto all'Alpe Ompio 1'11/06/1999 (CM);

- 1 individuo in canto nei pressi di Cima Tuss il 16/06/2000 (CM);

- 1 individuo in canto all'Alpe Busarasca il 31/5/2002 (CM);

- 1 individuo in canto il 24/07/1995 nella faggeta tra Fondo Li Gabbi e Alpe Loana, a circa 1000 m s.l.m. (FC, Giuliano Tallone);

- 1 individuo in canto nella Valle di Finero 1'1/07/2009 (Casale \& Brambilla, 2010b);

- 1 individuo in canto in pineta a Pino silvestre in loc. Pian dei Sali (Malesco) il 23/05/2009 e il 29/07/2012 (FC);

- 3 individui in canto sul Monte Carza 1'8/05/2013 (FC);

- 4 individui in canto in cerreto termofilo presso l'Alpe Prà (Cossogno) il 31/05/2013 (FC);

- 1 individuo in canto tra Lut e Alpe La Piana (Premosello C.) l'11/06/2013 e il 3/07/2013 (FC, LL, CM, PD);

- 1 individuo in canto presso l'Alpe Stavelli (Premosello C.) l'11/06/2013 (FC, LL, CM, PD);

- 1 individuo in canto presso l'Alpe La Motta (Premosello C.) il 3/07/2013 (FC, CM);

- 4 individui in canto in una boscaglia termofila tra Cappella Fina e Rifugio Pian Cavallone 1'8/05/2014 (FC).

In migrazione: 1 individuo in località Cresta della Ciresa (Premeno) il 28/08/2013 (FC, AG). 
Si stima la presenza di 30-50 coppie nidificanti nell'area di studio.

\section{Luì verde (Phylloscopus sibilatrix) $\mathrm{M}$ reg, $\mathrm{B}$}

Specie non comune nell'area di studio come nidificante. Un solo territorio individuato nel Parco su 115 punti d'ascolto, presso Cicogna il 17/05/1993 (Movalli \& Grimaldi, 1996). In periodo riproduttivo:

- 3 individui in canto tra Alpe Prà e Colma di Belmello il 7/05/1994 (FC e Ian Mifsud);

- 1 individuo in canto a Inoca, in comune di Cossogno l'1/06/1999 (CM);

- 1 individuo in canto all'Alpe Pala (Miazzina) il 18/05/2013 (FC);

- 1 individuo in canto nella Valle di Finero 1'1/07/2009 (Casale \& Brambilla, 2010b);

- 1 individuo in canto in Valle Intrasca, tra Sardella e Aurano, il 21/06/2012 (FC);

- 1 individuo in canto in loc. Basciotta (Cannero) il 5/06/2014 (FC, MP).

In migrazione: un maschio in canto tra Aurano e La Colla il 16/04/2000 (FC), 1 maschio al Pian dei Sali ed 1 maschio a Provola il 26/04/2011 (FC), 1 maschio all'Alpe Ompio l'8/05/2013 (FC).

Si stima la presenza di $10-20$ coppie nidificanti nell'area di studio.

Luì piccolo (Phylloscopus collybita) SB, M reg, W reg

Specie comune nell'area di studio, dove frequenta gli ambienti ecotonali e raggiunge la massima abbondanza dove il bosco, intorno ai $1.400 \mathrm{~m}$ s.l.m., tende a diradarsi. Novantadue territori sono stati individuati nel 1993 su 115 punti d'ascolto, per una dominanza relativa del 6,0\% (Movalli \& Grimaldi, 1996). In periodo riproduttivo, la massima densità è stata rilevata lungo il sentiero tra Cappella Fina e Rifugio Pian Cavallone in corrispondenza di pascoli abbandonati, con 8 maschi in canto il 18/05/2013 per una densità pari a 4 territori $/ \mathrm{km}(\mathrm{FC})$. Altre segnalazioni:

- 2 maschi in canto tra Lut e Alpe La Piana l'11/06/2013 (FC, LL, CM, PD);

- 1 maschio in canto presso l'Alpe Boschelli il 12/06/2013 (FC, LL, CM, PD);

- 2 maschi in canto in alta Val Loana il 12/06/2013 (FC, LL, CM, PD);

- 1 maschio in canto presso l'Alpe Terza, in Val Pogallo il 17/06/2013 (FC, CM).

In migrazione: almeno 7 individui nella Valle del Basso (Malesco) e 1 presso l'Alpe Vald di Sopra il 13/09/2013 (FC, CM, MP).

Si stima la presenza di 200-300 coppie nidificanti nell'area di studio.

\section{Luì grosso (Phylloscopus trochilus) $\mathrm{M}$ reg}

Sono note solo le seguenti osservazioni, verosimilmente in quanto durante la migrazione primaverile (quando la specie è facilmente contattabile al canto) gran parte dell'area di studio risulta inaccessibile:

- 2 individui in località Cresta della Ciresa (Premeno) il 28/08/2013 (FC, AG);

- 1 individuo sotto l'Alpe Cortenuovo (Malesco) 1'8/09/2014 (FC, MP).
Regolo (Regulus regulus) M reg, B, W reg

Otto territori individuati nel 1993 su 115 punti d'ascolto, per una dominanza relativa dello $0,5 \%$ (Movalli \& Grimaldi, 1996). Massima densità in periodo riproduttivo: 18 maschi in canto l' $8 / 05 / 2013$ nella pecceta tra Cappella Porta e Rifugio Pian Cavallone, per una densità pari a 9 territori/km (FC). Altri dati: 1 maschio in canto in un'abetina tra l'Alpe Portaiola e l'Alpe Boschelli il 12/06/2013 (FC, LL, CM, PD), coppia con giovani tra La Colletta e Rio Valgabbio, in bosco misto di faggio e abete bianco, il 3/07/2013 (FC, CM).

In migrazione: 1 individuo in transito alla Bocchetta di Vald il 30/10/2013 (Casale, 2014) ed uno il 7/11/2014 alla Colma di Premosello (FC, CM).

Si stima la presenza di 50-70 coppie nidificanti nell'area di studio.

\section{Fiorrancino (Regulus ignicapilla) M reg, B}

Specie apparentemente non comune nell'area di studio. Nel 1993, la specie veniva segnalata come presente nel Parco con 4 territori su 115 punti di ascolto da Movalli \& Grimaldi (1996), per una dominanza relativa dello 0,3\%. Alcuni dati in periodo riproduttivo:

- una coppia presso l'Alpe Ompio il 19/04/1997 (CM);

- 2 maschi in canto in pineta a Pino silvestre in loc. Pian dei Sali (Malesco) il 23/05/2009 (FC);

- 1 maschio in canto sul Monte Todum il 30/06/2002 (FC);

- 3 maschi in canto a Bieno l'8/05/2013 (FC);

- 1 maschio in canto nella pecceta sopra Cappella Porta (Caprezzo) l' $8 / 05 / 2013$ (FC);

- 4 maschi in canto nella pecceta tra Monte Todum e Cappella Fina il 18/05/2013 (FC);

- 1 maschio in canto a Ponte Spoccia e uno in canto in loc. La Piazza (Gurro) il 5/06/2014 (FC);

- 1 maschio in canto a Malesco il 30/06/2014 (FC).

In migrazione: 1 individuo tra Alpe La Piana e Alpe La Motta (Premosello C.) il 31/10/2010 (FC), 1 individuo tra Caprezzo e Alpe Pont il 20/03/2014 (FC, LL).

Si stima la presenza di 20-30 coppie nidificanti nell'area di studio.

\section{Muscicapidae}

Pigliamosche (Muscicapa striata) M reg, B

Specie non comune nell'area di studio, legata ai centri abitati e ai margini delle aree boscate. La specie nel 1993 veniva segnalata come presente nel Parco con 4 territori su 115 punti di ascolto da Movalli \& Grimaldi (1996), per una dominanza relativa dello $0,3 \%$. Alcuni dati in periodo riproduttivo:

- 1 giovane il 12/07/01 (FC) e un adulto con imbeccata il 30/06/2002 a Cossogno (FC);

- coppia con giovani a Biogna (Aurano) i1 21/07/2009 (Casale \& Brambilla, 2010b);

- 2 a Miazzina il 18/05/2013 (FC);

- 1 maschio in canto a Cicogna il 17/06/2013 (FC, $\mathrm{CM}, \mathrm{ST}$ );

- 1 maschio in canto a Manegra il 25/06/2013 (FC);

- nido su edificio con almeno 3 pulcini a Cicogna l'11/07/2013 (FC, CM); 
- 1 maschio in canto a Orasso il 5/06/2014 (FC);

- 1 individuo all'Alpe Marano, in comune di Cavaglio-Spoccia, il 14/08/2014 (FC).

Si stima la presenza di 10-20 coppie nidificanti nell'area di studio.

\section{Balia nera (Ficedula hypoleuca) M reg}

Specie comune nell'area di studio durante la migrazione autunnale. Alcuni dati:

- 2 individui il 15/09/2001 tra Cappella Fina e Rifugio Pian Cavallone (FC, Jean Marc Lance);

- 1 individuo il 22/08/2013 alle pendici del Monte Spalavera (FC, AG);

- 2 individui il 28/08/2013 in loc. Cresta della Ciresa (Premeno) (FC, AG);

- almeno 8 individui nella Valle del Basso il 13/09/2013 (FC, CM, MP);

- 1 individuo presso l'Alpe Marano (CavaglioSpoccia), in Val Cannobina, il 14/08/2014 (FC, MB);

- 3 individui in alta Val Loana, tra Fornaci e Alpe Cortenuovo, 1'8/09/2014 (FC, MP).

\section{Aegithalidae}

\section{Codibugnolo (Aegithalos caudatus) SB, M reg, W reg}

Specie comune ma apparentemente non abbondante come nidificante nell'area di studio, ove è stato rilevato in periodo riproduttivo fino a $1.400 \mathrm{~m}$ di altitudine. Quattordici territori erano stati individuati nel 1993 su 115 punti d'ascolto, per una dominanza relativa dello 0,9\% (Movalli \& Grimaldi, 1996). Alcuni dati in periodo riproduttivo: 1 individuo che trasporta materiale per il nido il 24/03/2002 tra Ungiasca e Cossogno (FC), coppia con imbeccata il 25/04/2002 all'Alpe Pala (Miazzina, FC).

Si stima la presenza di 70-100 coppie nidificanti nell'area di studio.

\section{Paridae}

Cinciarella (Cyanistes caeruleus) SB, M reg, W reg

Specie comune nell'area di studio, con 37 territori individuati nel 1993 su 115 punti d'ascolto per una dominanza relativa del 2,4\% (Movalli \& Grimaldi, 1996). Nidificazioni accertate: adulto con due giovani a Velina Baserga e 1 adulto con 1 giovane a Uccigiola il 16/06/2000 (CM), coppia con giovani da poco involati a Pogallo l'11/07/2013 (FC, CM).

In migrazione: 6 individui all'Alpe Cortenuovo, in alta Val Loana, in migrazione verso S l'8/09/2014 (FC, MP).

Si stima la presenza di 100-200 coppie nidificanti nell'area di studio.

\section{Cinciallegra (Parus major) SB, M reg, W reg}

Specie comune nell'area di studio, con 63 territori individuati nel 1993 su 115 punti di ascolto per una dominanza relativa del 4,0\% (Movalli \& Grimaldi, 1996). In periodo riproduttivo è stata rilevata fino a $1.400 \mathrm{~m}$ s.l.m. (maschio in canto presso l'Alpe Terza, in Val Pogallo, nel 2013; FC, CM). Alcuni dati in periodo riproduttivo: nido con pulcini a Cicogna il 7/05/1994 (FC, Ian Mifsud), nido occupato all'Alpe I Curt il 26/06/1996 (CM), nido con pulcini presso l'Istituto Auxologico di Piancavallo il 14/07/2001 (FC), coppia allarmata all'Alpe La Piana (Premosello C.) 1'11/06/2013 (FC, LL, CM, PD), nido con pulcini in località Cresta della Ciresa il 28/08/2013 (data piuttosto tardiva: $\mathrm{FC}, \mathrm{AG}$ ).

Si stima la presenza di 200-300 coppie nidificanti nell'area di studio.

\section{Cincia dal ciuffo (Lophophanes cristatus) SB}

Specie non comune e localizzata nell'area di studio, in corrispondenza dei nuclei di conifere di origine naturale (pino uncinato, abete bianco, larice) o artificiale (abete rosso), quali si riscontrano nelle località Cappella Fina (Miazzina) e Cappella Porta (Caprezzo). La specie era stata segnalata nel Parco nel 1993 con 4 territori su 115 punti di ascolto per una dominanza relativa del 4,1\% (Movalli \& Grimaldi, 1996). Alcuni dati in periodo riproduttivo: 1 individuo il 23/03/2002 sul Monterosso (Verbania) (FC), coppia con 4 giovani a Provola l'1/07/2009 (FC), 1 individuo tra La Colletta e Rio Val Gabbio in bosco misto di faggio e abete bianco il 3/07/2013 (FC, CM). Massima densità riscontrata: 4 territori nella pecceta tra Cappella Porta e Rifugio Pian Cavallone 1'8/05/2013, pari a 2,0 territori/km (FC).

Si stima la presenza di $30-50$ coppie nidificanti nell'area di studio.

Cincia mora (Periparus ater) SB, M reg, W reg

Specie comune nell'area di studio, segnalata come presente nel Parco nel 1993 con 60 territori su 115 punti di ascolto per una dominanza relativa del 3,9\% (Movalli \& Grimaldi, 1996). Frequenta sia boschi di conifere che boschi di latifoglie (faggeta). Alcuni dati in periodo riproduttivo:

- adulto con 2 giovani all'Alpe Ceresa il 17/06/2000 (CM e Fabio Copiatti);

- coppia che trasporta materiale per il nido ad Aurano il 18/05/2000 (FC);

- nido con pulcini a Inoca (Cossogno) il 21/05/2000 (CM e Fabio Copiatti);

- nido occupato a Cossogno il 05/05/2001 (FC);

- 1 adulto con imbeccata il 30/06/2002 sul Monte Todum (FC);

- 1 maschio in canto il 12/06/2013 e il 3/07/2013 presso l'Alpe In La Piana in ambiente di faggeta (FC, CM);

- 1 maschio in canto a Pian di Boit il 17/06/2013 (FC, CM, ST);

- una coppia tra La Colletta e Rio Valgabbio il 3/07/13 in bosco misto di faggio e abete bianco (FC, CM);

- 1 maschio in canto in faggeta pura 1'11/07/2013 a monte di Pian di Boit (FC, CM).

Massima densità: 6 territori nella pecceta tra Cappella Porta e Rifugio Pian Cavallone 1'8/05/2013, pari a 3,0 territori $/ \mathrm{km}$ (FC). Al di fuori del periodo riproduttivo, 1 individuo a $2.156 \mathrm{~m}$ s.l.m. sulla vetta del Monte Zeda il 10/10/2010 (FC).

Si stima la presenza di 200-300 coppie nidificanti nell'area di studio. 


\section{Cincia alpestre (Poecile montanus) SB}

Specie non comune e localizzata nell'area di studio, in corrispondenza dei nuclei di conifere di origine naturale (larice, pino uncinato, abete bianco) o artificiale (abete rosso) quali le località Cappella Fina (Miazzina; ad esempio, una coppia 1'11/05/2002: FC), tra Cappella Fina e Rifugio Pian Cavallone (una coppia il 20/03/2014: FC, LL; 2 coppie 1'8/05/2014: FC), Cappella Porta (Caprezzo), tra Rifugio Parpinasca e Colma di Basagrana (1 individuo 1'11/07/2014: FC), tra Colma di Premosello e Alpe Serena, tra Alpe Bondolo e Bocchetta di Cavalla (Malesco). In periodo post-riproduttivo osservata anche in ambiente di faggeta: 2 individui il 16/09/2014 e il successivo 7/11/2014 a 1.270 m s.l.m. tra Alpe La Motta e Alpe Stavelli (FC). La specie è stata rilevata da Movalli \& Grimaldi (1996) nel 1993 con un territorio su 115 punti di ascolto.

Si stima la presenza di $15-20$ coppie nidificanti nell'area di studio.

\section{Cincia bigia (Poecile palustris) SB}

Specie comune nell'area di studio fino a $1.400 \mathrm{~m}$ di altitudine, rilevata con 37 coppie su 115 punti di ascolto per una dominanza relativa del 2,4\% (Movalli \& Grimaldi, 1996). Alcuni dati in periodo riproduttivo: un nido nei pressi di Bieno (S. Bernardino) il 09/07/2001 (FC); 1 individuo con imbeccata il 23/03/2002 sul Monterosso (Verbania) (FC); famiglia con giovani in Val Pogallo, lungo il Rio Pianezzoli, il 17/06/2013 (FC, CM); coppia allarmata presso l'Alpe Preda di Qua, in Val Pogallo, il 17/06/2013 (FC, CM); coppia con giovani da poco involati presso l'Alpe La Motta (Premosello C.) il 6/07/2013 (FC).

Si stima la presenza di 200-300 coppie nidificanti nell'area di studio (Fig. 27).

\section{Sittidae}

\section{Picchio muratore (Sitta europaea) SB}

Specie comune nell'area di studio in aree boscate con presenza di vecchi alberi, rilevata con 8 territori su 115 punti di ascolto per una dominanza relativa dello $0,5 \%$

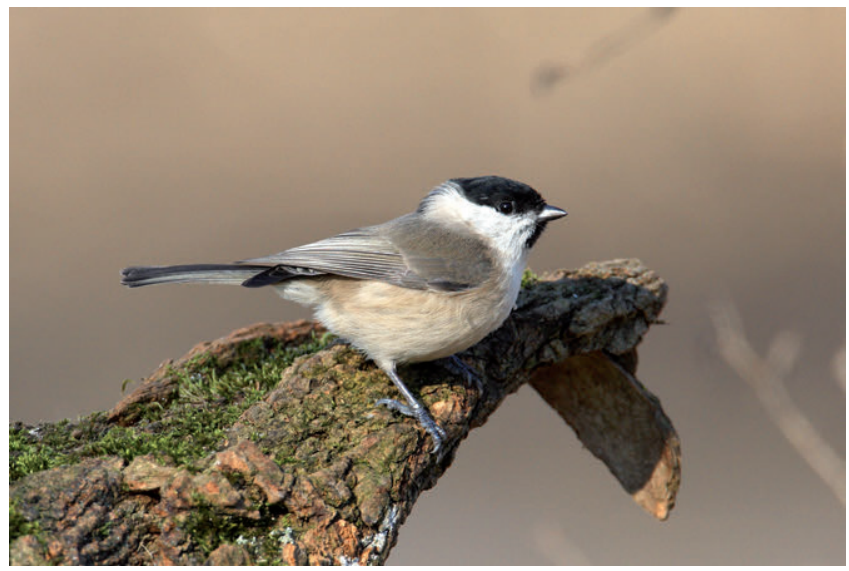

Fig. 27 - Cincia bigia. / Marsh Tit. (Foto / Photo Antonello Turri).
(Movalli \& Grimaldi, 1996). Il numero non elevato di territori censiti anche nei punti di ascolto realizzati nel 2013 e 2014 è ascrivibile al fatto che i rilievi sono stati effettuati tra giugno e luglio e non nei mesi precedenti, nei quali la specie è più facilmente contattabile, a causa dell'inaccessibilità di gran parte dell'area protetta per la presenza di neve.

Si stima la presenza di 100-200 coppie nidificanti nell'area di studio.

\section{Tichodromidae}

\section{Picchio muraiolo (Tichodroma muraria) SB}

Il picchio muraiolo nidifica nell'area di studio con un ridotto numero di coppie (5-10), ma la bassa densità di territori è caratteristica della specie (Bocca \& Maffei, 1997). Sono note le seguenti osservazioni:

- 1 individuo a Bocchetta di Terza il 25/06/2000 (CM e Mercedes Filippi);

- 1 individuo presso il Rifugio Bocchetta di Campo nel settembre 2001 (Fabio Sperotto, com. pers.);

- 1 individuo sul tetto del Palazzo pretorio di Vogogna il giorno 10/12/2009 (Massimo Scanzio, com. pers.);

- 2 individui all'Alpe Stavelli 1'1/10/2010 (BDVG);

- 1 individuo a Cappella di Terza il 18/10/2010 (BDVG);

- una coppia presso il Pizzo Dioisi il 15/06/2012 (BDVG);

- una coppia in atteggiamento riproduttivo nel giugno 2012 in loc. Strecciùn, presso il Pizzo Stagno (DR);

- 1 individuo alla Bocchetta di Vald il 30/10/2013 (FC, DR);

- 1 individuo alla Bocchetta di Terza il 17/06/2014 (LL, MP);

- 3 individui il 30/09/2014 (Maria Teresa Ciapparella, com. pers.) e 2 il successivo 18/10/2014, nei pressi di Passo Folungo (CM, MP).

\section{Certhiidae}

\section{Rampichino alpestre (Certhia familiaris) SB}

Specie non comune e localizzata nell'area di studio, soprattutto in corrispondenza dei nuclei di conifere di origine naturale (pino uncinato, abete bianco, larice) o artificiale (abete rosso), quali le località Cappella Porta (Caprezzo, 1 maschio in canto il 20/03/2014: FC, LL), Cappella Fina (Miazzina, 1 maschio in canto 1'11/05/2002: FC), pecceta tra Cappella Fina e Rifugio Pian Cavallone (1 maschio in canto il 18/05/2013: FC; 2 maschi in canto il 20/03/14: FC, LL; 2 maschi in canto il 18/05/2014: FC), Rifugio Fantoli (S. Bernardino V., 1 maschio in canto il 6/03/2014: FC, CM), Alpe Rina (Trontano, 1'11/07/2014 1 adulto che trasporta imbeccata; FC, MD, DR e FCA), ma anche in ambiente di faggeta pura, ad esempio presso l'Alpe In La Piana (1 maschio in canto il 12/06/2013 e il 3/07/2013: FC, CM); tra l'Alpe In La Piana e l'Alpe Portaiola (3 maschi in canto il 12/06/2013: FC, LL, CM, PD); presso l'Alpe Portaiola (coppia con imbeccata il 3/07/2013: FC, CM); tra Alpe Preda di Là e Pian di Boit, in 
Val Pogallo (1 maschio in canto il 17/06/2013: FC, CM).

Si stima la presenza di 20-30 coppie nidificanti nell'area di studio.

Rampichino comune (Certhia brachydactyla) SB

Specie comune nell'area di studio in aree boscate con presenza di vecchi alberi fino a $1.230 \mathrm{~m}$ s.l.m., rilevata nel 1993 con 10 territori su 115 punti di ascolto per una dominanza relativa dello $0,6 \%$ (Movalli \& Grimaldi, 1996). Analogamente al picchio muratore, il numero non elevato di territori censiti anche nei punti di ascolto realizzati nel 2013 e 2014 è ascrivibile al fatto che i rilievi siano stati effettuati tra giugno e luglio, e non nei mesi precedenti nei quali la specie è più facilmente contattabile, a causa dell'inaccessibilità di gran parte dell'area protetta per la presenza di neve.

Si stima la presenza di 100-200 coppie nidificanti nell'area di studio.

\section{Oriolidae}

\section{Rigogolo (Oriolus oriolus) M irr}

Poche segnalazioni: 1 maschio in canto in località Cappella Fina il 18/05/2013 (FC) e 1 maschio in canto il 24/05/2014 nella valle del Rio Cannero (FC, MP).

\section{Laniidae}

Averla piccola (Lanius collurio) $\mathrm{M}$ reg, B

I primi dati relativi alla presenza della specie nel Parco si riferiscono al 1993, con 6 territori (5 localizzati nelle alpi sopra Colloro e 1 presso Cicogna, in loc. Merina) complessivamente rilevati in 115 punti d'ascolto per una dominanza relativa dello $0,4 \%$ (Movalli e Grimaldi, 1996). Per gli anni successivi sono note le seguenti osservazioni: 1 maschio all'Alpe I Curt il 21/06/1998 e il 13/06/1999 (CM), 1 maschio a Finero il 20/06/2000 (CM). Un successivo studio realizzato nel 2009 (Casale e Brambilla, 2010b) e focalizzato su tutte le aree del Parco maggiormente vocate ad ospitare la specie ha portato alla individuazione di 4 territori; 3 di essi erano localizzati in un'area a pascolo arbustato e brughiera di 35,7 ha nei pressi di Biogna (Aurano), tra i 995 e i 1.286 m, s.l.m. per una densità di 0,8 coppie/10 ha. Il quarto territorio è stato invece localizzato nei pressi di Finero, in un'area caratterizzata dalla presenza di prati da fieno ben conservati con presenza di arbusti e alberi isolati. Successivamente è stato osservato un maschio il 29/05/2011 a Bracchio, in comune di Mergozzo (CM). Nel corso della stagione riproduttiva 2013 (Casale, 2014) sono state monitorate tutte le aree idonee ad ospitare la specie, già oggetto di indagine nel corso del 2009, ed alcune altre aree meno idonee, ed è stata rilevata la presenza di un solo territorio, in località Biogna, a conferma del trend negativo a cui è soggetta la specie a scala continentale (BirdLife International, 2004). La tendenza al declino dell'averla piccola è confermata dalla continua diminuzione della popolazione nidificante, con estinzione locale in aree potenzialmente idonee ad ospitare la specie (ad es. Alpe La Piana, sopra Colloro), e dalla contrazione dell'area di distribuzione (Fig. 28; Tab. 4).

\section{Corvidae}

Ghiandaia Garrulus glandarius SB

Specie comune nell'area di studio, segnalata fino a $1.500 \mathrm{~m}$ s.l.m. in periodo riproduttivo. Nel 1993 sono state rilevate 25 coppie su 115 punti di ascolto, con una dominanza relativa dell'1,6\% (Movalli \& Grimaldi, 1996).

Gazza (Pica pica) M

Una sola osservazione nota: 2 individui a Unchio il 13/03/2003 (FC).

\section{Nocciolaia (Nucifraga caryocatactes) SB}

Specie non comune e localizzata nell'area di studio in corrispondenza dei nuclei di conifere di origine naturale (pino uncinato, abete bianco) o artificiale (abete rosso), quali le località Cappella Porta (Caprezzo, massimo 3 individui il 20/03/2014: FC, LL), Cappella Fina-Monte Todum (4 individui il 22/03/2003 e 3 individui il 18/05/2013: FC), Pizzo Mottac (massimo 14 individui il 20/07/2012: MD). Movalli e Grimaldi (1996) segnalano la presenza nel 1993 di 9 coppie su 115 punti di ascolto, per una dominanza relativa dello $0,6 \%$.

In periodo non riproduttivo:

- 8 individui tra Rifugio Parpinasca e Colma di Basagrana il 28/08/2012 (FC e AG);

- singoli individui presso Alpe La Motta (Premosello C.) il 31/10/2010 (FC), presso l'Alpe Portaiola il 17/09/2013, tra Alpe La Motta e la Colma di Premosello il 5 e 28/08/2014 e presso l'Alpe Terza ad agosto-settembre 2014 (AM).

Si stima la presenza nell'area di studio di 10-20 coppie nidificanti.

\section{Gracchio alpino (Pyrrhocorax graculus) SB}

Il gracchio alpino è presente con alcune colonie negli ambienti rocciosi alle quote più elevate comprese nell'area di studio. Ventinove coppie erano state individuate nel 1993 su 115 punti d'ascolto, per una dominanza relativa dell'1,9\% (Movalli \& Grimaldi, 1996). In anni recenti si segnalano le seguenti osservazioni in periodo riproduttivo:

- Bocchetta di Terza: coppia che fa oggetto di mobbing un'aquila reale adulta l'11/07/2013 (FC, CM, ST), 10 il 5/07/2014 (LL, MP) e 9, tra i quali alcuni giovani, il 4/09/2014 (MP);

- Scaredi: 17 individui il 30/06/2014 (FC) e ancora 17 individui il 06/09/2014 sul limitrofo Cimone di Straolgio (AM);

- Colma di Premosello: 14 individui 1'11/06/2013 (FC, CM, PD) e sempre 14 individui l'8/07/2014 (LL, MP);

Tab. 4 - Territori di averla piccola nel Parco e aree limitrofe. / Redbacked Shrike territories in the Park and surrounding areas.

\begin{tabular}{|l|c|c|}
\hline Anno & Numero territori & Fonte \\
\hline 1993 & 6 & Movalli \& Grimaldi, 1996 \\
\hline 2009 & 4 & Casale \& Brambilla, 2010b \\
\hline 2013 & 1 & Casale, 2014 \\
\hline
\end{tabular}


- Pizzo Stagno: 13 individui il 30/06/14 (FC) e circa 25 individui il 25/07/2014 (DR);

- Rocce del Gridone: circa 15 individui il 14/08/2014 (FC, MB).

Massime concentrazioni post-riproduttive:

- 26 individui il 31/10/2010 presso la Colma di Premosello (FC);

- circa 55 individui presso il Pizzo Ragno il 13/09/2013 (FC, CM, MP);

- circa 30 individui presso la Bocchetta di Vald il 30/10/2013 (FC, DR);

- circa 40 individui presso la Testa di Menta il 12/09/2014 (LL, MP);

- circa 100 individui presso la Punta Tignolino l'8/09/2014 (BDVG);

- un gruppo di circa 100 individui osservato in più occasioni in periodo post-riproduttivo negli ultimi anni presso i Corni di Nibbio (AP).

Si stima la presenza nell'area di studio di 50-70 coppie.

Taccola (Corvus monedula) M irr

Due sole osservazioni note nell'area di studio:

- 5 individui verosimilmente in migrazione il
30/3/2011 sul versante sopra Capraga (RB);

- 1 individuo in migrazione sul Monte Carza il 5/05/2014 diretto verso N (FC, MP).

La taccola nidifica nei limitrofi centri abitati di Verbania Pallanza e Cannobio (Bionda \& Bordignon, 2006).

Cornacchia nera (Corvus corone) M, B irr

Pochi i dati noti di presenza: 1 giovane a Oggiogno (Cannero) il 23/06/2001 (CM), 1 individuo a Traffiume (Cannobio) il 27/07/2002 (FC e Enrico Giussani), 1 tra Cossogno e Ungiasca il 2/01/2013 (Giovanni Natale \& Maria Grazia Carpi sulla piattaforma on-line Ornitho.it), un individuo a Cambiasca l'8/05/2013 (FC).

\section{Cornacchia grigia (Corvus cornix) SB}

La scarsità di ambienti prativi a basse quote limita la presenza della specie nell'area di studio. Movalli e Grimaldi (1996) segnalano nel 1993 la presenza di una sola coppia nel Parco su 115 punti di ascolto. Nell'area di studio è verosimile che abbia nidificato con almeno due coppie nel 2013-2014: una nei pressi degli abitati di Trarego e Viggiona ed una a Cambiasca (FC). Altri dati di presenza in periodo riproduttivo:

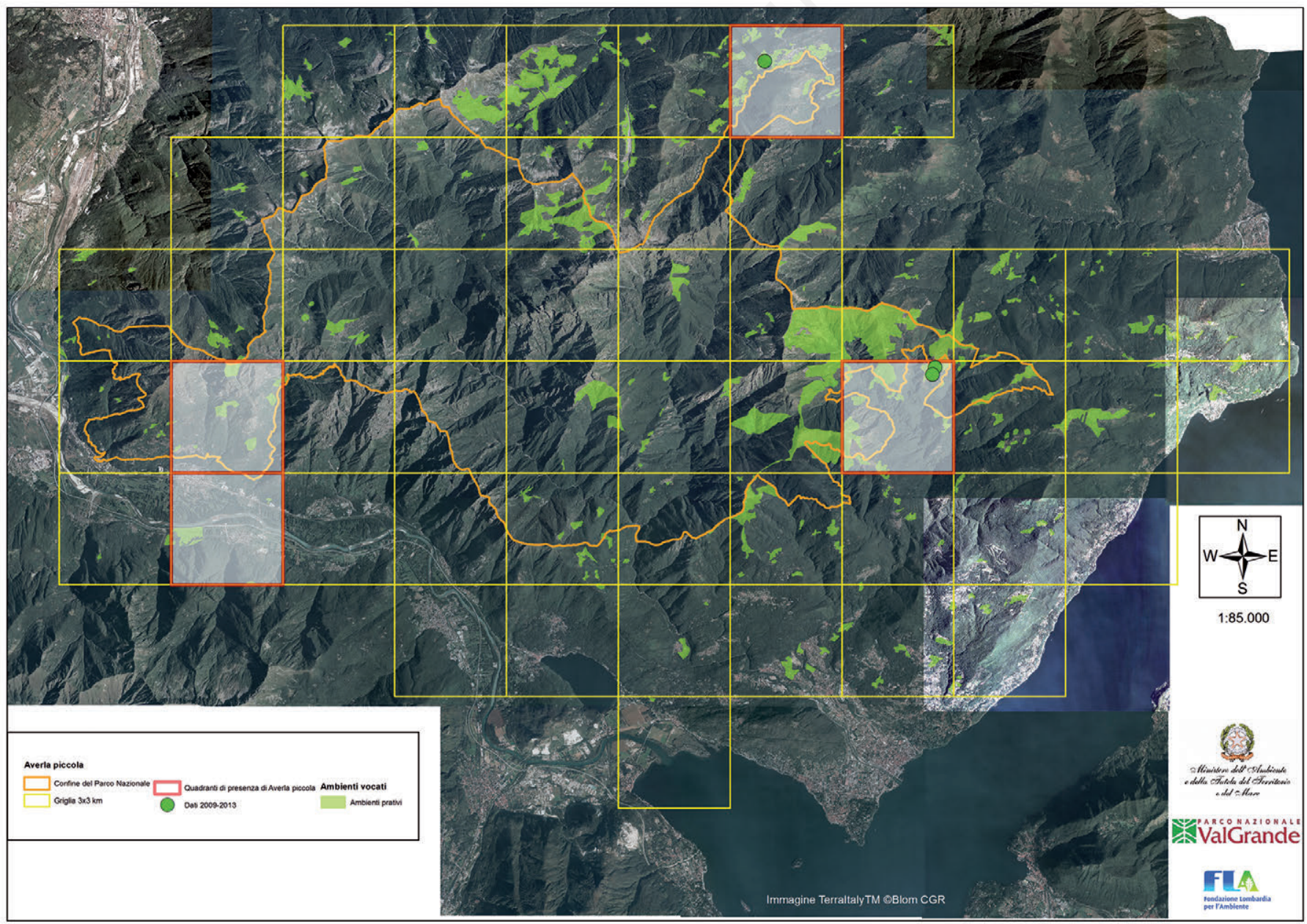

Fig. 28 - Averla piccola nel Parco Nazionale della Val Grande e aree limitrofe. Quadranti colorati: aree in cui la specie è stata rilevata in periodo riproduttivo nel 1993-2014; pallini verdi: dati di presenza in periodo riproduttivo nel 2009-2014. / Red-backed Shrike in Val Grande National Park and surrounding areas. Colored squares indicate were the species was recorded during the breeding season in 1993-2014 and green dots in 2009-2014. 
- 1 individuo a Aurano il 16/04/2000 (FC);

- 1 individuo il 24/03/2002 tra Ungiasca e Cossogno (FC);

- 1 individuo a Cossogno il 13/03/2003 (FC);

- una coppia a Traffiume (Cannobio) il 27/03/2002 (FC e Enrico Giussani);

- 1 individuo a Miazzina il 18/05/2013 (FC).

\section{Corvo imperiale (Corvus corax) SB}

La specie frequenta le quote più elevate dell'area di studio, in particolare gli ambienti prativi e rocciosi al di sopra del limite della vegetazione arborea. Nel periodo estivo sfrutta per fini trofici gli avanzi alimentari lasciati dagli escursionisti sulle vette o nei pressi di rifugi. Quattro territori erano stati rilevati nel 1993 su 115 punti d'ascolto da Movalli \& Grimaldi (1996), per una dominanza relativa dello $0,2 \%$. Nidificazione accertata: nido sotto la vetta del Monte Zeda il 05/07/2003 (CM). Alcune aggregazioni:

- roost di 43 individui il 4/04/2000 sopra Cosasca, collegabile alla limitrofa discarica di Domodossola (FC);

- 8 individui sopra l'Alpe La Motta (Premosello C.) il 31/10/2010 (FC);

- circa 15 individui al Pian Vadà il 6/04/2013 (RB, FC, CM, FCL, DF, PP, LP);

- 35 individui il 9/08/2013 nell'area di Scaredi per la presenza dei corpi di 66 pecore uccise da un fulmine (MP, FCA, MD, ST).

Si stima la presenza nell'area di studio di 10-20 coppie nidificanti.

\section{Sturnidae}

Storno (Sturnus vulgaris) SB, M reg, W irr

La scarsità di ambienti prativi a basse quote limita la presenza della specie nell'area di studio, che nidifica negli abitati di Vogogna, Cambiasca (un nido il 13/06/2001: FC) e forse in pochi altri villaggi (1 adulto a Unchio il 5/05/2001 e il 6/03/2003: FC). Nessun territorio era stato rilevato nel Parco nel 1993 su 115 punti d'ascolto (Movalli \& Grimaldi, 1996).

\section{Passeridae}

\section{Passera europea (Passer domesticus) SB}

La popolazione italiae della passera europea (la cui posizione sistematica non è ancora chiarita secondo Fracasso et al., 2009) nidifica in molti centri abitati compresi nell'area di studio: Vogogna, Colloro, Cicogna, Rovegro, Cossogno, Miazzina, Intragna, Caprezzo (2 maschi il 22/04/1213: FC), Aurano (adulto che trasporta materiale il 16/04/2000: FC), Finero, Falmenta (circa 15 individui e 3 nidi con pulcini il 5/06/2014: FC), Gurro (almeno 5 individui il 5/06/2014, con trasporto dell'imbeccata, FC). 28 coppie individuate nel $1993 \mathrm{su}$ 115 punti d'ascolto per una dominanza relativa dello 0,2\% (Movalli \& Grimaldi, 1996).

La presenza di individui appartenenti alla sottospecie nominale è stata segnalata a Cicogna (maschi in canto il 31/3/2001: RB) e a Falmenta (individuo con imbeccata il 29/06/2001: RB).
Si stima la presenza di 50-100 coppie nidificanti della popolazione italiae e di poche coppie della popolazione continentale.

\section{Passera mattugia (Passer montanus) SB}

Specie non comune e localizzata come nidificante, soprattutto in prossimità di piccoli centri abitati. La specie viene segnalata nel 1993 con 3 coppie (a Colloro il 20/06) su 115 punti di ascolto da Movalli \& Grimaldi (1996). Pochi i dati in periodo riproduttivo:

- un giovane a Intragna il 18/05/2000 (FC);

- 1 individuo il 7/06/2002 a Unchio (FC);

- alcuni individui (solo canto) a Piancassone (Cannero) il 24/05/2014 (FC).

Si stima la presenza di 5-10 coppie nidificanti nell'area di studio.

\section{Fringillidae}

Fringuello (Fringilla coelebs) SB, M reg

Specie molto comune, è la più abbondante nell'area di studio, come emerso dallo studio di Movalli e Grimaldi (1996), con 190 territori individuati nel 1993 su 115 punti d'ascolto, per una dominanza relativa del $12,4 \%$. In periodo riproduttivo: nido con adulto in cova in siepe di Prunus laurocerasus a Cossogno il 27/04/2003 e nido con pulcini nel medesimo sito il 24/05/2004 (FC); massima densità rilevata di 28 individui in canto nella pecceta tra Cappella Porta e Rifugio Pian Cavallone 1'8/05/13, pari a 14 territori/km (FC).

In migrazione: 104 individui a Scaredi il 18/10/2013, 17 individui alla Colma di Premosello il 25/10/2013 e 2 individui alla Bocchetta di Cavalla il 30/10/2013 (Casale, 2014).

Si stima la presenza di 500-600 coppie nidificanti nell'area di studio.

Peppola (Fringilla montifringilla) M reg, W irr

L'area di studio è interessata dalla presenza della specie durante le migrazioni (ad es. 9 individui a Scaredi il 25/10/2012, diretti a S: FC). In inverno vi è assai scarsa, ma dal 20/02/2005 al 7/04/2005 il versante meridionale della Val Vigezzo è stato interessato dalla presenza di un roost invernale di grandissime dimensioni, la cui consistenza è stata stimata, tramite conteggi fotografici, in 1,5-3 milioni di individui (Roberto Lardelli in G.P.S.O., 2007).

\section{Verzellino (Serinus serinus) SB, M reg}

Il verzellino nidifica in alcuni centri abitati compresi nell'area di studio. Nessun territorio era stato rilevato nel Parco negli anni '90 su 115 punti d'ascolto (Movalli \& Grimaldi, 1996). Alcuni dati in periodo riproduttivo:

- 1 individuo in canto il 13/07/2001 a Cossogno (FC);

- 1 individuo in canto a Cambiasca l'1/05/2002 (FC);

- 1 individuo in canto a Viggiona 1'8/05/2013 (FC);

- coppia a Miazzina il 18/05/2013 (FC);

- coppia a Cicogna il 31/05/2013 (FC).

Si stima la presenza di 10-20 coppie nidificanti nell'area di studio.

In migrazione sul Monte Carza (Trarego-Viggiona): 
- 1 individuo in sorvolo verso S il 17/04/2014 (FC, $\mathrm{CM}$;

- 1 individuo il 17/05/2014 (FC, MP).

Inoltre, in transito verso $\mathrm{S}$ a Scaredi, 4 individui il $18 / 10 / 2013$ (Casale, 2014) e 6 individui il 17/10/2014 (FC, MP).

\section{Verdone (Carduelis chloris) SB}

Nidifica in pochi centri abitati compresi nell'area di studio, ad esempio a Premeno ( 1 individuo in canto il 25/06/2013: FC). Nessun territorio era stato rilevato nel Parco negli anni '90 su 115 punti d'ascolto (Movalli \& Grimaldi, 1996).

Si stima la presenza di 5-10 coppie nidificanti nell'area di studio.

\section{Cardellino (Carduelis carduelis) SB, M reg}

Nidifica in pochi centri abitati compresi nell'area di studio (ad esempio, una coppia il 25/06/2002 a Cossogno: FC). Nessun territorio era stato rilevato nel Parco nel 1993 su 115 punti d'ascolto (Movalli \& Grimaldi, 1996).

Si stima la presenza di 5-10 coppie nidificanti nell'area di studio.

In migrazione:

- circa 15 individui in alta Val Loana il 30/10/1996 (FC);

- 7 individui verso $S$ a Scaredi il 18/10/2013 e 2 individui verso $\mathrm{S}$ alla Colma di Premosello il 25/10/2013 (Casale, 2014);

- 10 individui verso S a Scaredi il 17/10/2014 (FC, MP).

\section{Venturone alpino (Serinus citrinella) M irr}

La specie è stata scarsamente rilevata nell'area di studio durante le migrazioni. Sono noti i seguenti dati di presenza:

- 1 individuo in transito il 9/05/2000 sulla Cima del Morissolo (1.350 m) (Bionda \& Bordignon, 2006);

- 3 individui il 10/10/2010 a $2.050 \mathrm{~m}$ s.l.m., lungo la cresta tra Monte Zeda e Pizzo della Marona (FC);

- 1 individuo in transito il 18/10/2013 presso Scaredi (FC);

- 1 individuo il 25/08/2014 lungo la dorsale tra Monte Todano e Il Pizzo (FC, LL);

- 1 individuo il 16/09/2014 tra Alpe Stavelli e la Colma di Premosello (FC);

- 1 individuo diretto a $\mathrm{S}$ alla Colma di Premosello 1'8/10/2014 (FC, CM);

- 1 individuo diretto a W e 1 diretto a SE a Scaredi il 17/10/2014 (FC, MP).

Lucherino (Carduelis spinus) M reg, B ?, W reg

Specie comune durante le migrazioni nell'area di studio, mentre vi è rara e localizzata durante il periodo riproduttivo, in particolare in corrispondenza delle seguenti località:

- boschi di conifere localizzati tra Cappella Porta (Caprezzo) e Rifugio Pian Cavallone (1 1'8/05/2013: FC);

- tra Cappella Fina (Miazzina) e Monte Todum;

- in loc. Pian dei Sali (Malesco, 1 il 23/05/2009: FC);
_ in località Biogna (Aurano, 1 il 21/07/2009: Casale \& Brambilla, 2010b)

La specie non era stata censita a metà anni ' 90 nel Parco da Movalli \& Grimaldi (1996) su 115 punti d'ascolto.

In periodo non riproduttivo:

- circa 15 individui a Cossogno il 28/09/2001 (FC);

- 12 individui presso l'Istituto Auxologico di Piancavallo il 20/03/2002 (FC);

- 3 individui il 23/03/2002 a Pian di Sole (Premeno; FC);

- alcuni sul Monterosso il 23/03/2002 (Verbania; FC);

- circa 15 individui il 31/10/10 alla Colma di Premosello (FC);

- 34 individui in transito da Scaredi il 18/10/2013, 29 dalla Colma di Premosello il 25/10/13 e 7 individui dalla Bocchetta di Vald il 30/10/2013 (Casale, 2014);

- circa 25 individui tra Alpe Bondolo e Bocchetta di Vald il 30/10/2013 (FC, DR);

- 1 individuo tra Alpe Pont e Cappella Porta e 1 individuo a Cappella Porta (Caprezzo) il 6/03/2014 (FC);

- 1 individuo a Cappella Porta il 20/03/2014 (FC, LL);

- 2 individui in volo verso $\mathrm{N}$ sulla dorsale Pizzo Pernice-Monte Todano 1'8/05/2014 (FC);

- 22 individui in transito a Scaredi il 17/10/2014 (FC, MP).

Fanello (Carduelis cannabina) M reg, B

Specie scarsa come nidificante nell'area di studio (sulla dorsale tra Monte Todano e Il Pizzo un maschio 1'8/05/2013 e un maschio 1'8/05/2014: FC). 2 territori su 115 punti d'ascolto, entrambi rilevati il 24/05/1993 presso l'Alpe Belmello (Movalli \& Grimaldi, 1996). In migrazione:

- circa 20 individui a Fondo Li Gabbi, in Val Loana, il 27/10/1996 e ancora il 30/10/1996 (FC);

- 2 individui il 23/03/2002 e 1 individuo il 03/04/2002 a Cossogno (FC);

- 1 individuo all'Alpe In La Piana il 9/04/2012 (BDVG);

- 3 individui in transito a Scaredi il 18/10/2013 e 4 individui alla Colma di Premosello il 25/10/2013 (Casale, 2014);

- 14 individui diretti a S alla Colma di Premosello il 3/10/2014 (FC, CM);

- 1 individuo il 17/04/2014 (FC, CM) e 2 individui il 5/05/2014 sul Monte Carza diretti a N (FC, MP).

Si stima la presenza di 1-5 coppie nidificanti nell'area di studio.

Organetto (Carduelis flammea) M reg, B

Specie scarsa come nidificante nell'area di studio (1-5 coppie stimate). Due territori su 115 punti d'ascolto, entrambi il 31/05/1993 presso l'Alpe Terza (a $1.560 \mathrm{~m}$ s.l.m.) e presso la Bocchetta di Terza (a $1.800 \mathrm{~m}$ ), in Val Pogallo (Movalli \& Grimaldi 1996). Negli anni successivi sono stati rilevati 1-3 territori:

- il 17/6/2000 un maschio in canto presso Scaredi (RB); 
- 1 individuo presso La Balma il 16/07/2009 (Brambilla \& Casale, 2010b);

- 1-2 territori rilevati 1'11/07/2014 tra Rifugio Parpinasca e Colma di Basagrana (FC, FCA, MD, DR), dove un individuo era stato osservato anche il 28/08/2012 (FC e AG).

Inoltre 2 individui in transito presso Bocchetta di Campo il 13/11/2007 (RB).

\section{Crociere (Loxia curvirostra) SB, M reg, W reg}

Specie localizzata nell'area di studio, legata alle poche aree con boschi naturali o artificiali di conifere. Alcuni dati:

- almeno 2 individui il 30/06/2002 sul Monte Todum e almeno 1 il 21/09/2002 in un rimboschimento di abeti rossi tra Cappella Fina e Monte Todum (FC);

- alcuni individui presso l'Alpe Pala (Miazzina) il 07/02/2002 (FC);

- 5 all'Alpe Nancino di Sopra il 28/09/2011 (BDVG);

- 1 individuo tra Rifugio Parpinasca e Colma di Basagrana il 28/08/2012 (FC e AG);

- 4-5 individui nella pecceta a monte di Cappella Porta il 22/04/2013 (FC, CM);

- 1 individuo alla Colma di Premosello 1'11/06/2013 (FC, LL, CM, PD);

- 17 individui in transito verso $\mathrm{N}$ al Passo Folungo il 25/06/2013 (FC);

- una femmina il 24/05/2014 diretta a S sul Monte Carza (FC, MP);

- 2 individui diretti a S a Scaredi il17/10/2014 (FC, MP).

Si segnala altresì il passaggio presso i colli alpini di gruppi diretti verso $\mathrm{N}$ con volo direzionale in periodo autunnale, fenomeno che potrebbe essere legato all'erratismo che caratterizza tale specie: 10 individui in transito a Scaredi il 18/10/2013, 4 individui in transito alla Colma di Premosello il 25/10/2013 (Casale 2014) e 4 individui nella medesima località l'8/10/2014 (FC, CM).

\section{Ciuffolotto (Pyrrhula pyrrhula) SB}

Specie legata a boschi freschi e ombrosi, è stata rilevata nel 1993 la presenza di 7 territori su 115 punti d'ascolto, per un indice di densità dello $0,4 \%$ (Movalli \& Grimaldi, 1996). Massima densità: 3 territori in alta Val Loana, tra Fondo Li Gabbi e Alpe Cortenuovo il 24/07/1994, pari a 1,1 territori/km (FC e Giuliano Tallone). Alcuni dati di presenza in periodo riproduttivo:

- 1 adulto e 1 giovane a Provola il 25/06/2000 (CM);

- 2 individui a Cappella Fina il 18/05/2013 (FC);

- 1 individuo tra Cappella Fina e Rifugio Pian Cavallone 1'8/05/2014 (FC);

- 1 individuo presso l'Alpe Boschelli il 3/07/2013 (FC, CM).

Si stima la presenza di 20-30 coppie nidificanti nell'area di studio.

Un maschio di Ciuffolotto di Komi (Pyrrhula pyrrhula pyrrhula) è stato osservato il 7/11/2014 nei pressi della Colma di Premosello (FC, CM). L'individuo era posato su un arbusto e ha emesso in più occasioni il tipico verso, permettendo di accertarne l'identificazione. Successivamente si è involato e ha svalicato la Colma, diretto verso $\mathrm{S}$.
Frosone (Coccothraustes coccothraustes) M reg, B, W reg Raro e localizzato come nidificante nell'area di studio. Alcuni dati in periodo riproduttivo:

- una coppia presso In La Piana a metà anni '90 (CM), confermata nel 2013 (4/07/2013: FC, CM) e nel 2014 (3/07/2014: LL, MP);

- 1 individuo il 21/12/2001 a Bieno (S. Bernardino V.: FC);

- 1 individuo il 7/08/2002 all'Alpe Cavallotti (FC);

- 1 giovane 1'1/07/2009 nella Valle di Finero (Casale \& Brambilla, 2010b).

In migrazione:

- 3 individui il 23/03/2002 a Pian di Sole (Premeno: FC, Enrico e Luca Giussani);

- 1 individuo presso l'Alpe La Piana (Premosello C.) il 2/04/2011 (BDVG);

- 10 individui diretti a S a Scaredi il 18/10/2013 (Casale, 2014);

- 5 individui all'Alpe Cortenuovo, in alta Val Loana, diretti verso S l'8/09/2014 (FC, MP);

- 10 individui tra Alpe La Motta e Alpe Stavelli il 3/10/2014 (FC, CM);

- 10 individui diretti a N a Scaredi il 17/10/2014 (FC, MP).

Si stima la presenza di 2-5 coppie nidificanti nell'area di studio.

\section{Emberizidae}

Zigolo giallo (Emberiza citrinella) M reg, $\mathrm{B}$

Specie localizzata in pochi siti compresi nell'area di studio, per lo più in aree ben soleggiate, di margine tra ambienti prativi e boschivi. Movalli \& Grimaldi (1996) segnalano nel 1993 un territorio su 115 punti di ascolto effettuati nell'area protetta. Successivamente, 1 maschio è stato rilevato il 30/6/1996 presso l'Alpe Basso (CM) e 2 maschi il 18/05/2000 rispettivamente in località Pian d'Arla (Aurano) e in località Sunfaì, in comune di Intragna (FC). Lo studio realizzato nel 2009 sugli uccelli nidificanti negli ambienti aperti ne ha confermato la presenza con le seguenti osservazioni: 2 maschi in canto a Dalia, nei pressi di Finero, 1'1/07/2009 e 1 maschio in canto a Passo Folungo il 22/07/2009 (Casale \& Brambilla, 2010b). Successivamente, sono noti i seguenti dati:

- 1 maschio in canto sul Monte Bavarione il 25/06/2013 (FC);

- 2 maschi in località Pian d'Arla (Aurano) il $15 / 06 / 2014$, uno in canto e uno allarmato (FC);

- 1 maschio in canto nella Valle del Basso nella prima metà di luglio 2014 (LL).

In migrazione:

- 1 individuo il 27/03/2014 in sorvolo sul Monte Carza, diretto a $\mathrm{N}$ (FC);

- 6 individui diretti a $\mathrm{N}$ alla Colma di Premosello 1'8/10/2014 (FC, CM).

Si stima la presenza di 5-10 coppie nidificanti nell'area di studio.

\section{Zigolo muciatto (Emberiza cia) SB}

Dai risultati emersi dallo studio sull'avifauna nidificante negli ambienti aperti del Parco, condotto nel 2009, lo zigolo muciatto è risultato essere di gran lunga il Passeriforme più 
diffuso negli ambienti oggetto di indagine, con complessivi 44 territori all'interno delle 4 aree campione indagate, con densità che nell'area di Colloro raggiungono 3 coppie/10 ha (Casale \& Brambilla, 2010b). In inverno, lo zigolo muciatto scende a basse quote: 1 individuo il 21/12/2001 e 2 individui il 6/01/2004 nel paese di Cossogno (FC), alcuni all'Alpe Pala (Miazzina) il 07/02/2002 (FC). I territori individuati nel 1993 erano 33 su 115 punti d'ascolto, per una dominanza relativa di 2,1\% (Movalli \& Grimaldi, 1996).

Diversamente da altre specie, da un raffronto tra i dati emersi durante i punti d'ascolto per il "Monitoraggio della biodiversità", il numero dei territori è confrontabile sia nel mese di giugno (6 nel 2013 e 4 nel 2014) che nel mese di luglio (11 nel 2013 e 12 nel 2014), verosimilmente in quanto la specie è legata ad ambienti esposti a meridione, tra i primi a essere liberi dalla neve anche in situazione di forte innevamento invernale.

In periodo riproduttivo:

- 1 individuo con imbeccata il 22/6/1996 a Caseracce $(\mathrm{CM})$;

- 1 individuo con imbeccata il 21/06/2012 e un giovane da poco involato il 25/06/2013 nella brughiera sopra Biogna (FC);

- coppia con giovani da poco involati all'Alpe Terza, in Val Pogallo, 1'11/07/2013 (FC, CM);

- 1 giovane alle pendici dela Cima Morissolo il 22/08/2013 (FC);

- 2 giovani con un adulto il 5/06/2014 sul Monte Carza (FC, MP).

Si stima la presenza di 100-200 coppie nidificanti nell'area di studio.

\section{MONITORAGGIO DELLA BIODIVERSITÀ IN AMBIENTE ALPINO}

Nel corso delle stagioni riproduttive 2013 e 2014 il Parco Nazionale della Val Grande ha aderito al progetto "Monitoraggio della biodiversità in ambiente alpino" realizzato in collaborazione con altri Parchi Nazionali alpini italiani. La metodologia standard prevedeva di individuare alcuni transetti localizzati lungo gradienti altitudinali tali per cui tutti gli ambienti più rappresentativi dell'area protetta venissero percorsi . Una volta individuati i 3 transetti all'interno del Parco, sono state localizzate delle stazioni (plot) ogni 200 metri di altitudine (per un totale di 18 stazioni), in corrispondenza delle quali effettuare indagini su vari aspetti della flora e diversi taxa animali ivi presenti. Per quanto riguarda l'avifauna nidificante sono stati effettuati, in corrispondenza di ogni stazione, due punti di ascolto (uno a giugno e uno a luglio) in corrispondenza

Tab. 5 - Transetti oggetto di monitoraggio della biodiversità nel $2013 \mathrm{e}$ 2014. / Transects that were monitored in 2013 and 2014.

\begin{tabular}{|l|l|}
\hline Codice transetto & Transetto \\
\hline T01 & Da sopra Colloro a Colma di Premosello \\
\hline T02 & Da In La Piana a Scaredi \\
\hline T03 & Da Pogallo a Bocchetta di Terza \\
\hline
\end{tabular}

dei quali venivano rilevati tutti gli individui contattati (osservati o uditi) entro $100 \mathrm{~m}$, tra 100 e $500 \mathrm{~m}$ e oltre 500 m. I 3 transetti individuati sono indicati in Tab. 5.

In Tab. 6 viene fornito l'elenco delle 52 specie rilevate nei punti d'ascolto, sommando i dati relativi ai tre intervalli (0-100 m, 100-500 m, oltre $500 \mathrm{~m})$.

La specie dominante è risultata essere il fringuello (12,2\% degli individui), confermando quanto rilevato da Movalli \& Grimaldi (1996) negli anni '90. A seguire, le specie più diffuse sono risultate essere capinera, scricciolo, pettirosso e merlo, con valori compresi tra 4,9 e $5,0 \%$. Complessivamente queste 5 specie costituiscono il $31,9 \%$ degli individui rilevati.

\section{MIGRAZIONE PRIMAVERILE DEI RAPACI DIURNI}

Lo studio della migrazione dei rapaci in Italia, effettuato con metodi standardizzati, ebbe inizio nella seconda metà degli anni ' 80 del XX secolo. Attualmente i siti che vengono monitorati con continuità sono 13 , in un panorama composto da almeno 32 località interessate, delle quali meno di 10 lungo l'arco alpino, localizzate soprattutto nelle valli del Piemonte occidentale, sulla sponda occidentale del Lago di Garda (Cima Comér), in Trentino e in Alto-Adige (Fig. 29). Oggi conosciamo a

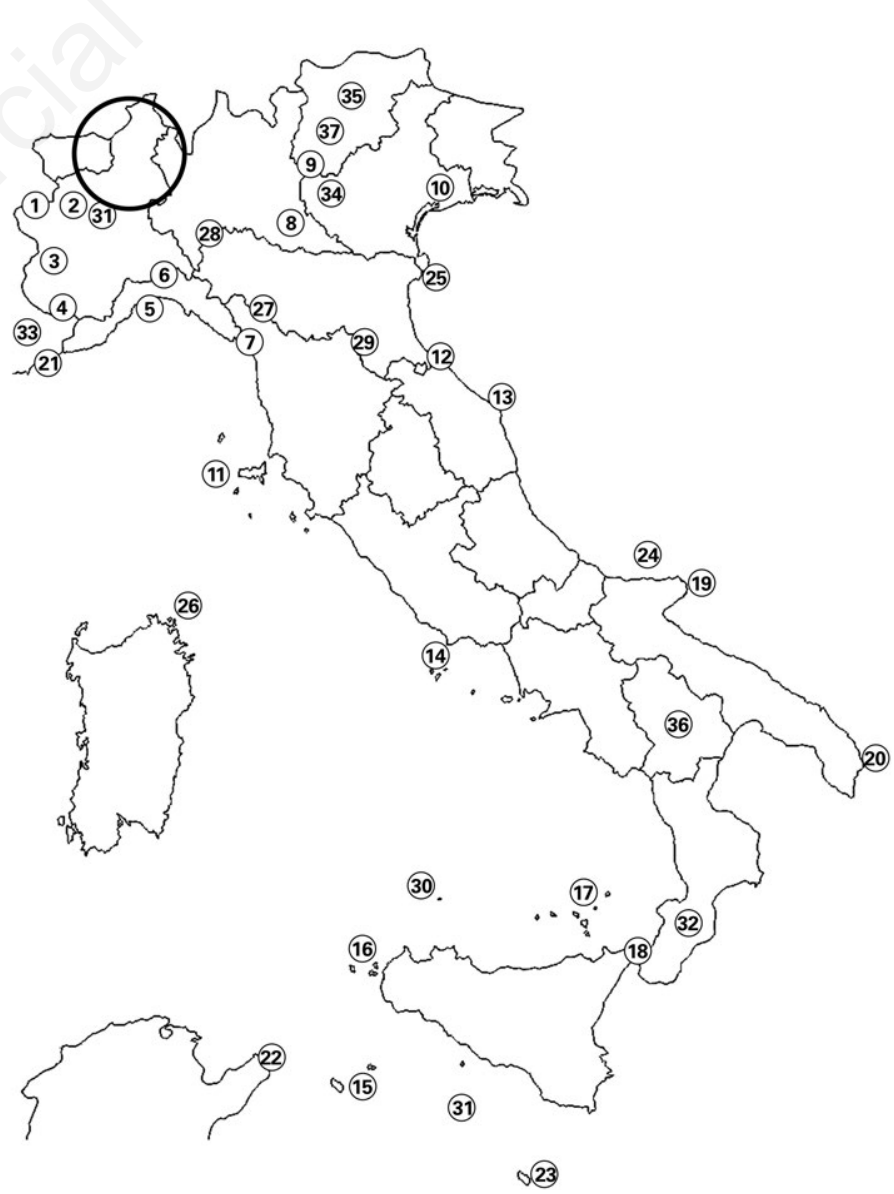

Fig. 29 - Siti di osservazione della migrazione dei rapaci in Italia. Viene evidenziata la localizzazione dell'area indagata. / Sites for the observation of raptor migration in Italy. The location of the study area is highlighted (da / from: Infomigrans, 2011). 
Tab. 6 - Elenco delle specie rilevate nei 18 punti d'ascolto distribuiti lungo i transetti e valore medio degli individui complessivamente censiti nel 2013 e 2014. Tra i valori rilevati nei mesi di giugno e luglio è stato considerato il valore più elevato. / List of recorded species during 18 point counts located along the transects and mean value of individuals overall censused in 2013 and 2014. Between the values recorded in June and July, it was selected the highest one.

\begin{tabular}{|c|c|c|}
\hline Nome scientifico & $\begin{array}{c}\mathrm{N} \text { ind. valore medio } \\
2013-2014\end{array}$ & $\%$ \\
\hline Tetrao tetrix & 4 & 0,9 \\
\hline Alectoris graeca & 2 & 0,4 \\
\hline Circaetus gallicus & 1 & 0,2 \\
\hline Aquila chrysaetos & 5 & 1,0 \\
\hline Accipiter gentilis & 0,5 & 0,1 \\
\hline Accipiter nisus & 0,5 & 0,1 \\
\hline Buteo buteo & 0,5 & 0,1 \\
\hline Falco tinnunculus & 5 & 1,0 \\
\hline Cuculus canorus & 13,5 & 3,0 \\
\hline Stryx aluco & 3 & 0,6 \\
\hline Apus apus & 47 & 10,1 \\
\hline Apus melba & 3,5 & 0,7 \\
\hline Picus viridis & 0,5 & 0,1 \\
\hline Dryocopus martius & 5,5 & 1,2 \\
\hline Dendrocopos major & 3 & 0,6 \\
\hline Ptyonoprogne rupestris & 6 & 1,2 \\
\hline Anthus trivialis & 21,5 & 4,7 \\
\hline Anthus spinoletta & 8 & 1,7 \\
\hline Motacilla cinerea & 3 & 0,6 \\
\hline Cinclus cinclus & 0,5 & 0,1 \\
\hline Troglodytes troglodytes & 22,5 & 4,9 \\
\hline Prunella modularis & 8,5 & 1,8 \\
\hline Prunella collaris & 3,5 & 0,7 \\
\hline Eritachus rubecola & 22,5 & 4,9 \\
\hline Phoenicurus ochruros & 9 & 1,9 \\
\hline Phoenicurus phoenicurus & 0,5 & 0,1 \\
\hline Oenanthe oenanthe & 1 & 0,2 \\
\hline Turdus merula & 22,5 & 4,9 \\
\hline Turdus philomelos & 12 & 2,6 \\
\hline Turdus viscivorus & 7,5 & 1,6 \\
\hline Sylvia atricapilla & 23,5 & 5,0 \\
\hline Sylvia borin & 9 & 1,9 \\
\hline Sylvia curruca & 5 & 1,0 \\
\hline Phylloscopus bonelli & 1,5 & 0,3 \\
\hline Phylloscopus collybita & 13,5 & 3,0 \\
\hline Aegithalos caudatus & 17 & 3,6 \\
\hline Cyanistes caeruleus & 11 & 2,3 \\
\hline Parus major & 9 & 1,9 \\
\hline Periparus ater & 2,5 & 0,6 \\
\hline Poecile palustris & 9,5 & 2,0 \\
\hline Sitta europaea & 3 & 0,6 \\
\hline Tichodroma muraria & 0,5 & 0,1 \\
\hline Certhia familiaris & 2 & 0,4 \\
\hline Certhia brachydactyla & 1,5 & 0,3 \\
\hline Garrulus glandarius & 8,5 & 1,8 \\
\hline Pyrrhocorax graculus & 21,5 & 4,7 \\
\hline Corvus corax & 7 & 1,6 \\
\hline Fringilla coelebs & 56,5 & 12,2 \\
\hline Loxia curvirostra & 6 & 1,2 \\
\hline Pyrrhula pyrrhula & 1,5 & 0,3 \\
\hline Coccothraustes coccothraustes & 2 & 0,4 \\
\hline Emberiza cia & 12,5 & 2,8 \\
\hline Totale & 467 & 100 \\
\hline
\end{tabular}

grandi linee le rotte di migrazione, i periodi di passo e una stima degli effettivi migranti della maggior parte delle specie, soprattutto veleggiatrici, anche se le conoscenze rimangono ancora lacunose in alcune aree intermedie fra $\mathrm{i}$ siti principali (Giraudo, 2007).

In Piemonte, lo studio della migrazione visiva (visible migration), in particolare per quanto riguarda il settore alpino, è iniziata a partire dalla fine degli anni ' 80 del secolo scorso con osservazioni standardizzate su alcuni valichi o valli interessati da particolari concentrazioni di uccelli durante i movimenti migratori (Reteuna, 1994; Toffoli \& Bellone, 1996; Giraudo \& Toffoli, 2003).

Come emerge dalla mappa (Fig. 29), non rientra tra i settori oggetto di tale tipologia di monitoraggio l'area del Piemonte orientale e della Lombardia occidentale, e quindi in particolare la linea migratoria che segue il corso del fiume Ticino in territorio italiano (Bovio, 1994; Fornasari, 2003; Calvi et al., 2011; Casale et al., 2014) e che, proseguendo verso nord, percorre il Lago Maggiore (Ente Parchi e riserve naturali del Lago Maggiore, 2007) e quindi il corso del Ticino in territorio elvetico.

Nel corso delle primavere 2013 e 2014 è stato condotto uno studio preliminare sulla migrazione dei rapaci diurni nel Parco Nazionale della Val Grande e in aree limitrofe, in particolare lungo la linea migratoria che, da Sud a Nord, segue il corso del fiume Ticino e del Lago Maggiore (Fig. 30) per poi scavalcare le Alpi. L'attenzione è stata focalizzata sulla migrazione pre-nuziale in quanto è quella che interessa le Alpi con flussi più consistenti, mentre, per quanto concerne quella post-nuziale, vari studi hanno evidenziato la presenza di un flusso migratorio che dopo aver attraversato su ampio fronte l'Europa centrale, giunto in

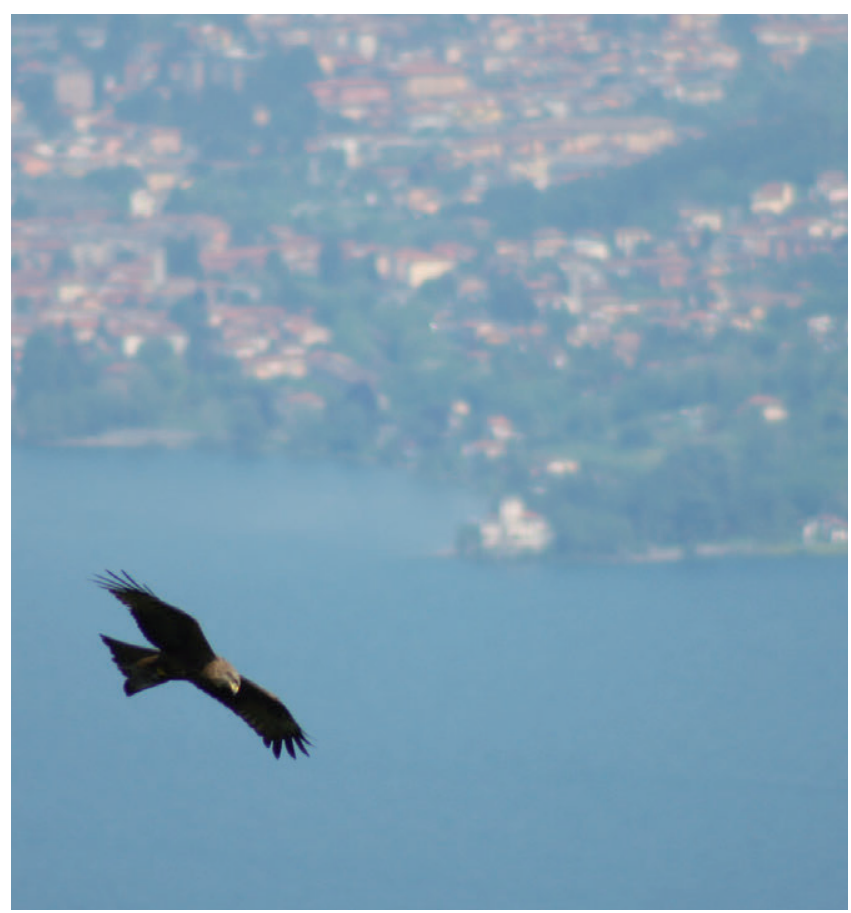

Fig. 30 - Nibbio bruno in volteggio. Sullo sfondo, il Lago Maggiore e la riva lombarda. / A soaring Black Kite. On the back: Lake Maggiore and the Lombardy shore. (Foto / Photo Fabio Casale). 
prossimità delle Alpi tende ad evitarle, piega sensibilmente verso occidente lungo i contrafforti settentrionali più esterni, dando luogo così ad un transito prevalente verso Ovest. Solo una minima parte degli uccelli sembra infatti attraversare le Alpi con direzione meridionale, e ciò avviene soprattutto al sopraggiungere di perturbazioni atlantiche solitamente accompagnate da venti occidentali che, spirando contrari alla direzione di volo, costringono i migratori a piegare verso le valli alpine e quindi a penetrare nelle Alpi (Bruderer, 1996; Bruderer \& Jenni, 1990). Le indagini di campo sono state condotte nei mesi di marzo, aprile e maggio e, trattandosi di un argomento finora mai studiato nell'area del Verbano, si è dapprima proceduto a:

- individuare le aree maggiormente interessate dal flusso migratorio;

- individuare i punti dai quali fosse possibile osservare il maggior numero di individui;

- effettuare una prima analisi quali-quantitativa del flusso migratorio.

Da tali indagini preliminari, è stato possibile definire un'individuazione di massima di tre rotte migratorie principali (Tab. 7).

Delle tre rotte in Tab. 7, l'indagine si è successivamente focalizzata sulle prime due, in quanto ricadenti nella più

Tab. 7 - Principali rotte migratorie primaverili per rapaci diurni nell'area di studio. / Main spring migratory flyways for raptors in the study area.

\begin{tabular}{|l|c|}
\hline Rotta migratoria & Dentro i confini del Parco \\
\hline Linea del Verbano (dorsale Cresta della Ciresa-Cima di Morissolo-Monte Carza) & No \\
\hline $\begin{array}{l}\text { Linea Pizzo Pernice-Monte Todano (dorsale Monte Todum-Pizzo Pernice-Rifugio } \\
\text { Pian Cavallone-Monte Todano) }\end{array}$ & $\mathrm{Si}$ \\
\hline $\begin{array}{l}\text { Linea del fiume Toce (lungo la dorsale e i versanti che delimitano i settori meridionale } \\
\text { e occidentale del Parco, seguendo il percorso della Val d'Ossola) }\end{array}$ & In parte \\
\hline
\end{tabular}

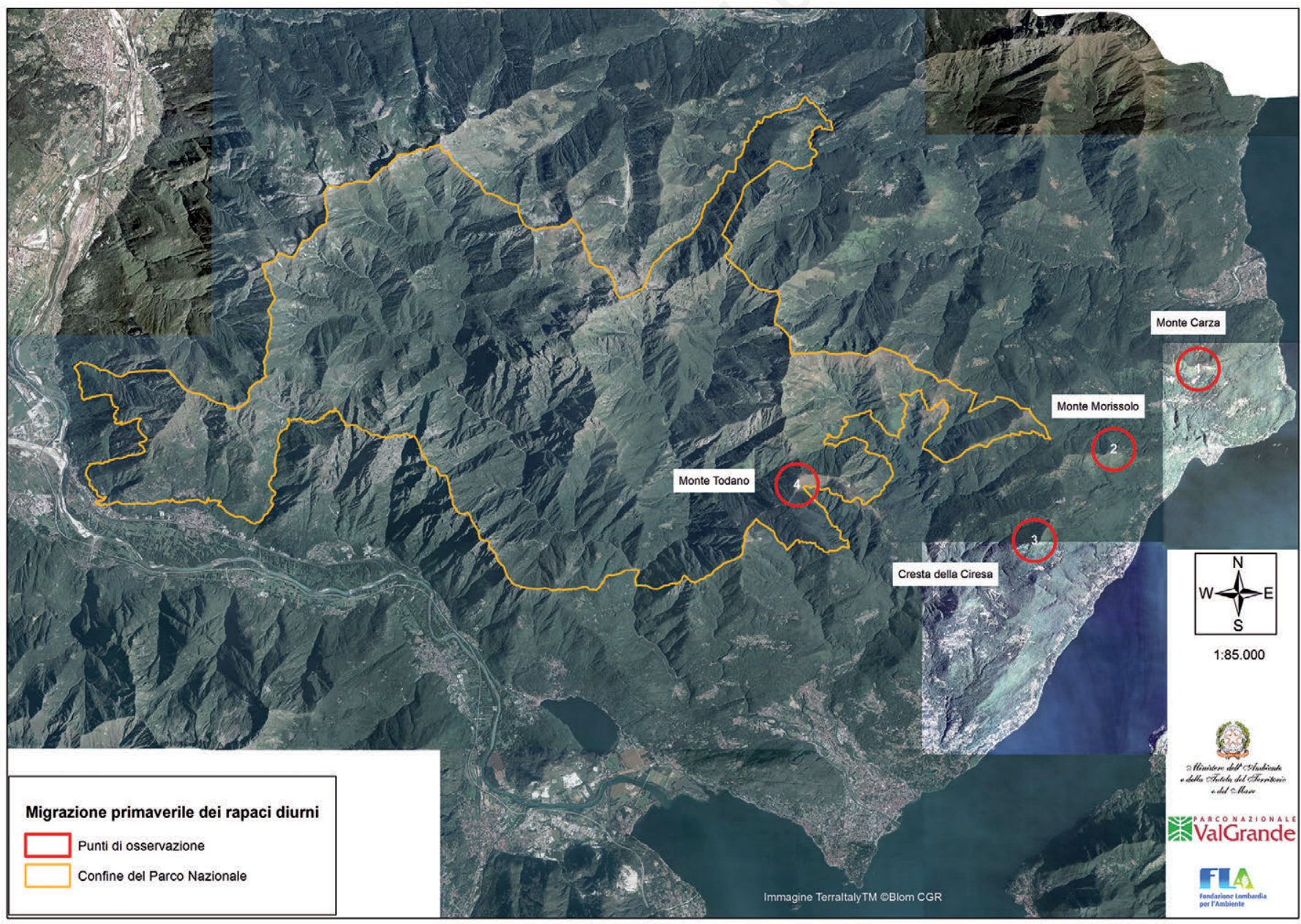

Fig. 31 - Punti più vocati a svolgere censimenti dei rapaci durante la migrazione primaverile. / Most suitable points for monitoring spring migration of raptors. 
vasta linea di migrazione che percorre Ticino e Lago Maggiore, mentre la terza è verosimile che segua il corso della val d'Ossola e del fiume Toce (Bionda, 2005) e, inoltre, in quanto interessate da un flusso numericamente più consistente di individui. I punti di osservazione localizzati lungo tali rotte vengono indicati in Fig. 31. Il Monte Carza, in particolare, è emerso essere il punto di osservazione più favorevole, probabilmente in quanto è collocato nel punto in cui il Lago Maggiore si restringe maggiormente e inoltre curva leggermente verso est. Tale altura rappresenta un punto di riferimento per i rapaci che seguono la riva occidentale del Verbano oppure giungono in volo sopra il lago, nonché, come osservato in più occasioni durante il monitoraggio, l'ultima possibilità di approfittare di una corrente ascensionale ("termica") prima di procedere verso il settore settentrionale del Verbano (Figg. 30 e 32).

I censimenti sono stati svolti osservando gli uccelli in arrivo mediante l'impiego di binocolo $10 \mathrm{X}$ e di cannocchiale fino a $60 \mathrm{X}$.

Complessivamente sono state rilevate 15 specie di rapaci diurni migratori, delle quali 10 di interesse comunitario e 7 classificate come SPEC - Species of European Conservation Concern secondo BirdLife International (2004), di seguito elencate in Tab. 8.

In totale sono stati censiti 234 individui, dei quali 135 nel 2013 e 99 nel 2014. Le specie di interesse comunitario costituiscono complessivamente il $43,6 \%$ del flusso migratorio. Le specie maggiormente rilevate sono state falco pecchiaiolo, gheppio e poiana, per una percentuale complessiva del 52,6\% rispetto al totale degli individui osservati.

L'aspetto di maggiore interesse emerso consiste nell'elevata diversità di specie rilevate (15). A scopo di raffronto, nel sito di Cima Comér, che presenta una situazione analoga a quella della linea migratoria del Verbano in quanto collocato lungo la sponda occidentale del Lago di Garda, i dati relativi alla migrazione prenuziale indicano che nel 2013 sono state rilevate 14 specie su un totale di 1.468 rapaci censiti e nel 201416 specie su 2.523 rapaci, a fronte di uno sforzo di campo decisamente maggiore rispetto al presente lavoro (Leo \& Micheli, 2013; 2014). Sono state complessivamente dedicate 10 giornate di campo (4 nel 2013 e 6 nel 2014) a tale ricerca e si ritiene che sia opportuno uno sforzo di monitoraggio più intenso e regolare nel tempo, al fine di potere effettuare una valutazione oggettiva dell'importanza di tale flyway non solo dal punto di vista qualitativo, ma anche quantitativo.

\section{MIGRAZIONE AUTUNNALE DEI \\ PASSERIFORMI}

Dalla tarda estate le Alpi sono percorse da un cospicuo flusso migratorio originario delle aree centro nordorientali dell'Europa, un fenomeno ben noto fin dai tempi

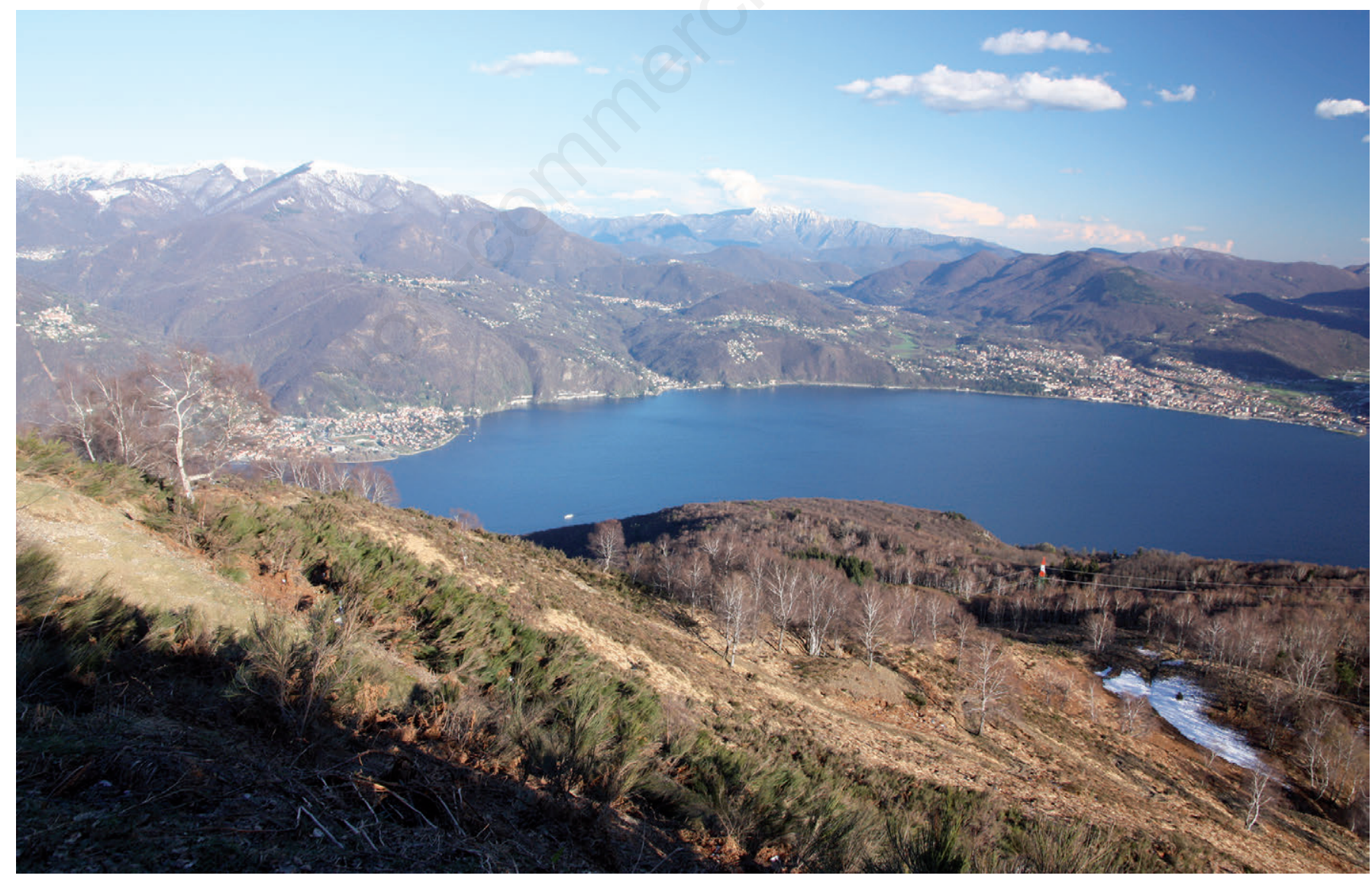

Fig. 32 - Dalla vetta del Monte Carza si domina un ampio tratto di sponda occidentale del Verbano. / The view from Monte Carza dominates a large sector of the Western side of Lake Maggiore. (Foto / Photo Fabio Casale). 
storici e, a partire dagli anni Cinquanta del XX secolo, oggetto di molte ricerche condotte nel settore elvetico dalla Stazione Ornitologica Svizzera di Sempach. Quest'intensa attività di inanellamento, affiancata dall'impiego del radar e, più recentemente, da un'estesa osservazione della migrazione notturna applicando il metodo del moonwatching (Bruderer, 1996; Liechti et al., 1995) ha permesso di delineare le diverse rotte migratorie facendo luce su molti aspetti del comportamento di volo dei migratori in relazione alla morfologia del territorio e al mutare delle condizioni atmosferiche.

Quel che accade sul versante italiano è stato ipotizzato fin dai primi anni Trenta del XX secolo da Antonio Duse (1880-1955), che condusse e coordinò le prime attività di inanellamento sulle Alpi. Secondo i risultati di queste ricerche, il versante meridionale è soprattutto interessato dalla corrente "italo-ispanica" (Duse, 1930), un importante flusso migratorio che da Est si svolge seguendo la fascia prealpina verso Francia meridionale, Baleari e Spagna. Recenti indagini condotte con il moonwatching confermano l'esistenza di questo flusso, ritenuto però meno intenso rispetto a quello rilevato a nord, nelle Alpi svizzere (Liechti et al., 1995).

Lo studio della migrazione sulle Alpi italiane attraverso l'inanellamento è stato progressivamente intensificato a partire dai primi anni Novanta del XX secolo, grazie agli sforzi condotti da un numero sempre maggiore di inanellatori e da gruppi di ornitologi e birdwatcher (Micheli \& Pedrini, 2000). Al fine di rafforzare le conoscenze sulla migrazione post-riproduttiva attraverso le Alpi italiane, l'Istituto Nazionale per la Fauna Selvatica e il Museo Tridentino di Scienze Naturali hanno promosso nel 1997 il "Progetto Alpi", un programma di ricerca pluriennale ideato quale proposta di collaborazione fra gli inanellatori che già operavano o erano intenzionati ad avviare programmi di monitoraggio con tecniche di cattura passiva e in forma standardizzata. Un'analisi dei dati raccolti dal 1997 al 2006 (per un totale di 239.000 esemplari inanellati) porta anzitutto a definire che le prime 10 specie più frequentemente inanellate sono quelle riportate in Tab. 9.

Da tali dati emerge come sia decisamente maggiore la presenza di migratori intrapaleartici rispetto ai migratori transahariani, componente che, salvo poche specie (la più numerosa è la balia nera), sembra sostanzialmente evitare l'attraversamento delle Alpi e mostrare una maggiore abbondanza alle medie e basse quote. L'analisi di dettaglio delle frequenze di cattura all'interno delle diverse stazioni che hanno operato in contemporanea ha permesso inoltre di verificare l'effettiva esistenza della rotta "italo-ispanica" ipotizzata nel passato (Pedrini et al., 2007).

Se la tecnica dell'inanellamento è pratica attualmente piuttosto diffusa al fine di studiare il flusso di migratori attraverso le Alpi italiane, il censimento dei passeriformi

Tab. 8 - Elenco delle specie rilevate durante la migrazione primaverile dei rapaci diurni nel 2013 e 2014, con indicazione dell'inclusione in Allegato I della Direttiva "Uccelli" 79/409/CEE e categoria SPEC e del numero totale di individui censiti. / List of recorded species during spring migration of raptors in 2013 and 2014, with indication of inclusion in Annex I of the Birds Directive 79/409/EEC and SPEC category, and total number of censused individuals.

\begin{tabular}{|c|c|c|c|c|}
\hline Nome scientifico & All. I & SPEC & Numero totale di individui (2013-2014) & $\%$ \\
\hline Pernis apivorus & $\mathrm{X}$ & & 50 & 21,3 \\
\hline Milvus migrans & $\mathrm{X}$ & 3 & 17 & 7,3 \\
\hline Milvus milvus & $\mathrm{X}$ & 2 & 6 & 2,6 \\
\hline Circaetus gallicus & $\mathrm{X}$ & 3 & 8 & 3,4 \\
\hline Circus aeruginosus & 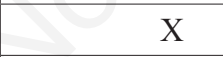 & & 13 & 5,5 \\
\hline Circus cyaneus & +2 & 3 & 1 & 0,4 \\
\hline Circus pygargus & $\mathrm{X}$ & & 2 & 0,8 \\
\hline Accipiter gentilis & & & 18 & 7,7 \\
\hline Accipiter nisus & & & 15 & 6,4 \\
\hline Accipiter sp. & & & 3 & 1,3 \\
\hline Buteo buteo & & & 34 & 14,5 \\
\hline Aquila pennata & $\mathrm{X}$ & 3 & 2 & 0,8 \\
\hline Pandion haliaetus & $\mathrm{X}$ & 3 & 2 & 0,8 \\
\hline Falco tinnunculus & & 3 & 39 & 16,7 \\
\hline Falco subbuteo & & & 12 & 5,1 \\
\hline Falco peregrinus & $\mathrm{X}$ & & 1 & 0,4 \\
\hline Falco sp. & & & 2 & 0,8 \\
\hline Rapace non determinato & & & 9 & 3,8 \\
\hline Totale & 10 & 7 & 234 & 100 \\
\hline
\end{tabular}


migratori mediante l'osservazione diretta ed il contemporaneo conteggio, che permette di dare valutazioni più precise in termini di dimensioni del flusso migratorio e di importanza di alcuni valichi come bottle-neck per i passeriformi, è una pratica ancora poco diffusa in Italia. Si è a conoscenza di un solo progetto che si occupi di individuazione delle rotte e successivo censimento a vista (visual count) dei passeriformi in migrazione attraverso l'arco alpino, quello che si svolge nelle Prealpi Trevigiane (Mezzavilla et al., 2013). Il censimento svolto in due valichi prealpini nel triennio 2004-2006, con uno sforzo di 12 giornate di campo (dall'alba alle h 12-13) per valico per anno, ha permesso di censire numeri complessivi annuali che variano da 6.000 a 20.000 individui, con forti variazioni negli anni, verosimilmente come conseguenza delle condizioni meteorologiche. La famiglia dei fringillidi è risultata essere in assoluto la più abbondante, con valori compresi tra il 78\% e il 93\% (Mezzavilla et al., 2013).

Nel corso del2013 e 2014 (dal22/08/2013 al30/10/2013 e dal 25/08/2014 al 7/11/2014) è stato condotto uno studio preliminare sulla migrazione autunnale dei passeriformi nel Parco Nazionale della Val Grande. L'attenzione è stata focalizzata sui principali valichi alpini presenti nel Parco. Sono stati individuati due valichi interessati da significativi flussi migratori di passeriformi in autunno: Scaredi e Colma di Premosello (Figg. 33 e 34). Altri due valichi indagati (Bocchetta di Vald e Bocchetta di Cavalla) hanno evidenziato flussi migratori meno significativi. I valichi indagati vengono individuati in Fig. 35.

Durante l'attività di monitoraggio dei valichi, sono state complessivamente rilevate 30 specie di passeriformi migratori, elencate in Tab. 10.

In totale sono stati censiti 1.378 individui, dei quali 334 nel 2013 e 1.044 nel 2014. Da tali dati preliminari emerge come il flusso migratorio che interessa i valichi alpini del Parco e rilevabile tramite osservazione diretta nelle ore diurne sia costituito soprattutto da fringillidi (46,9\%, confermando quanto emerso da Mezzavilla et al., 2013), irundinidi $(32,8 \%)$ e motacillidi $(15,6 \%)$, che

Tab. 9 - Specie inanellate con maggior frequenza nell'ambito del "Progetto Alpi" (da: Pedrini et al., 2007). / Most frequently ringed species in the framework of "Progetto Alpi", along Italian Alps (from: Pedrini et al., 2007).

\begin{tabular}{|l|c|}
\hline Specie & Inanellamenti (N) \\
\hline Fringilla coelebs & 45.475 \\
\hline Eritachus rubecola & 31.585 \\
\hline Carduelis spinus & 27.404 \\
\hline Regulus regulus & 15.791 \\
\hline Ficedula hypoleuca & 15.106 \\
\hline Periparus ater & 12.807 \\
\hline Phylloscopus collybita & 8.048 \\
\hline Fringilla montifringilla & 7.592 \\
\hline Sylvia atricapilla & 6.035 \\
\hline Coccothraustes coccothraustes & 5.978 \\
\hline
\end{tabular}

complessivamente rappresentano il 95,3\% degli individui osservati.

Nel 2013 e 2014 sono state dedicate 10 giornate ( 3 nel 2013 e 7 nel 2014) a tale ricerca (Fig. 36). Analogamente alla migrazione dei rapaci diurni, si ritiene che per tale linea di migrazione sia opportuno uno sforzo di campo più intenso e regolare nel tempo, al fine di potere effettuare una valutazione oggettiva dell'importanza di tale flyway.

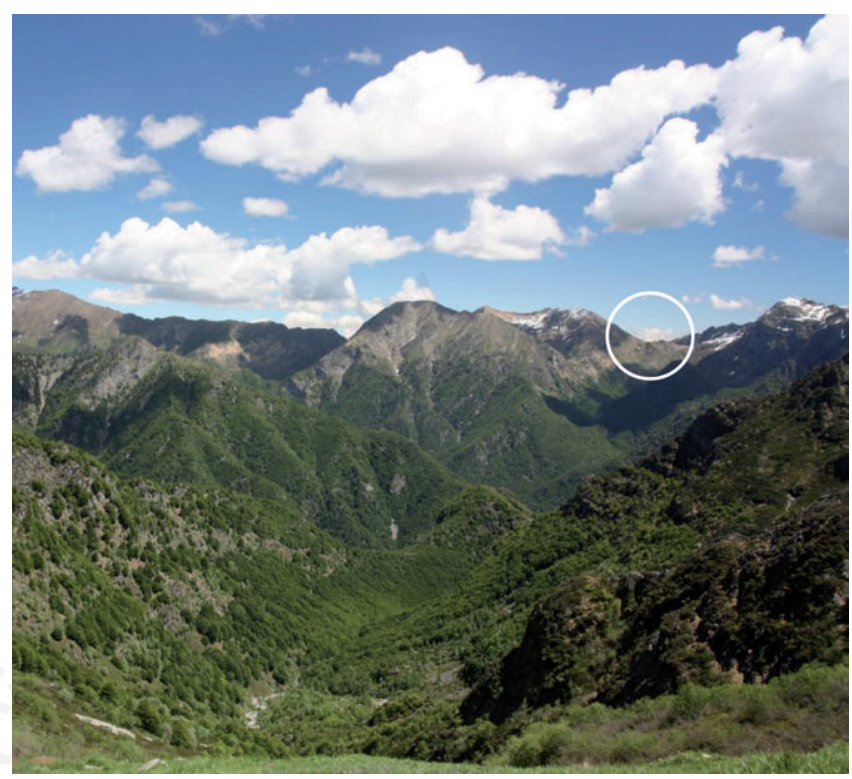

Fig. 33 - La Val Grande dalla Colma di Premosello. Sull'altro lato, verso $\mathrm{N}$, viene evidenziata la località Scaredi. Il flusso migratorio autunnale dei Passeriformi, proveniente da Scaredi, risale la valle e svalica nei pressi della Colma di Premosello, discendendo poi verso S e la valle del fiume Toce. / Val Grande from Colma di Premosello. On the other side, towards $\mathrm{N}$, it is highlighted the position of Scaredi.The autumn migratory route of Passerines comes from Scaredi, follow the valley and passes through Colma di Premosello, than descending towards S and Toce river valley. (Foto / Photo Fabio Casale).

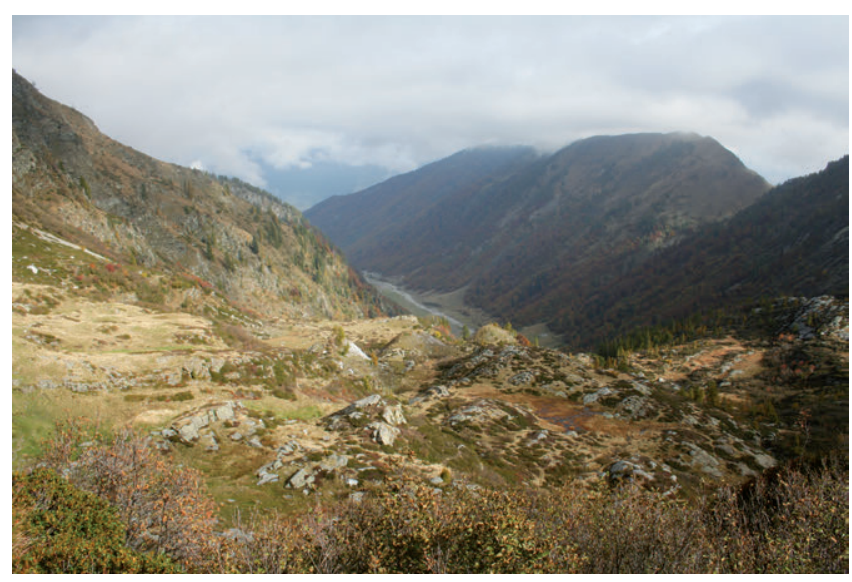

Fig. 34 - La Val Loana da Scaredi. Il flusso migratorio risale la valle a gruppetti o con singoli individui. / Val Loana from Scaredi. The migratory route climb up the valley with small groups or single individuals. (Foto / Photo Fabio Casale). 


\section{DISCUSSIONE E CONCLUSIONI}

Nel Parco Nazionale della Val Grande e aree limitrofe sono state complessivamente rilevate 138 specie di uccelli, ovvero il 35,5\% delle 389 specie note per Piemonte e Valle d'Aosta (Pavia \& Boano, 2009), delle quali 97 nidificanti certe o probabili, ovvero il $46,2 \%$ delle 210 specie nidificanti certe o probabili note per Piemonte e Valle d'Aosta (Pavia $\&$ Boano, 2009) e il 70,3 \% delle 138 specie nidificanti certe o probabili nella provincia del Verbano Cusio Ossola (Bionda \& Bordignon, 2006). I non passeriformi sono 54 $(39,1 \%)$ mentre i passeriformi sono $84(60,9 \%)$.

Di tali specie, 28 sono di interesse comunitario in quanto inserite nell'Allegato I della Direttiva Uccelli 2009/147/ CE (francolino di monte, pernice bianca, fagiano di monte, coturnice, falco pecchiaiolo, nibbio bruno, nibbio reale, gipeto, grifone, biancone, falco di palude, albanella reale, albanella minore, aquila minore, aquila reale, falco pescatore, smeriglio, falco pellegrino, piviere tortolino, gufo reale, civetta nana, civetta capogrosso, succiacapre, picchio nero, tottavilla, calandro, pettazzurro, averla piccola), delle quali una estinta localmente (pernice bianca). Si tratta del 40,6\% delle 69 specie di interesse comunitario note per il Piemonte (Sindaco et al., 2003).

Quarantadue specie (alcune incluse anche nel precedente elenco) risultano altresì essere classificate come di interesse conservazionistico in Europa (SPEC Species of European Conservation Concern), delle quali 11 sono classificate SPEC 2 e 31 come SPEC 3 (BirdLife International, 2004).

A oltre 20 anni dall'istituzione del Parco Nazionale, si segnala quanto segue relativamente ad alcune specie nidificanti di interesse conservazionistico:

- specie tendenzialmente stabili o in aumento nella presenza e/o nella consistenza in periodo riproduttivo: fagiano di monte, falco pecchiaiolo, nibbio bruno, aquila reale, gheppio, falco pellegrino, succiacapre, codirosso comune, pigliamosche, cincia dal ciuffo, cincia bigia, zigolo muciatto;

- specie in declino: coturnice, gufo reale, allodola, culbianco, stiaccino, averla piccola, fanello;

- specie che hanno colonizzato recentemente l'area come nidificanti: francolino di monte, biancone, civetta capogrosso, picchio nero.

Si ritiene che future attività di ricerca e monitoraggio ornitologico debbano essere dedicate a:

- specie di interesse comunitario nidificanti nell'area, al fine di soddisfare le esigenze di regolare monitoraggio di tali specie previste dalla

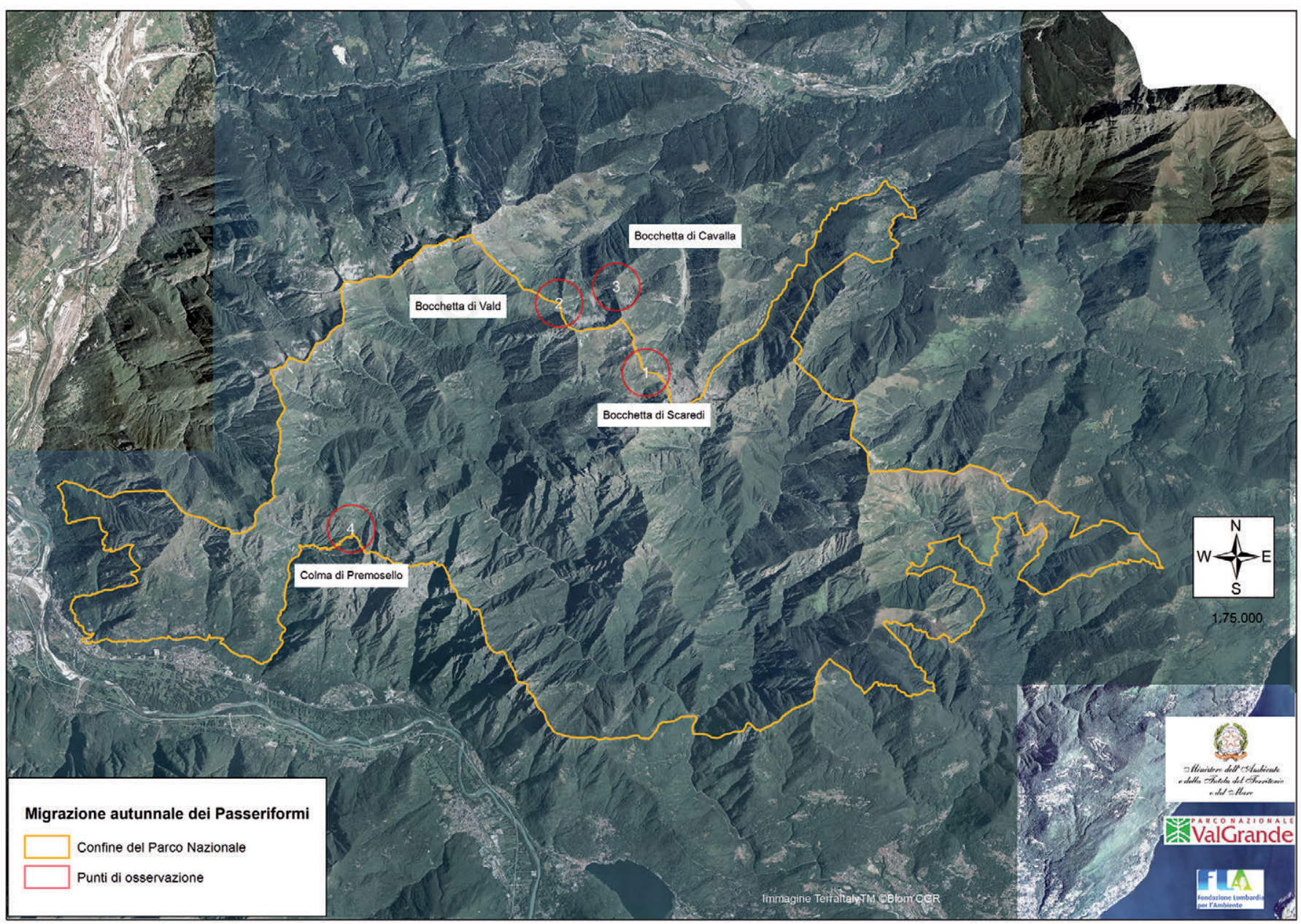

Fig. 35 - Valichi montani interessati dalla migrazione autunnale dei passeriformi. / Mountain passes interested by autumn migration of Passerines. 
Direttiva Habitat: francolino di monte, fagiano di monte, coturnice, falco pecchiaiolo, nibbio bruno, biancone, aquila reale, falco pellegrino, gufo reale, civetta capogrosso, succiacapre, picchio nero, averla piccola;

- specie non di interesse comunitario ma classificate come SPEC o in declino a scala continentale e/o locale: gheppio, allodola, culbianco, stiaccino, codirossone, passero solitario, luì bianco, luì verde, fanello;

- monitoraggio della migrazione primaverile dei rapaci diurni;

- monitoraggio della migrazione autunnale dei passeriformi.

\section{Ringraziamenti}

Rivolgiamo in primo luogo un sentito ringraziamento al Direttore dell'Ente Parco Nazionale della Val Grande, Tullio Bagnati, per la lungimiranza, la passione e la professionalità con cui ha creato le migliori condizioni per svolgere attività di ricerca e monitoraggio dell'avifauna nell'area protetta.

Grazie a Giuseppe Bogliani dell'Università degli Studi di Pavia nonché Direttore della Rivista Italiana di Ornitologia - Research in Ornithology, per avere accolto con entusiasmo la nostra proposta di scegliere la più storica delle riviste italiane di ornitologia per far conoscere alla comunità scientifica i risultati di alcuni anni di attività di ricerca in questo angolo delle Alpi.

Grazie al personale dell'Ente Parco Nazionale della Val Grande, in particolare a Maria Teresa Ciapparella, Fabio Copiatti, Claudio Venturini Delsolaro, Massimo Colombo, Fabio Giovanella e Massimo Scanzio, per

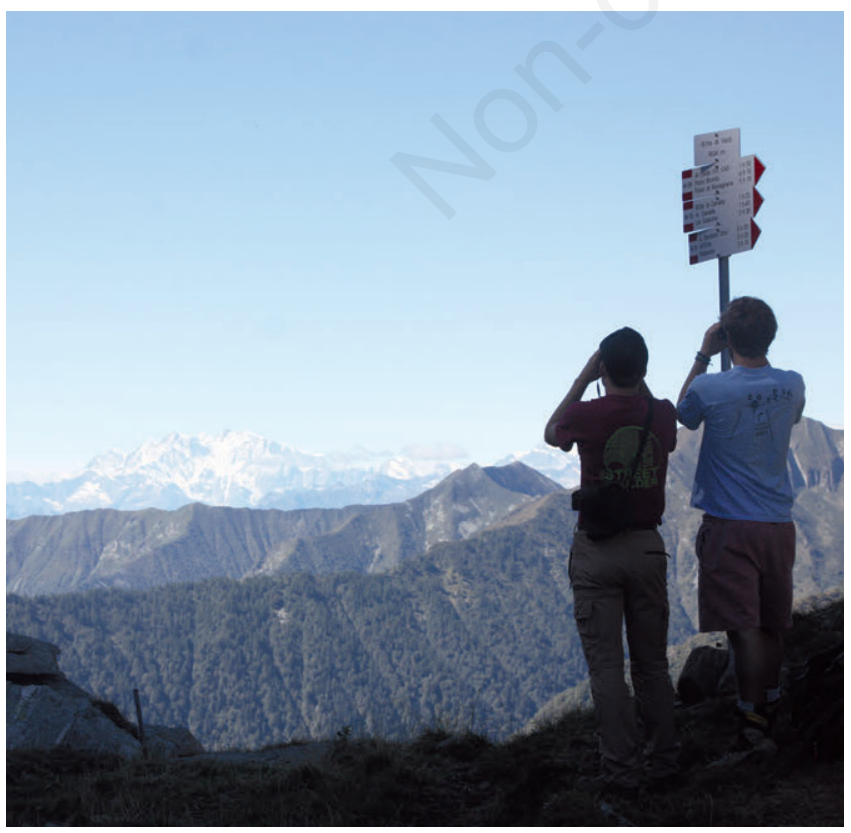

Fig. 36 - Attività di monitoraggio alla Bocchetta di Vald. / Monitoring activity at Bocchetta di Vald. (Foto / Photo Fabio Casale). avere contribuito in vario modo al buon svolgimento delle attività di ricerca e di monitoraggio ornitologico.

Grazie a tutto il personale del CTA (Coordinamento Territoriale per l'Ambiente) del Corpo Forestale dello Stato del Parco, in particolare a Fabio Canepuccia, Pier Francesco Donzelli, Marco Dresco, Eugenio Galbiati, Diego Ramoni e Simone Torniai per avere condiviso con noi le attività di ricerca e il loro entusiasmo per la tutela di un territorio unico quale è quello della Val Grande,

Tab. 10 - Elenco delle specie e numero complessivo di individui rilevati nei punti di monitoraggio della migrazione autunnale dei Passeriformi nel 2013-2014. / List of species and total number of individuals censused through visual counts of passerines during autumn migration in alpine passes in 2013 and 2014.

\begin{tabular}{|l|c|c|}
\hline Nome scientifico & Totale individui & $\mathbf{\%}$ \\
\hline Lullula arborea & 1 & 0,1 \\
\hline Alauda arvensis & 4 & 0,3 \\
\hline Ptyonoprogne rupestris & 13 & 0,9 \\
\hline Hirundo rustica & 5 & 0,4 \\
\hline Delichon urbicum & 434 & 31,5 \\
\hline Anthus campestris & 1 & 0,1 \\
\hline Anthus trivialis & 16 & 1,2 \\
\hline Anthus pratensis & 129 & 9,4 \\
\hline Anthus spinoletta & 59 & 4,3 \\
\hline Anthus sp. & 2 & 0,1 \\
\hline Motacilla flava & 1 & 0,1 \\
\hline Motacilla alba & 5 & 0,4 \\
\hline Turdus viscivorus & 2 & 0,1 \\
\hline Phylloscopus trochilus & 1 & 0,1 \\
\hline Regulus regulus & 2 & 0,1 \\
\hline Cyanistes caeruleus & 8 & 0,6 \\
\hline Parus major & 1 & 0,1 \\
\hline Periparus ater & 37 & 2,7 \\
\hline Fringilla coelebs & 357 & 25,8 \\
\hline Fringilla montifringilla & 1 & 0,1 \\
\hline Serinus serinus & 12 & 0,9 \\
\hline Carduelis carduelis & 35 & 2,5 \\
\hline Carduelis citrinella & 5 & 0,4 \\
\hline Carduelis spinus & 153 & 11,1 \\
\hline Carduelis cannabina & $\mathbf{1 . 3 7 8}$ & $\mathbf{1 0 0}$ \\
\hline Carduelis flammea & & 1,9 \\
\hline Loxia curvirostra & 35 & 0,2 \\
\hline Pyrrhula pyrrhula & & 1,4 \\
\hline Coccothraustes coccothraustes & 0,1 \\
\hline Emberiza citrinella & 26,5 \\
\hline Emberiza cia & 3 & 0,4 \\
\hline Totale & 1 & 0,2 \\
\hline
\end{tabular}


e ai Coordinatori del CFS/CTA Massimo Mattioli fino a giugno 2012 e Andrea Baldi da luglio 2012, per la disponibilità dimostrata e la proficua collaborazione alle ricerche ornitologiche.

Grazie al personale e ai ricercatori della Fondazione Lombardia per l'Ambiente, in particolare a Riccardo Falco, per l'attività di coordinamento dei progetti di ricerca e per la realizzazione delle mappe di distribuzione delle specie, e a Mattia Brambilla, per avere collaborato alla raccolta ed analisi dei dati.

Grazie a due anonimi referee che hanno rivisto e corretto una precedente versione del manoscritto e fornito preziosi suggerimenti per il miglioramento dei suoi contenuti.

Grazie a tutti coloro che in vario modo hanno fornito dati e informazioni utili alla redazione del presente lavoro, anche attraverso la piattaforma www.ornitho.it, in particolare: Elena Auci, Michele Bove, Maria Grazia Carpi, Costante Cavallaro, Fabrizio Clemente, Andrew Cottam, Elena Della Valentina, Piero Dosso, Mercedes Filippi, Diego Fontaneto, Aldo Genoni, Luca Giraudo, Enrico Giussani, Luca Giussani, Jean-Marc Lance, Danilo Manfrin, Raffaele Marini, Giovanni Mastrobuoni, Walter Meneghin, Ian Mifsud, Roberto Molinari, Giovanni Natale, Giuseppe Niccolini, Marta Petrizzi, Pietro Pisano, Lucia Pompilio, Aldo Poppi, Fabrizio Sergio, Giulio Silvestrini, Massimo Soldarini, Fabio Sperotto, Claudia Susanetto, Gianluigi Susanetto, Giuliano Tallone.

Grazie ai fotografi Raffaele Marini e Antonello Turri per avere gentilmente messo a disposizione immagini di avifauna da loro realizzate.

Grazie al Ministero dell'ambiente e della tutela del territorio e del mare e a Fondazione Cariplo per avere concesso i contributi finanziari necessari allo svolgimento delle ricerche ornitologiche.

\section{BIBLIOGRAFIA}

Antoniazza S., 2013 - A' la recherche de la Chouette de Tengmalm Aegolius funereus et de la Chevechette d'Europe Glaucidium passerinum. Nos Oiseaux, 60: 237-240.

Bazzetta G., 1893 - Osservazioni intorno agli Uccelli Ossolani. Annali della Regia Accademia d'Agricoltura di Torino, 36: 127-172.

Bionda R., 2003a - Censimento di Aquila reale Aquila chrysaetos nella provincia del Verbano Cusio Ossola. Avocetta, 23: 33.

Bionda R., 2003b - Censimento di Gufo reale Bubo bubo nella provincia del Verbano Cusio Ossola. Avocetta, 23: 33 .

Bionda R., 2003c - Studio e Piano d'Azione per Falco pellegrino Falco peregrinus nel pSIC e ZPS "Greto del torrente Toce tra Domodossola e Villadossola" e aree limitrofe. Progetto LIFE Natura LIFE02NAT/IT/8572 "Fiume Toce: conservazione di ambienti ripariali a favore dell'avifauna nidificante e migratoria". Provincia del VCO, Verbania. Relazione tecnica non pubblicata.

Bionda R., 2005 - Relazione annuale dell'attività di monitoraggio delle specie di interesse comunitario nidificanti e migratrici nel pSIC e ZPS "Greto del torrente Toce tra Domodossola e Villadossola" e aree limitrofe. Progetto LIFE Natura LIFE02NAT/IT/8572 "Fiume Toce: conservazione di ambienti ripariali a favore dell'avifauna nidificante e migratoria". Provincia del VCO, Verbania.

Bionda R. \& Bordignon L., 2006 - Atlante degli uccelli nidificanti nel Verbano Cusio Ossola. Quaderni di Natura e Paesaggio del Verbano Cusio Ossola, 6. Provincia del VCO, Verbania.

Bionda R. \& Brambilla M., 2012 - Rainfall and landscape features affect productivity in an alpine population of Eagle Owl Bubo bubo. Journal of Ornithology, 153: 167-171.

BirdLife International, 2004 - Birds in the European Union: a status assessment. Wageningen: BirdLife International.

Bocca M. \& Maffei G., 1997 - Gli uccelli della Val d'Aosta. Regione autonoma Valle d'Aosta, Assessorato agricoltura e Foreste; Aosta: Tipografia La Vallée.

Borgo A., 1999 - Influenza della vicinanza di allocco Strix aluco sull'attività di canto territoriale di civetta capogrosso Aegolius funereus. Avocetta, 23: 95.

Borgo A. \& Genero F., 2011 - Monitoraggio Rapaci notturni e Picchio cenerino. Risultati 2010. Regione Veneto. Relazione tecnica non pubblicata.

Bovio F., 1994 - Le migrazioni nella Valle del Ticino. Oleggio: Ente di Gestione del Parco Naturale della Valle del Ticino.

Brembilla R., 2012. Il Pettazzurro, Luscinia svecica svecica, ritorna a nidificare sulle Alpi lombarde. Rivista italiana di Ornitologia, 80 (2): 113-114.

Brichetti P. \& Fracasso G., 2003-2011 - Ornitologia Italiana Voll. 1-7. Bologna: Alberto Perdisa Editore.

Bruderer B., 1996 - Vogelzugforschung im Bereich der Alpen 1980-1995. Ornithologische Beobachter, 93: 119-130. 
Bruderer B. \& Jenni L., 1990 - Migration across the Alps. In: E. Gwinner (ed.), Bird migration: Physiology and ecophysiology. Springer-Verlag, Berlin: 60-77.

Calvi G., Buvoli L., Tonetti J. \& Bonazzi P., $2011-L a$ migrazione degli uccelli nella Valle del Ticino. Dieci anni di inanellamento. Magenta: Parco Lombardo della Valle del Ticino.

Casale F., 2014 - Monitoraggio dell'avifauna del Parco Nazionale della Val Grande. Anno 2013. Ente Parco Nazionale della Val Grande e Fondazione Lombardia per l'Ambiente. Relazione tecnica non pubblicata.

Casale F. \& Brambilla M., 2010a - Fauna di interesse comunitario del Parco Nazionale della Val Grande e del Sito Natura 2000 'Val Grande'. Ente Parco Nazionale della Val Grande e Fondazione Lombardia per l'Ambiente. Relazione tecnica non pubblicata.

Casale F. \& Brambilla M., 2010b - L'avifauna nidificante negli ambienti aperti del Parco Nazionale della Val Grande. Ente Parco Nazionale della Val Grande e Fondazione Lombardia per l'Ambiente. Relazione tecnica non pubblicata.

Casale F. \& Toninelli V., 2005 - La conservazione della biodiversità nelle Alpi occidentali. Quaderni di Natura e Paesaggio del Verbano Cusio Ossola, 5. Provincia del VCO, Verbania.

Casale F., Sala D. \& Bellani A. (a cura di), 2014 - Il patrimonio faunistico del Parco del Ticino negli anni 2000. Magenta: Parco Lombardo della Valle del Ticino e Fondazione Lombardia per l'Ambiente.

De Franceschi P., 1995 - Strategie di gestione dei tetraonidi sulle Alpi italiane: il fagiano di monte (Tetrao tetrix). Supplemento alle ricerche di Biologia della Selvaggina XXII.

De Franceschi C., 2009 - Censimento Gallo forcello nel Parco Nazionale della Val Grande. Ente Parco Nazionale Val Grande. Relazione tecnica non pubblicata.

del Hoyo J., Elliott A., Sargatal J., 1994 - Handbook of the Birds of the World, vol. 2: New World Vultures to Guineafowl. Barcellona: Lynx Edicions.

Dell'Angelo G.B., 1887 - Collezione Ornitologica Vigezzina. Intra: Tip. Bortolotti.

Ente Parchi e riserve naturali del Lago Maggiore, 2007 Il Centro Studi sulle migrazioni nella Riserva Naturale del Fondotoce. Regione Piemonte.

EPNVG - Ente Parco Nazionale Val Grande, 1998 Studi preliminari e redazione delle proposte tecniche del Piano del Parco (art. 12 legge 394/91) e del Regolamento (art. 11 legge 394/91) - Volume II parte terza Fauna. Ente Parco Nazionale della Val Grande. Agriconsulting, Roma.

Fonio E., 1995 - Aspetti naturalistici - La fauna. In: AA.VV., 1995. Val Grande. Storia di una foresta. Anzola d'Ossola: Fondazione Enrico Monti.

Fornasari L. (a cura di), 2003 - La migrazione degli uccelli nella Valle del Ticino e l'impatto di Malpensa. Magenta: Consorzio Parco Valle del Ticino.

Fracasso G., Baccetti N. \& Serra L., 2009 - La lista CISO-COI degli Uccelli italiani - Parte prima: liste A, B e C. Avocetta, 33: 5-24.
Giraudo L., 2007 - La migrazione dei rapaci in Italia. In: Osservatorio regionale sulla fauna selvatica. Le autostrade del cielo: rotte di migrazione dell'avifauna attraverso le Alpi. Atti del convegno. Torino: Regione Piemonte.

Giraudo L. \& Toffoli R., 2003 - La migrazione postnuziale del nibbio bruno Milvus migrans attraverso le Alpi Marittime. Avocetta, 27: 60-62.

G.P.S.O., 1990 - Resoconto ornitologico per la regione Piemonte - Valle d'Aosta. Anni 1988-1989. Rivista piemontese di Storia Naturale, 11: 215-237.

G.P.S.O., 2007 - Resoconto ornitologico per la regione Piemonte - Valle d'Aosta. Anno 2005. Rivista piemontese di Storia Naturale, 28: 383-426.

Horch P. \& Spaar R., 2010 - Lo Stiaccino Saxicola rubetra specie target per l'agricoltura estensiva. Ficedula, 45: 20-27.

Laddaga L. \& Casale F. 2014 - Avifauna della Riserva Naturale Regionale e Oasi LIPU Palude Brabbia. Rivista piemontese di Storia Naturale, 35: 319-366.

Leo R. \& Micheli A., 2013 - La migrazione prenuziale dei rapaci a Cima Comér - Parco Regionale Alto Garda Bresciano (BS). In: Infomigrans, 31: 8.

Leo R. \& Micheli A., 2014 - La migrazione prenuziale dei rapaci a Cima Comér - Parco Regionale Alto Garda Bresciano (BS). In: Infomigrans, 33: 6.

Liechti F., Bruderer B., Parroth H., 1995 - Quantification of nocturnal bird migration by moonwatching: comparison with radar and infrared observations. Journal of Field Ornithology, 66: 457-652.

Micheli A. \& Pedrini P., 2000 - Prime ipotesi sulle rotte migratorie degli Uccelli nel Trentino. Studi Trentini di Scienze Naturali, Acta Biologica, 74.

Mingozzi T., Boano G. \& Pulcher C. (a cura di), 1988 - Atlante degli uccelli nidificanti in Piemonte e Valle d'Aosta. Monografie VII - Museo regionale di Scienze Naturali di Torino.

MovalliC.\& Grimaldi P., 1996-Analisi quali-quantitativa dell'avifauna nidificante nel Parco Nazionale della Val Grande (Piemonte). Rivista piemontese di Storia Naturale, 17: 189-204.

Pavia M. \& Boano G., 2009 - Check-List degli uccelli del Piemonte e della Valle d'Aosta aggiornata al dicembre 2008. Rivista italiana di Ornitologia, 79:23-47.

Pedrini P., Rizzolli F., Rossi F. \& Spina F., 2007 - Il Progetto Alpi: studio della migrazione autunnale degli uccelli sulle Alpi italiane. In: Osservatorio regionale sulla fauna selvatica. Le autostrade del cielo: rotte di migrazione dell'avifauna attraverso le Alpi. Atti del convegno. Torino: Regione Piemonte.

Reteuna D., 1994 - La migrazione degli Accipitriformes, Falconiformes e Ciconiiformes attraverso le Valli di Lanzo. Rivista piemontese di Storia Naturale, 15: 127-153.

Sindaco R., Mondino G.P., Selvaggi A., Ebone A., Della Beffa G., 2003. Guida al riconoscimento di ambienti e specie della Direttiva Habitat in Piemonte. Torino: Regione Piemonte.

Sindaco R., Savoldelli P., Selvaggi A., 2009. La Rete Natura 2000 in Piemonte. I Siti di Importanza Comunitaria. Torino: Regione Piemonte.

Toffoli R. \& Bellone C., 1996 - Osservazioni sulla migrazione autunnale dei rapaci diurni sulle Alpi Marittime. Avocetta, 20: 7-11. 